\title{
City of Manchester State of the Voluntary, Community and Social Enterprise Sector 2017
}

\section{$A$ report on social and economic impact}

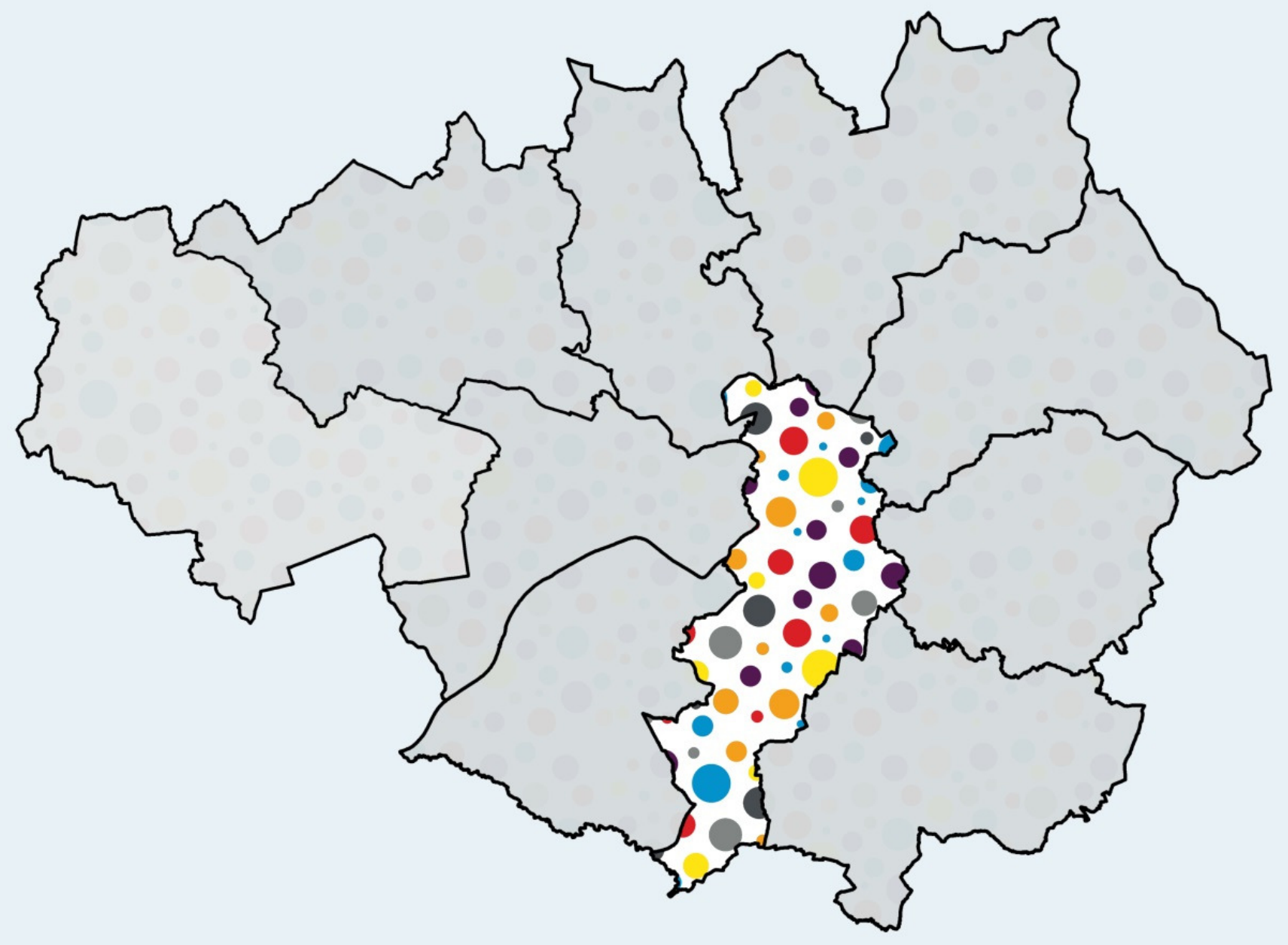

Sheffield Hallam

Centre for

Regional Economic

University and Social Research 


\section{City of Manchester State of the Voluntary, Community and Social Enterprise Sector 2017}

Centre for Regional Economic and Social Research

Sheffield Hallam University

Chris Damm

loannis Prinos

Elizabeth Sanderson

DOI: $10.7190 /$ cresr.2017.8679557866

June 2017 


\section{Acknowledgements}

This research has been commissioned by Macc as part of $10 \mathrm{GM}^{1}$ with GMCVO and undertaken by the Centre for Regional Economic and Social Research (CRESR) at Sheffield Hallam University. This city of Manchester report forms part of a wider collection of reports: there is a Greater Manchester wide report and a report for each of the other local authority areas participating in the study ${ }^{2}$. The full collection of reports can be downloaded from the CRESR website (http://www.shu.ac.uk/research/cresr/reports) and the websites of the project partners.

In completing the report we are particularly grateful to members of the Research Steering Group ${ }^{3}$ and the Macc team for their support in developing and administering the survey and for their input into earlier drafts of the report. We are also grateful to the many employees and volunteers from across the voluntary, community and social enterprise sector who took the time to complete a questionnaire or participate in a focus group.

\section{Contact information}

For CRESR:

$\begin{array}{ll}\text { Name: } & \begin{array}{l}\text { Elizabeth Sanderson } \\ \text { Address: }\end{array} \\ & \text { Research Fellow } \\ & \text { Unit 10 Science Park } \\ & \text { City Campus } \\ & \text { Howard Street } \\ & \text { Sheffield } \\ & \text { S11WB } \\ \text { Tel: } & 01142254814 \\ \text { Email: } & \text { e.sanderson@shu.ac.uk }\end{array}$

For Macc:

$\begin{array}{ll}\text { Name: } & \begin{array}{l}\text { Mike Wild } \\ \text { Chief Executive } \\ \text { Swan Buildings } \\ \text { 20 Swan Street } \\ \text { Manchester }\end{array} \\ & \text { M4 5JW } \\ \text { Tel: } & 01618349823 \\ \text { Email: } & \text { mike@macc.org.uk } \\ \text { Twitter: } & \text { @MikeWildMacc }\end{array}$

\footnotetext{
${ }^{1} 10 \mathrm{GM}$ is a joint venture by the Greater Manchester Voluntary Sector Infrastructure Organisations (Action Together in Oldham and Tameside, Bolton CVS, CVS Rochdale, Macc, Salford CVS, VCAT, Wigan \& Leigh CVS)

${ }^{2}$ The other areas are: Bolton, Salford, Oldham, Rochdale, Stockport, Tameside and Wigan.

${ }^{3}$ The following organisations were represented on the Research Steering Group: CRESR, Salford CVS (lead partner), Greater Manchester Centre for Voluntary Organisation (GMCVO), Bolton CVS, Macc, Action Together in Oldham and Tameside and CVS Rochdale.
} 


\section{Definitions}

This report is about the 'state of the voluntary, community and social enterprise sector in the City of Manchester'. At various times the voluntary sector has been known as the 'voluntary and community sector' or the 'third sector' whilst the current Government talks a lot about 'civil society'. In this report, when we talk about the voluntary sector in the City of Manchester, we mean voluntary organisations, community groups, the community work of faith groups, and those social enterprises where there is a wider accountability to the public via a board of trustees or a membership and all profits will be reinvested in their social purpose. 


\section{Foreword}

In the introduction to the 2013 edition of this report, I said that it had been a long-held ambition of mine to be able to show the full extent of the enormous contribution which voluntary organisations, community groups, social enterprises and the community work of faith groups make to Manchester. In the intervening four years, the key findings from that report have been accepted as the definitive statement of this contribution. Some of the numbers are burned into my memory. We often use them to introduce events and presentations, asking people to guess things like how many volunteers there are in Manchester. The wide-eyed reactions to the scale and wealth of effort happening in communities are always a pleasure to see. It has changed the conversation about this sector with public officials, politicians, businesses and the general public.

So you won't be surprised that not all that much further down my wish list was a desire to repeat the exercise and show the changes in the sector over time: to turn a snapshot into a story. That, for the first time, is what this new report does.

The intervening years have been particularly challenging for those working to deliver services and support to the public as the economic climate has been especially harsh for our organisations and the people they support. And yet this report tells the story of a sector which is working incredibly hard to meet growing challenges. It is a complex picture in which headline increases in some of the key numbers simultaneously represent an enormous wealth of effort while masking changes which reflect those ever-increasing pressures.

This report will show you the latest picture of the real scale of this sector in Manchester. It is a story of local people engaged in volunteering, working alongside a significant paid workforce. It shows the diversity of the organisations and reveals the economy in which they operate. It shows the scope of the activities, services and interventions and the reach both into and out from local communities.

As with the 2013 report, I hope these data will challenge and astonish in equal measure. I hope it will encourage you to see that there is still an enormous (but often unseen) asset in our economy, sometimes called the "social sector", which is playing its part as a vibrant, intricate engine of social good. I hope it will impress upon you the scale and spread of that effort which, despite the pressures of recent years, continues to renew and grow where it is called upon.

I want to thank our 10GM partners (the support and development organisations in neighbouring boroughs in Greater Manchester), GMCVO and Sheffield Hallam University for their collaboration in making this this happen. I particularly want to thank all those organisations who took time to complete the survey for it is their combined efforts which this report ultimately shows.

Above all, I want to thank anyone who reads this report and uses it to consider how they, in whatever capacity they can, chooses to work with local charities, voluntary groups, community organisations and social enterprises to nurture and grow our social economy as a fundamental part of Manchester's success.

Mike Wild

Chief Executive

Macc - Manchester's local voluntary and community sector support organisation.

April 2017 


\section{Contents}

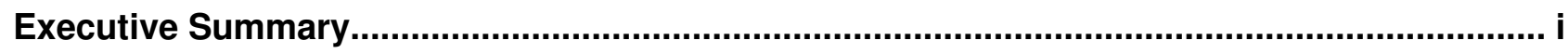

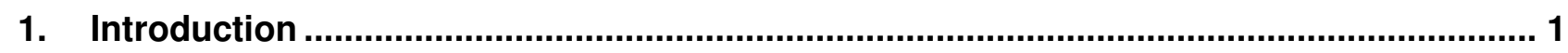

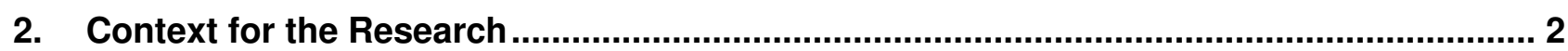

3. What the Voluntary Community and Social Enterprise Sector in the City of Manchester

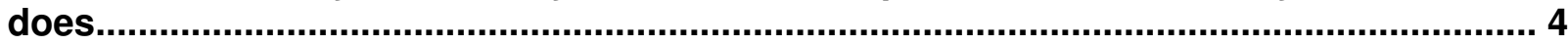

4. Who the Voluntary Community and Social Enterprise Sector in the City of Manchester works with..........................................................................................................................11

5. Finances and Income

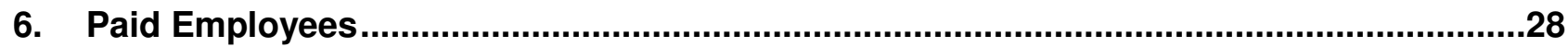

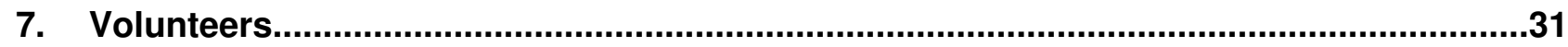

8. Partnership Working: the Public Sector .....................................................................36

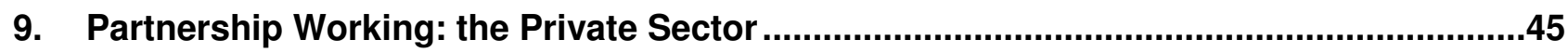

10. Partnership Working: Voluntary Community and Social Enterprise Organisations.........48

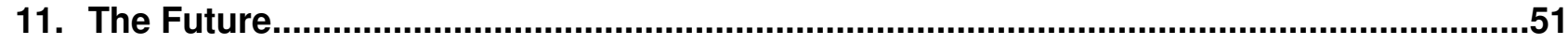

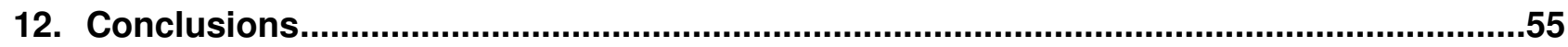

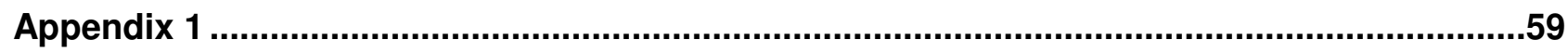




\section{Executive Summary}

This report provides the main findings of research aimed at improving the understanding of the social and economic impact of the voluntary, community and social enterprise (VCSE) sector in the City of Manchester. The key objective of the research was to provide a comprehensive overview of the sector in Manchester at the start of 2017.

In this summary we answer eleven key questions about the sector and its role across the City of Manchester.

\section{Q1. How many organisations are there?}

There are an estimated 3,394 organisations working in the VCSE sector in Manchester and the vast majority of organisations are micro or small (81 per cent less than $£ 100,000)$ :

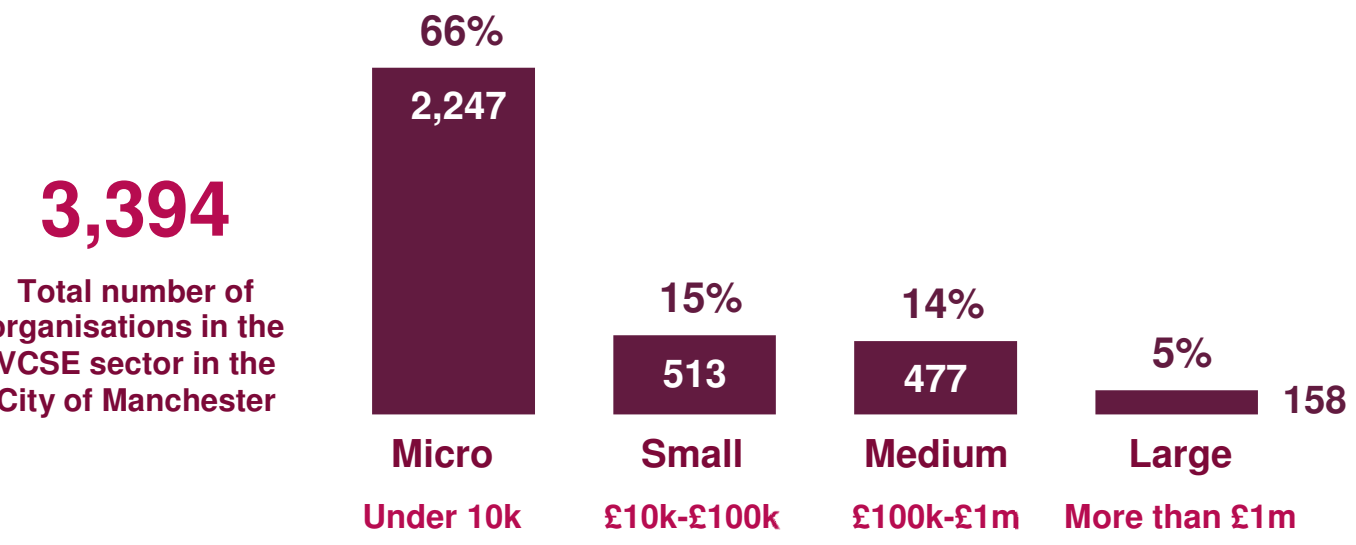

\section{Q2. Who benefits from their work?}

Over one quarter (27 per cent) of organisations surveyed identified 'everyone' as their main clients, users or beneficiaries. A similar proportion of organisations (26 per cent) identified children and young adults as a main client group.
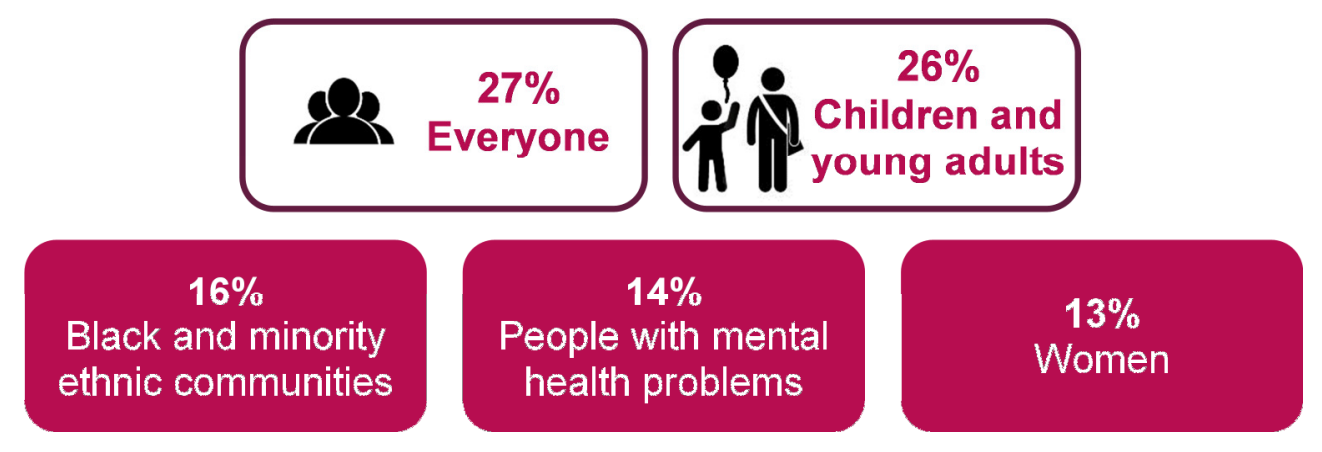

MAIN CLIENT GROUPS IN 2012/13:

EVERYONE - 33\%

YOUNG PEOPLE - 25\%

WOMEN - $23 \%$
OLDER PEOPLE - $21 \%$

BLACK AND MINORITY ETHNIC COMMUNITIES - $20 \%$

CHILDREN - $19 \%$ 
It is estimated that the VCSE sector in the City of Manchester made:

\section{1 million interventions with clients, users or beneficiaries in the past year}

The VCSE sector works at a range of different geographical levels: both across and beyond Manchester the local authority area, and specific communities and neighbourhoods within it, are the main focus for a majority of organisations:
Particular Manchester neighbourhoods and communities

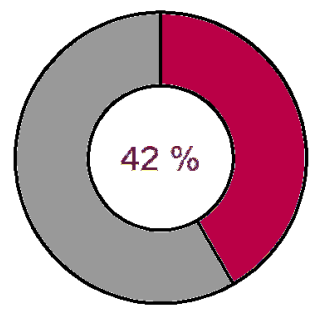

Across the whole Manchester Local Authority area

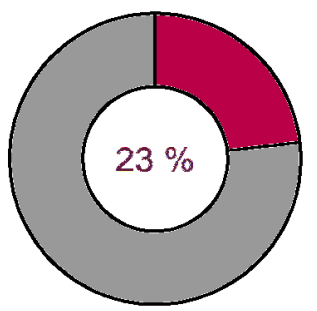

\section{Q3. What does the VCSE sector in the City of Manchester do?}

The areas with the greatest proportion of organisations working in them are:

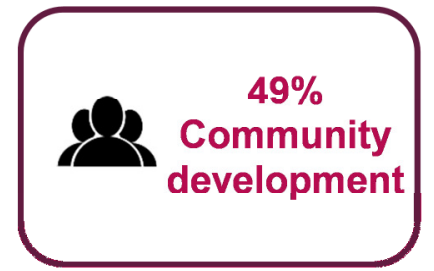

MAIN AREAS IN 2012/13:

COMMUNITY DEVELOPMENT - $43 \%$ HEALTH \& WELLBEING - $40 \%$
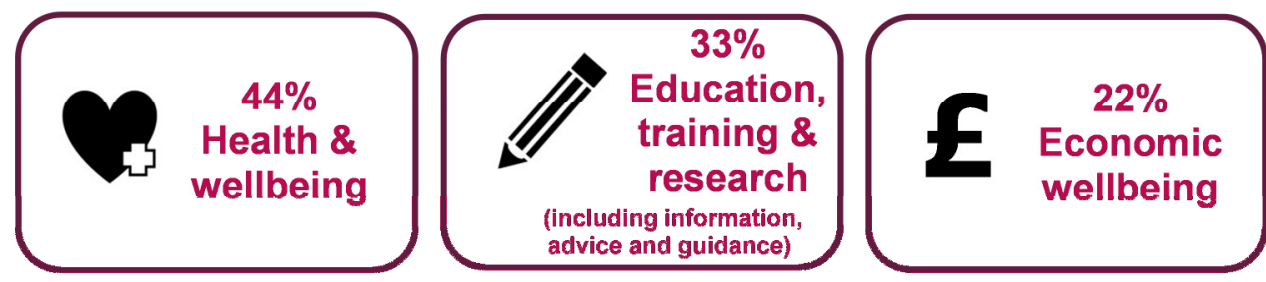

EDUCATION, TRAINING \& RESEARCH - 36\% ARTS, HERITAGE \& CULTURE- $21 \%$

\section{Q4. What is the income of the VCSE sector in the City of Manchester?}

Total income in $2014 / 15$ is estimated to be $£ 526 \mathrm{~m}$, an increase of three per cent compared to 2013/14.

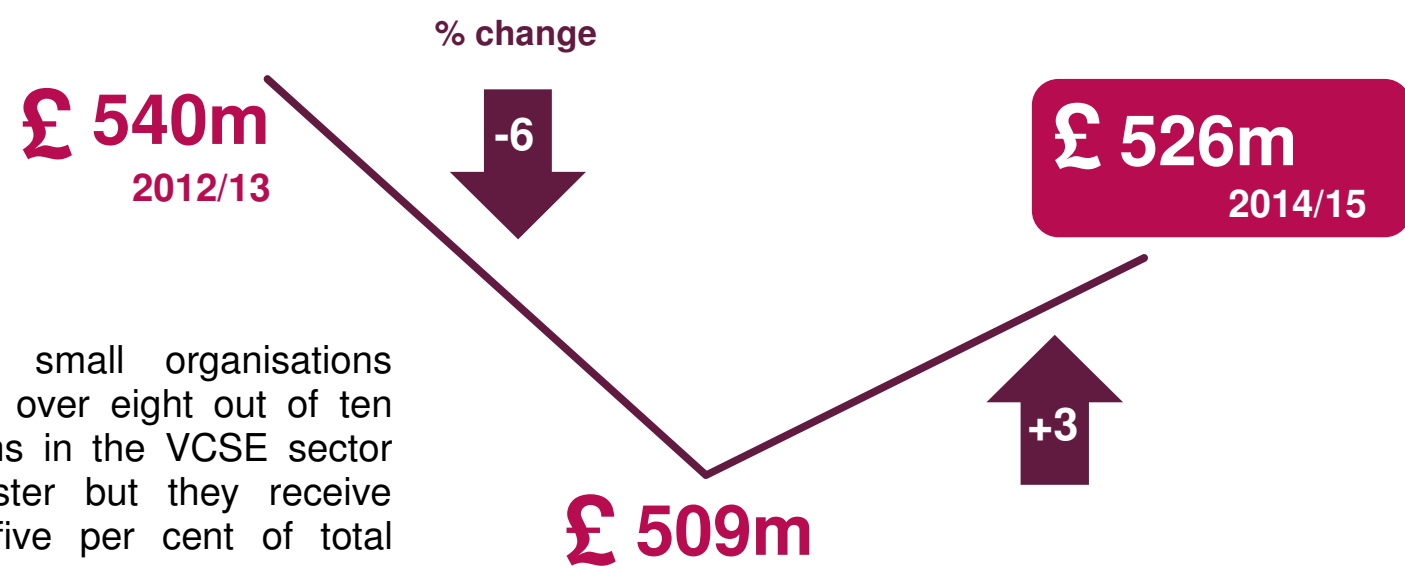

2013/14

Across Greater Manchester micro and small organisations experienced year on year reductions in total income between 2012/13 and 2014/15.

By contrast medium and large organisations saw a reduction in total income between $2012 / 13$ and $2013 / 14$ but then an increase between 2013/14 and 2014/15. But income is still below 2012/13 levels. 
Q5. Where does the VCSE sector in the City of Manchester receive its funding from?

\section{$89 \%$ have at least one source of non-public sector funds \\ $71 \%$ IN $2012 / 13$}

\section{INCLUDING:}

Grants from charitable trusts and foundations (received by 58 per cent of respondents)

Fundraising (52 per cent of respondents)

Grants from National Lottery distributors (45 per cent)

MAIN SOURCES OF FUNDING IN 2012/13:

FUNDRAISING - 29\%

GRANTS FROM CHARITABLE TRUSTS \& FOUNDATIONS - 24\%

CHARGING FOR GOODS \& SERVICES - $18 \%$

\section{$71 \%$ have at least one source of public sector funds \\ $66 \%$ IN 2012/13}

\section{INCLUDING:}

Manchester City Council (received by 52 per cent of respondents)

NHS Manchester Clinical

Commissioning Group (11 per cent)

MAIN SOURCES OF FUNDING IN 2012/13:

MANCHESTER CITY COUNCIL - 36\% NHS MANCHESTER - $7 \%$

\section{Q6. How sustainable is the VCSE sector in the City of Manchester?}

\section{The survey highlights some areas for concern:}

- 57 per cent of respondents reported increasing their expenditure but only 47 per cent had experienced an increase in income

- 31 per cent of respondents reported a decrease in income but only 18 per cent reduced their expenditure

- 35 per cent reported a reduction in their financial reserves.

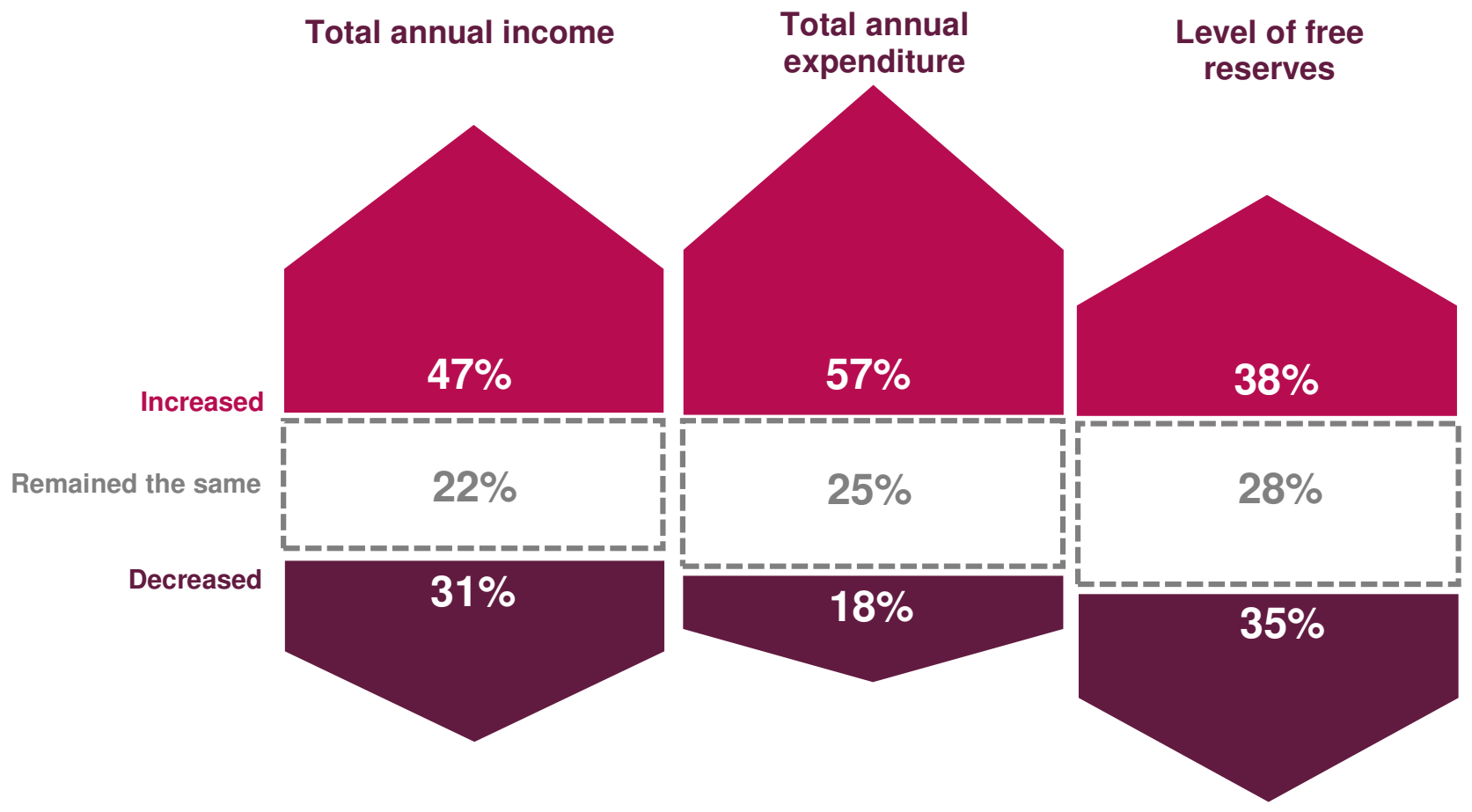


The precarious financial situation of some organisations is further emphasised by the state of their reserves:

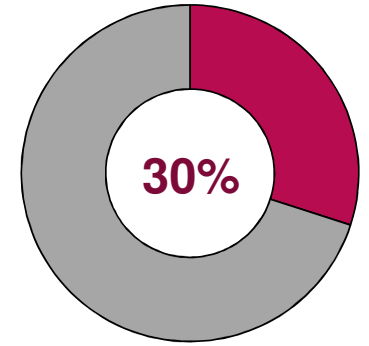

Proportion of organisations with reserves less than one month's expenditure

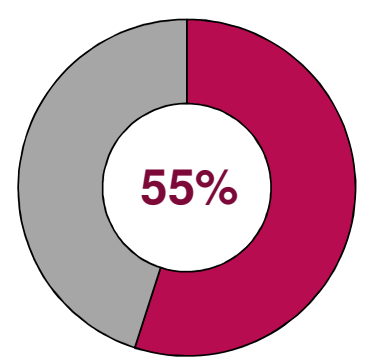

Proportion of organisations with reserves less than

25 per cent of annual expenditure

\section{Q7. Who works and volunteers in the VCSE sector?}

The sector is supported by:

110,900 volunteers $\&$ committeel board members $(87,400$ volunteers and 23,500 committee/board members)

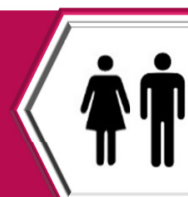

who donated

278,600 hours

of their time per week

$£ 252.1$ million per annum

= estimated economic contribution

of volunteers
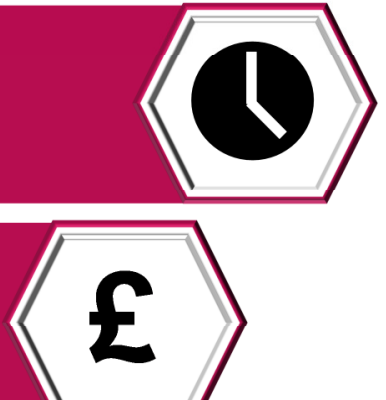

The VCSE sector is also a significant employer. There are an estimated:

\section{1,000 FTE paid staff employed in the VCSE sector in the City of Manchester}

\section{7 million per annum}

contributed to the economy by paid employees of City of

Manchester VCSE sector organisations 


\section{Q8. How good are relationships with public sector bodies?}

Survey respondents had dealings with a range of local public sector bodies:

\section{$84 \%$ had some dealings with Manchester City Council}

\section{$61 \%$ had some dealings with the University of Manchester}

\section{$58 \%$ had some dealings with Greater Manchester Police}

$39 \%$ had some dealings with NHS Manchester Clinical Commissioning Group

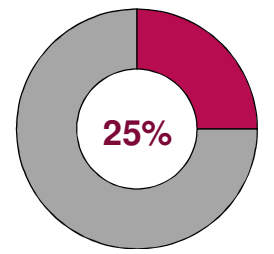

... of VCSE organisations are satisfied with their ability to influence Manchester City Council, lower than the proportion in 2012/13 (30 per cent). More respondents (32 per cent) are satisfied with their ability to influence their most frequent other public sector contact.

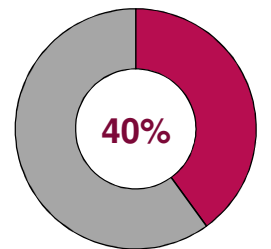

... of VCSE organisations felt Manchester City Council is a positive influence on their success, slightly lower than the proportion in 2012/13 (44 per cent). More respondents (53 per cent) also felt that their most frequent other public sector contact was a positive influence on their success.

\section{Q9. How well does the VCSE sector work with private businesses?}

\section{$65 \%$ had some dealings with local private businesses}

.. 27 per cent of respondents felt that the private business community in Manchester was a positive influence on their organisation's success - very similar to the proportion in $2012 / 13$ of 26 per cent

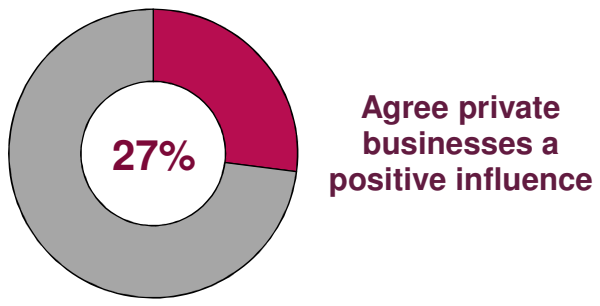

\section{$3 \%$ are members of a private sector-led consortium}

\section{Q10. How well does the VCSE sector work together?}

$76 \%$ had a 'great' or 'fair amount' of contact with other VCSE organisations in the City of Manchester (50 per cent with VCSE organisations in Greater Manchester)
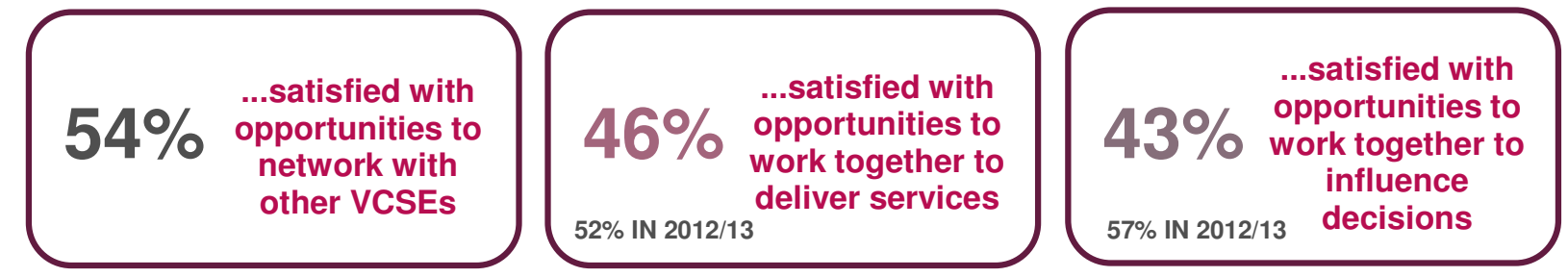

$26 \%$ are members of a formal VCSE consortium 


\section{Q11. What are the key issues facing the VCSE sector in the future?}

Respondents were asked about the strategies they are actively pursuing or planning to pursue. Around seven in ten respondents or more were already doing or planning to do the following:

\section{$79 \%$ work more closely with another voluntary/not-for-profit organisation \\ $71 \%$ increase earned income \\ $69 \%$ change the way they run their services or activities}

Respondents were also asked to consider the factors they anticipated assisting or constraining their organisation over the next 12 months:

\section{Assisting}

\section{TOP FACTORS:}

Ability to secure other sources of income (54 per cent assisting or greatly assisting)

Engagement with other VCSE organisations (52 per cent)

\section{Constraining}

\section{TOP FACTORS:}

The local economy (36 per cent constraining or seriously constraining)

Ability to secure grant funding from public sector bodies (32 per cent) 


\section{Introduction}

This report provides the main findings of research aimed at improving the understanding of the social and economic impact of the voluntary, community and social enterprise (VCSE) sector in the City of Manchester. The research was commissioned by Macc as part of $10 \mathrm{GM}^{4}$ with GMCVO and undertaken by the Centre for Regional Economic and Social Research (CRESR) at Sheffield Hallam University.

The key objective of the research was to provide a comprehensive overview of the sector in the City of Manchester at the start of 2017.

The research involved a web-based survey of organisations supporting the people and communities of the City of Manchester and focus groups with VCSE organisations. The research took place between September 2016 and January 2017.

Appendix 1 provides further detail on the research methodology.

${ }^{4} 10 \mathrm{GM}$ is a joint venture by the Greater Manchester Voluntary Sector Infrastructure Organisations including Salford CVS (lead partner on this research), Action Together in Oldham and Tameside, Bolton CVS, CVS Rochdale, Macc and Wigan and Leigh CVS. 


\section{Context for the Research}

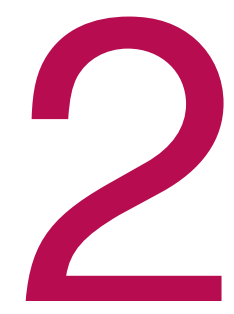

This research comes during both a period of slow economic recovery following the recent long-term economic downturn and a rapidly changing political backdrop as the UK prepares to exit the European Union and the devolution agenda gains pace.

NCVO report that between 2012/13 and 2013/14 the income and spending of the voluntary and community sector in the UK increased, the first notable net growth since the peaks of 2007/08 and 2009/10 respectively. ${ }^{5}$ Total income has increased by just over £2.4bn to £43.8bn and now exceeds the 'peak income' seen in 2007/08 (£43.2bn). NCVO also report that following a decrease in income from government after 2009/10, income from government increased between 2012/13 and 2013/14 by around £0.5bn, although this remains below 2009/10 levels. The majority of this increase was in the largest charities which means the impact might not be felt as keenly at a local level. Income from individuals has also increased by just over £1bn between 2012/13 and 2013/14 and is now at its highest ever level.

While these figures provide reasons to be optimistic there is still need for caution. With the election of the Conservative Government in May 2015, austerity measures are set to continue for the foreseeable future and VCSE organisations are likely to feel the impact of these measures. This includes the Government's commitment to a continuing programme of welfare reform which is likely to result in increasing demand for some services as benefits are restricted or withdrawn. The total anticipated reduction by 2020/21, from both pre and post-2015 welfare reforms in the City of Manchester, is predicted to be $£ 312 \mathrm{~m}$ per year or equivalent to $£ 850$ per working age adult per year. ${ }^{67}$ These reforms are likely to continue to put pressure on VCSE organisations both in terms of their financial health and the need to meet greater levels of need from existing and new beneficiaries.

Locally, the reductions in public expenditure have been felt acutely in the City of Manchester. As part of the Coalition Government's plan to reduce the deficit, it reduced funding for local government in England. Across Greater Manchester local authorities have experienced, and are continuing to experience, a decline in Government funding.

\footnotetext{
${ }^{5}$ UK Civil Society Almanac (2016) NCVO.

${ }^{6}$ Beatty and Fothergill (2016) The Uneven Impact of Welfare Reform: The financial losses to places and people.

${ }^{7}$ Note: These figures are based on HMRC Budgets and Autumn Statements from between 2010 and 2015. In the 2016 Autumn statement the Pay-to-stay measure was scrapped and so this has been taken account of in the figures. The estimate of cuts due to the LHA Cap in social housing was increased by a further $£ 160 \mathrm{~m} \mathrm{p.a.} \mathrm{which}$ is not taken account of in the figures. The Universal Credit Taper was also increased by $2 p$ in the pound, an increase in funding of $£ 570 \mathrm{~m}$ p.a., which is not included in the figures presented here.
} 
Against this background this research provides in depth data about the 'state of the VCSE sector' in the City of Manchester at the start of 2017. The research provides a comprehensive overview of the sector in the City of Manchester for partners to draw upon and help harness the potential of the sector. 


\section{What the Voluntary Community and Social Enterprise Sector in the City of Manchester does}

This chapter develops a picture of the core features of the voluntary, community and social enterprise (VCSE) sector in the City of Manchester. It focuses on a series of general questions in which respondents were asked about their group or organisation: what it is and what it does.

\subsection{How many VCSE organisations are there in the City of Manchester?}

Estimating the number of organisations represents a major challenge. This is because a large proportion of organisations are small, local and not formally constituted as charities, limited companies or other recognised forms which require registration (e.g. industrial and provident societies). As a result they do not appear on formal central records such as those held by the Charity Commission or Companies House so are considered 'below the radar' (BTR). Any estimate of the total number of organisations in an area therefore requires information on the numbers of registered and unregistered (i.e. BTR) organisations.

In estimating the total number of organisations in the City of Manchester we drew on information from the following sources:

- $\quad$ The Register of Charities in England and Wales, which indicated 930 registered charities with postcodes in the City of Manchester.

- The ratio of charities to non-charities provided in the 'National Survey of Charities and Social Enterprises' (NSCSE), undertaken by Ipsos MORI for the Cabinet Office in 2010. This was used to gross the estimate upwards to a total of 1,453 registered organisations, to take account of non-charitable social enterprises.

- $\quad$ Research by NCVO and the University of Southampton ${ }^{8}$ which found that on average there are 3.66 BTR organisations per 1,000 population. If this figure is

\footnotetext{
${ }^{8}$ Mohan, J et al. (2010). Beyond 'flat-earth' maps of the third sector: enhancing our understanding of the contribution of 'below-the-radar' organisations. Northern Rock Foundation Briefing Paper
} 
applied to the City of Manchester ${ }^{9}$, it can be estimated that there are 1,941 BTR organisations in the City. ${ }^{10}$

Summing the estimated numbers for both registered and BTR organisations produces an estimated figure of:

\section{3,394 organisations in total operating in the VCSE sector in the City of Manchester.}

This is higher than the estimate produced for the 2013 report $(3,093)$. Whilst this may reflect a genuine increase in the number of voluntary organisations between the two surveys this could also in part be due to unavoidable differences in the estimation methodology.

For the 2013 report, the sampling frame for the NSCSE was used to provide the estimates for the number of formally registered organisations. Unfortunately this survey was subsequently cancelled and has not been repeated. As such, only the ratio of charities to non-charities was taken from this data source and combined with the number of charities from the charity register.

\subsection{What size are organisations in the City of Manchester?}

The size of organisations is traditionally measured using their annual income ${ }^{11}$. When the distribution of organisations across Greater Manchester was explored by size category based on income for 2014/15, it showed that the majority of organisations were either micro or small. But the survey was underrepresentative of BTR organisations (only 23 per cent of survey respondents were identified as BTR), so this did not present an accurate picture of the actual distribution. The figures were therefore adjusted based on the assumption that the estimated 1,928 organisations not included in the survey sample were BTR and micro in size ${ }^{12}$.

Exploring the distribution by size category based on income for $2014 / 15$ also showed some inconsistency with the distribution found in 2013. Therefore, in order to provide the most robust estimate of the distribution of organisations in the VCSE sector by size, data from both waves of the survey have been used to calculate the proportion of organisations estimated to be in each size category.

The outcome of this process is shown in figure 3.1 , which demonstrates that an estimated 66 per cent of the VCSE sector (2,247 organisations) are micro in size, 15 per cent are small (513 organisations), 14 per cent are medium (477 organisations), and five per cent are large (158 organisations).

Introducing the BTR figure produces a much higher estimate for the number and proportion of micro organisations and emphasises the finding that a large proportion of organisations in the VCSE sector in the City of Manchester are very small (81 per cent micro or small). This is similar to the national picture: $\mathrm{NCVO}^{13}$ estimate that 83 per cent of the VCSE sector is made up of micro or small organisations, 14 per cent

\footnotetext{
${ }^{9}$ Based on Office for National Statistics 2015 population estimates

${ }^{10}$ It is important to note that the BTR figure is an estimate based on an average across 46 local authorities. The BTR research found significant variability, with some local authorities reaching over seven BTR organisations per 1,000 population, and in one case exceeding 10.

${ }^{11}$ In exploring organisation size we used the categories developed by NCVO for use in their Almanac series (see e.g. Clark, J et al., 2010)

${ }^{12}$ The basis for these assumptions is discussed in more detail in the methodological annex

13 UK Civil Society Almanac (2016) NCVO.
} 
are medium, and three per cent are large. Results are also consistent with the pattern across Greater Manchester as whole, where 90 per cent of organisations are micro or small, eight per cent are medium and two per cent are large.

Figure 3.1: Proportion of City of Manchester VCSE organisations by size (estimated)

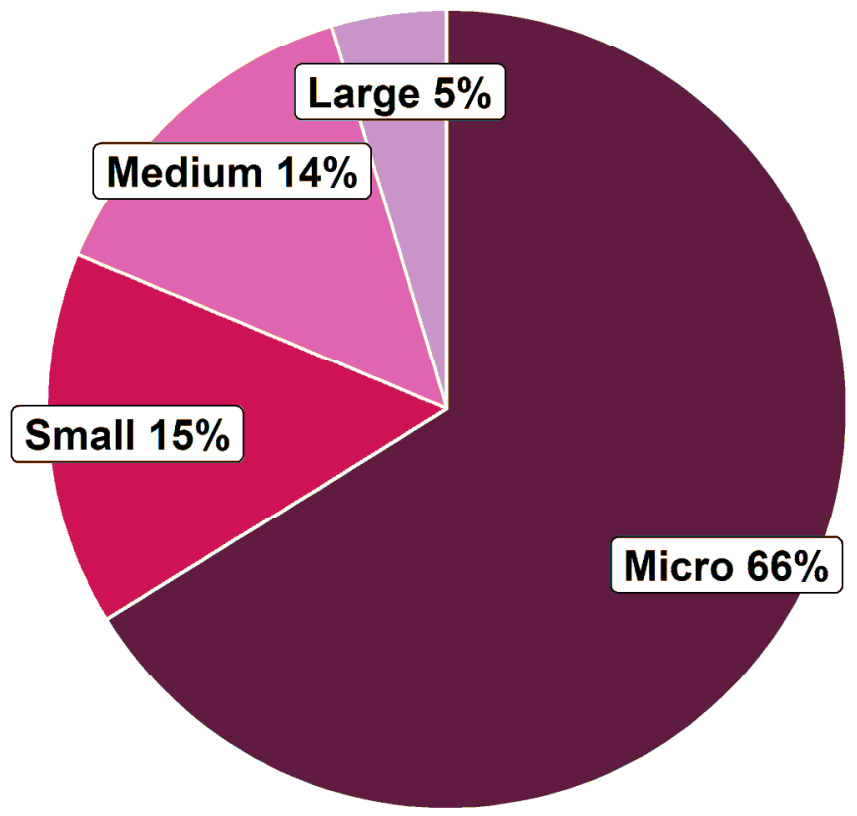

Source: City of Manchester State of the VCSE sector survey 2016/17

Base: 125

\subsection{What types of organisations operate in the VCSE sector in the City of Manchester?}

Respondents to the questionnaire were asked were asked to identify which category from a list of 'organisation types' best described their organisation. The results indicate that many organisations in the VCSE sector are likely to have a local focus. Figure 3.2 shows that the largest proportion, 24 per cent, identified their organisation as being a local voluntary organisation. Notable proportions also identified themselves as: a Greater Manchester city regional VSO (14\%); community or neighbourhood group (12 per cent); and social enterprise (10 per cent).

Other types of organisation were less common: just four per cent of respondents stated they were faith groups; four per cent were a local branch of a national VSO; five per cent were North West regional VSOs; and a further five per cent were sport, leisure or social clubs. Finally, seven per cent identified as a community centre or village hall.

This breakdown of organisations by type followed a similar pattern to that in the 2012/13 survey, where the two largest categories were: local voluntary organisation (24 per cent) and community or neighbourhood group (16 per cent). The proportion identifying as a faith group, however, decreased from eight per cent in 2012/13 to just four per cent in 2016/17. Local branches of national organisations were also more common in 2012/13 (eight per cent; four per cent in 2016/17). Figures for regional organisations are not directly comparable as the category was split into Greater Manchester regional and North West Regional for the 2016/17 survey. 
The analysis across Greater Manchester found a similar picture with local voluntary organisations (22 per cent) and community or neighbourhood groups (15 per cent) accounting for 38 per cent of respondents. Only 13 per cent of respondents stated they were either a national voluntary organisation (two per cent), a branch of a national voluntary organisation (six per cent) or an affiliated member of a national voluntary organisation (four per cent). The percentage of Greater Manchester city regional VSOs was lower across all of Greater Manchester (five per cent) than it was in Manchester itself (14 per cent), where many of these organisations are likely to be headquartered.

Figure 3.2: Type of organisations

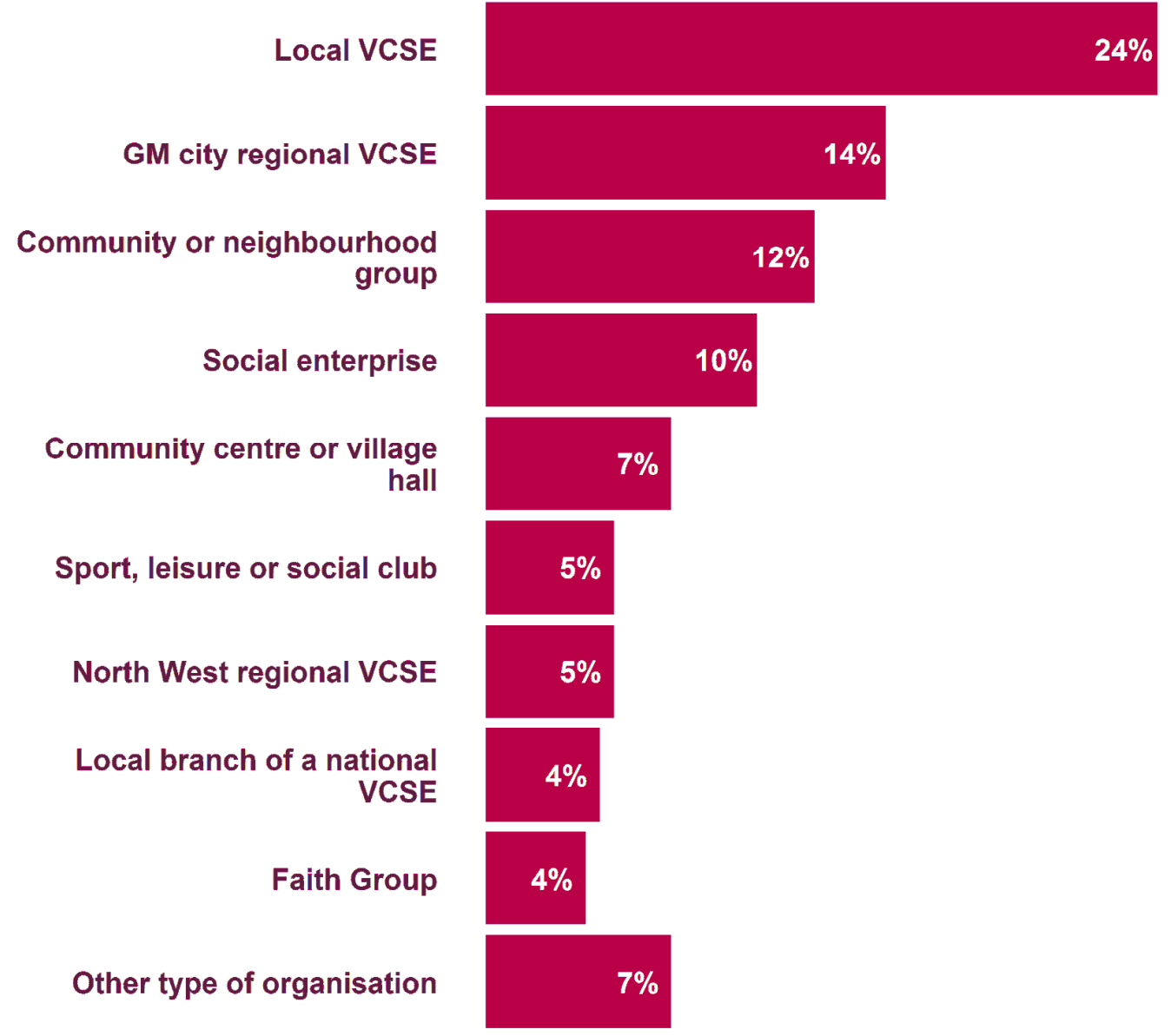

Source: City of Manchester State of the VCSE sector survey 2016/17 Base: 198

\subsection{How long have organisations in the VCSE sector been operating?}

The questionnaire asked respondents to indicate when their organisation was formed. Assessment of organisations by the year in which they were formed provides an indication of how established the VCSE sector is in the City of Manchester.

The responses received build a picture of a VCSE sector that has a fairly well established core. However, the VCSE sector in the City of Manchester has also seen the formation of many new organisations since 2001. Figure 3.3 shows that 60 per cent of organisations responding to the survey had been formed since 2001, including 43 per cent in the past 10 years (i.e. since 2006). Furthermore, an additional 13 per cent were formed between 1991 and 2000; this means around 
three-quarters (73 per cent) of organisations were formed in the last 25 years. At the other end of the spectrum nine per cent of organisations had been formed before 1971, including five per cent formed in 1910 or before.

In the 2012/13 survey, 62 per cent of organisations were formed since 1991, including 38 per cent, which had been formed in the past 10 years. Seven per cent of organisations in 2012/13 had been formed in 1910 or before, compared with five per cent in the 2016/17 survey.

The pattern for organisations responding to all of the Greater Manchester surveys was broadly similar. 43 per cent of respondents had been formed in the past 10 years, although a slightly lower figure, 29 per cent of organisations had been formed since 2011. Six per cent of Greater Manchester organisations had been formed before 1911.

Figure 3.3: Year in which organisations were formed

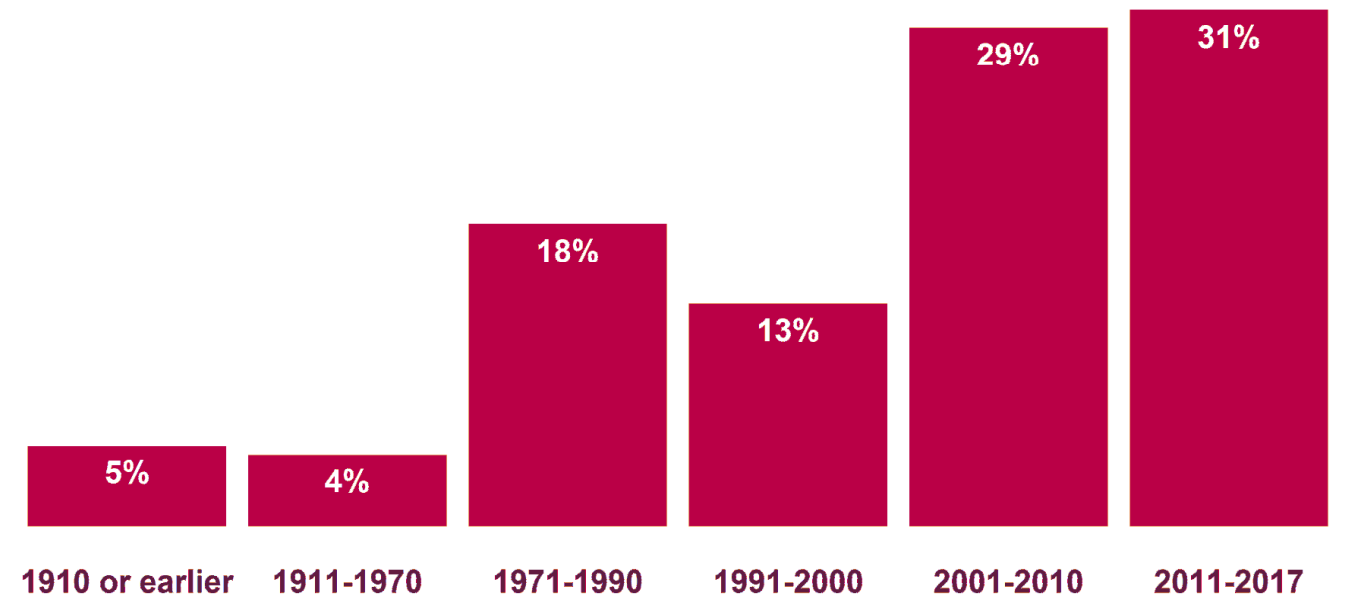

Source: City of Manchester State of the VCSE sector survey 2016/17

Base: 190

It is important to conclude this section by drawing an important qualification. Although the results suggest that it is likely that the VCSE sector in the City of Manchester has experienced growth in the number of organisations established in the last 20 years or so, it may not be as dramatic as the figures suggest. By definition, the survey is of organisations still operating in the City of Manchester in 2016/17, not those which have closed down or ceased operations. Of the organisations which have survived through to $2016 / 17$, the results suggest that a high proportion were established in the last 20 years. But some of the organisations established before, and since, may have subsequently closed down. Because we do not know the rate of closure over time we cannot be certain that the aggregate number of organisations being established or surviving is increasing.

\subsection{What does the VCSE sector in the City of Manchester do?}

To elicit a picture of what the VCSE sector in the City of Manchester does, the survey asked respondents to identify up to three main areas in which their organisation operates. Figure 3.4 presents the top ten main areas selected and confirms the message that the VCSE sector in the City of Manchester works in a diverse range of thematic service areas. However, the proportion of responding organisations working in each area varies. This is most likely dependent on need and funding opportunities. 
Figure 3.4 shows:

- 49 per cent worked in community development, more than in the 2012/13 survey (43 per cent); it was the most frequently recorded category in both surveys

- 44 per cent of organisations worked in the area of health and well-being, up slightly from 40 per cent in 2012/13

- $\quad$ other popular areas included education, training and research (33 per cent) and economic well-being (22 per cent)

- the remaining areas within the top ten ranged between ten per cent (capacity building) and 16 per cent (arts, heritage and culture).

Across Greater Manchester as a whole the same three areas of work were reported as being the most common to work within:

- $\quad$ health and well-being (46 per cent)

- community development (39 per cent)

- $\quad$ education, training and research (26 per cent).

Sport and leisure, however, was ranked higher across Greater Manchester as a whole (25 per cent) than it was in the City of Manchester alone (13 per cent). 
Figure 3.4: Top 10 main areas in which organisations work

Community development

Health and well-being

Education, training and research (including information, advice and guidance)

Economic well-being

Arts, heritage and culture

Environment and sustainability

Sport and leisure

Social care

Capacity building and other support for voluntary or community organisations

Other charitable, social or community purpose

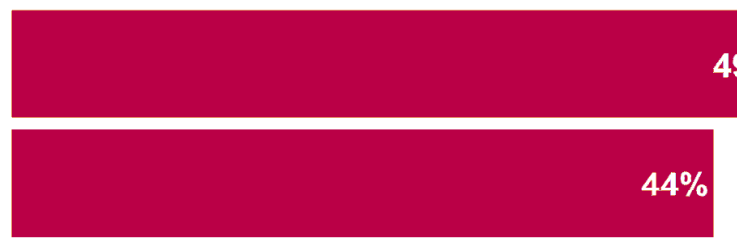

$33 \%$
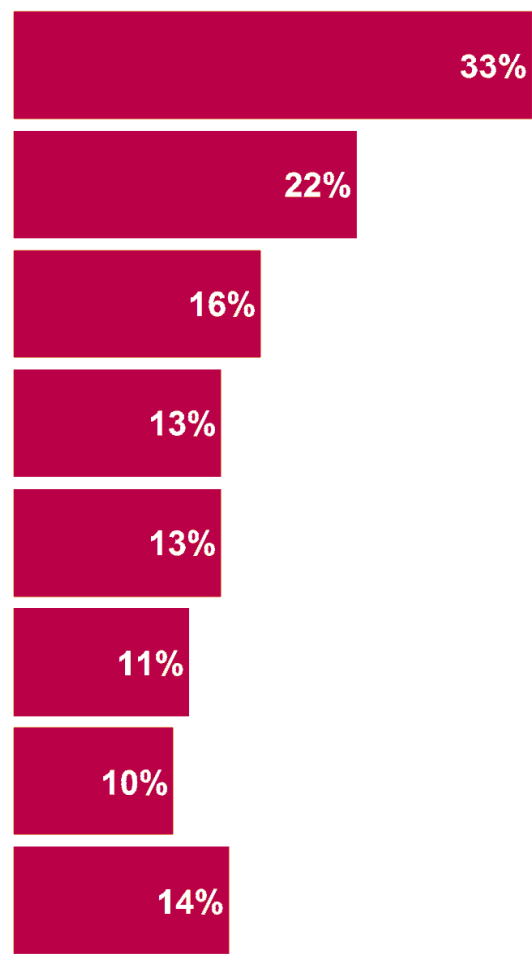

$22 \%$

$16 \%$

$13 \%$

$13 \%$

$11 \%$

$4 \%$

Source: City of Manchester State of the VCSE sector survey 2016/17

Base: 199

In the latest survey respondents who indicated they worked in the area of health and well-being were asked to specify the specific areas in which they operate. The majority (81 per cent) stated they worked in health and well-being in general. Over two-thirds (69 per cent) indicated they worked in mental health. In addition over one third said they work in healthy living (food \& lifestyle, sexual health) (42 per cent). Responses were very similar for Greater Manchester overall, though the percentage that selected mental health was slightly lower (55 per cent versus 69 per cent in the City of Manchester).

In a similar vein, respondents who identified education, training and research as a main area of work were asked to specify the areas they worked within this theme. Almost three-quarters (73 per cent) said they worked in information, advice and guidance and 70 per cent stated they worked in education. Two-thirds (67 per cent) indicated they worked in the area of employability skills. Responses were again similar at the Greater Manchester level.

The ten per cent $(n=20)$ of organisations responding to the survey who indicated that equalities and civil rights was a main area of their work were also asked to identify the specific areas within this category in which they operate. The most common responses were gender ( 63 per cent), age (58 per cent) and disability (47 per cent).

The results are broadly similar to those across Greater Manchester, but a slightly higher proportion of respondents chose the disability category (66 per cent). 


\section{Who the Voluntary}

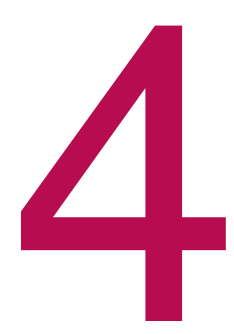

Community and Social Enterprise Sector in the City of Manchester works with

This chapter focuses on who the VCSE sector in the City of Manchester works with and where.

4.1. Who are the clients, users or beneficiaries of the VCSE sector in the City of Manchester?

The questionnaire asked respondents to provide the total number of individual clients, users or beneficiaries that their organisation had supported in the last year, both overall and within the City of Manchester. Analysis of responses to this question by size and type of organisation revealed that in many cases organisations had provided the number of 'interventions' or 'contacts' that they had had with clients, users or beneficiaries. So for example an individual who visited a community centre once a week would have been counted 52 times within the year. Whilst some organisations will have provided the number of unique clients, users or beneficiaries, so as not to overestimate, in our analysis we have assumed the number provided represents the total number of interventions.

Summing across the 181 organisations that responded gives a total of 555,000 interventions overall. Doing the same for the 176 organisations who provided a figure for the City of Manchester gives a total of 427,200 interventions. The responses received can be extrapolated for the estimated 3,394 organisations thought to be operating in the VCSE sector in the City of Manchester to provide an estimate of the total number of interventions by City of Manchester organisations. Working through the calculation it is estimated that City of Manchester organisations had:

\section{1 million interventions with clients, users or beneficiaries in the past year overall}

\section{4 million interventions with clients, users or beneficiaries in the past year in City of Manchester}


The 2012/13 study estimated that City of Manchester organisations made 7.7 million interventions with clients, users or beneficiaries overall. The lower figure found in this latest study could be due to a number of factors. It may reflect genuine changes in the sector, for example interventions becoming more complex, or could also in part be due to revisions to the survey methodology described in Appendix 1. As highlighted above the question asked respondents to provide the total number of individual clients, users or beneficiaries that their organisation had supported in the last year, however, in many cases organisations had provided the number of 'interventions' or 'contacts' that they had had. This inconsistency in response from organisations may also help to explain the different figures reported.

The questionnaire also asked respondents to identify up to three groups that make up the main clients, users or beneficiaries of their organisation.

Figure 4.1 shows that, as might be expected, the VCSE sector in the City of Manchester serves a diverse and wide ranging population. In many cases, client groups are served by relatively small numbers of organisations: 10 per cent of organisations or fewer serve 16 of the client groups listed.

Figure 4.1 shows the client group served by the largest proportion of organisations was everyone (27 per cent). Demographic categories are also served by a larger proportion of organisations, such as children and young adults (26 per cent), black and ethnic minority communities (16 per cent) and women (13 per cent).

Some categories that relate to shared experiences or conditions were also relatively prevalent, such as people with mental health problems (14 per cent), unemployed people (13 per cent) and refugees and people seeking asylum (nine per cent).

The results in 2012/13 were similar, with the most common category indicating organisations worked with everyone (33 per cent), followed by young people (25 per cent) and women (23 per cent).

Analysis of responses to the Greater Manchester survey found a broadly similar pattern with general and demographic client groups also being the most common beneficiary groups identified:

- $\quad$ everyone: 33 per cent

- children and young adults: 23 per cent

- $\quad$ older people: 17 per cent

- $\quad$ women: 15 per cent

- men: 12 per cent. 
Figure 4.1: Top 10 main client groups of City of Manchester organisations

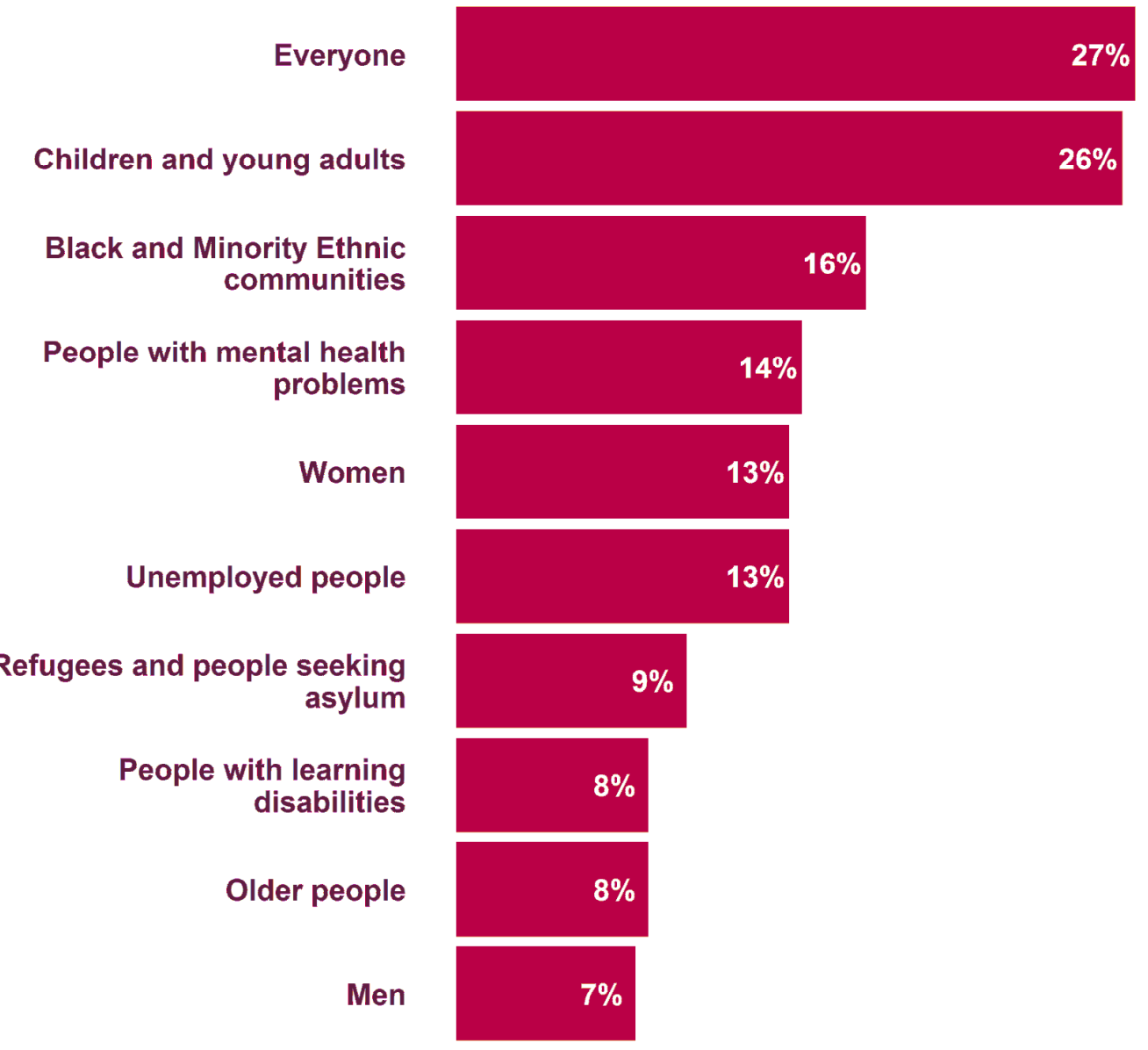

Source: City of Manchester State of the VCSE sector survey 2016/17

Base: 200

Respondents to the $2016 / 17$ survey were asked to identify the ways in which their organisation makes a difference for its service users/client group(s). This question demonstrates the key role that the VCSE sector has in fostering strong and cohesive communities within the City of Manchester and highlights the importance of the VCSE sector as an essential part of the social fabric of the City. As figure 4.2 shows, about three-quarters (74 per cent) of organisations felt they are addressing the needs of disadvantaged members of the community. Other common responses were improving people's mental wellbeing (66 per cent), increasing people's skills (66 per cent) and encouraging people to be involved in regular volunteering (65 per cent).

These categories were also common across Greater Manchester, with over 60 per cent reporting they were: improving people's mental wellbeing (68 per cent), increasing people's skills (60 per cent), and addressing the needs of disadvantaged members of the community (63 per cent). Slightly fewer reported encouraging people to be involved in regular volunteering ( 58 per cent) compared to the City of Manchester alone. 
Figure 4.2: Top 10 ways in which organisations make a difference

Addressing the needs of disadvantaged members of the community

Improving people's mental wellbeing

Increasing people's skills

Encouraging people to be involved in regular volunteering

Encouraging people to get involved in activities and events that are happening

Helping people to feel that they belong to their neighbourhood

Helping people from different backgrounds to get on well together

Improving people's physical wellbeing

Helping people to feel safe

Helping people feel that Manchester is a good place to live

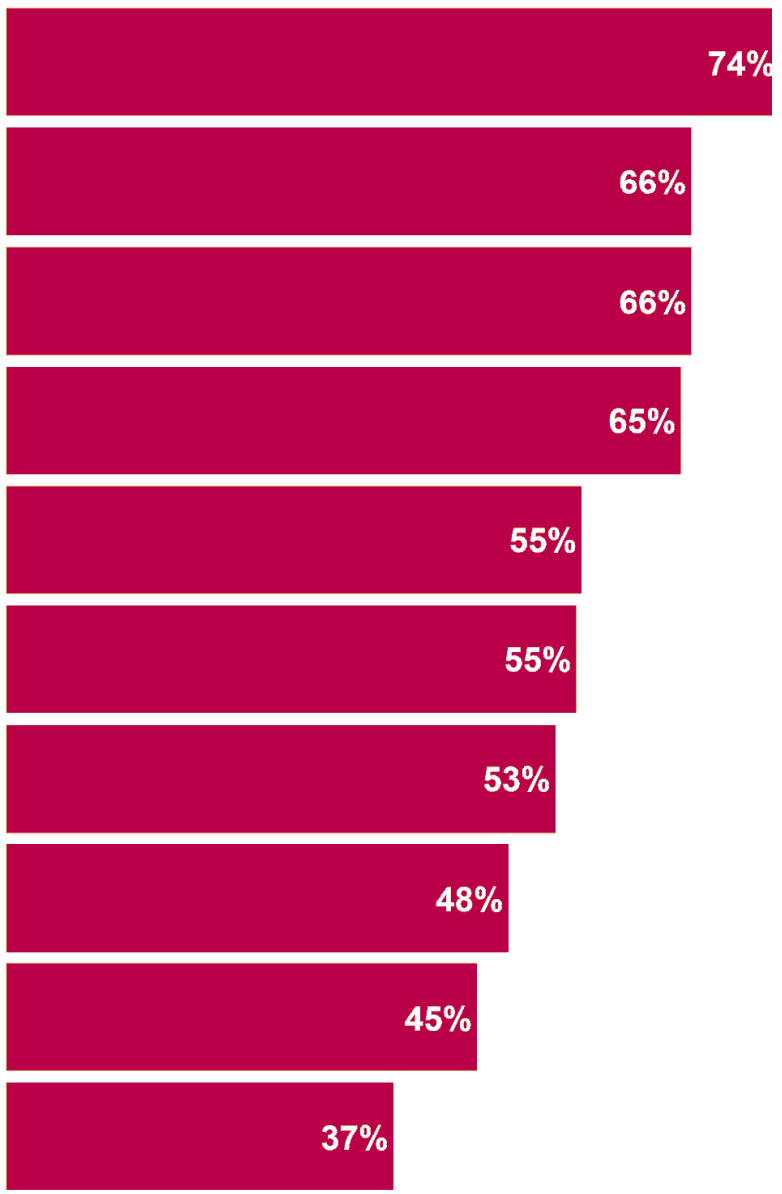

Source: City of Manchester State of the VCSE sector survey 2016/17 Base: 199

\subsection{What geographical levels does the VCSE sector operate at?}

The survey asked respondents to identify the main geographical levels at which they operate - this ranged from the neighbourhood level, to those operating across England, the UK or overseas ${ }^{14}$. In this question respondents were asked to pick out up to three main geographic levels, the results of which are presented in figure 4.3. This shows that the local area is a main focus for a majority of organisations:

- 42 per cent of responding organisations identified particular Manchester neighbourhoods and communities as a main focus of their work; down compared to the $2012 / 13$ survey (53 per cent), though it was the highest ranked category in both cases

- a further 23 per cent identified themselves as having a main focus of the Manchester Local Authority Area as a whole; again somewhat lower than in the 2012/13 survey (34 per cent).

\footnotetext{
${ }^{14}$ This question was asked slightly differently in the latest survey compared to 2012/13. Two additional options ('Across more than one Greater Manchester Local Authority area' and 'Across the whole of Greater Manchester') were included.
} 
A modest proportion of organisations cited that a main geographic area at which they worked was either national (16 per cent) or international (five per cent). In many cases those organisations that work internationally will reflect their main clients, users and beneficiaries.

The picture for Greater Manchester organisations shows that 44 per cent identified particular neighbourhoods and communities as a main geographic focus, a similar proportion as in the City of Manchester. The percentage of organisations who said they worked nationally and internationally was similar within the City of Manchester and Greater Manchester.

Figure 4.3: Main geographic focus

\section{Particular Manchester neighbourhoods and communities}

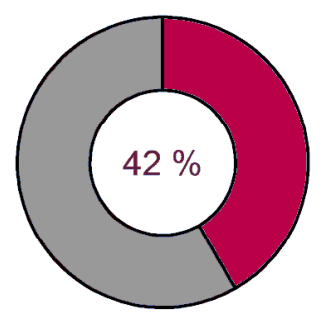

Regionally across the North West

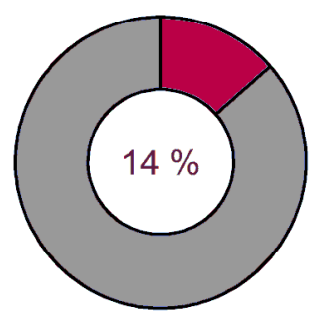

Across the whole Manchester Local Authority area

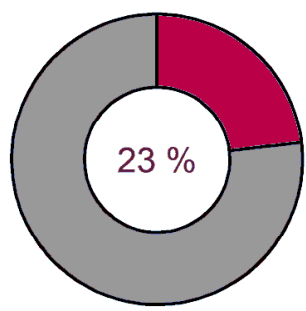

Nationally

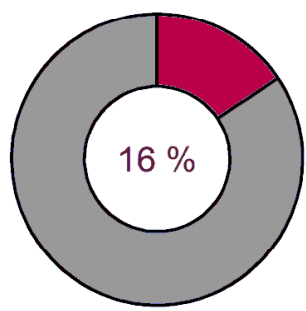

Across more than one

Greater Manchester

Local Authority area

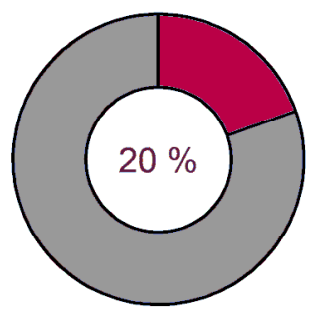

Internationally

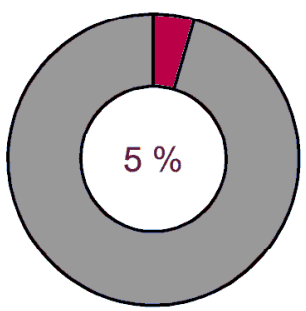

Across the whole of Greater Manchester

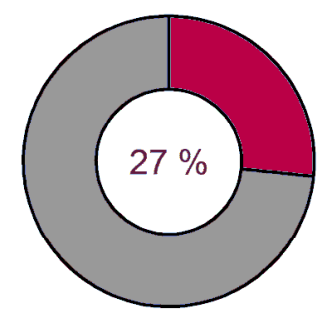

Source: City of Manchester State of the VCSE sector survey 2016/17

Base: 199

Using the responses to this question it is also possible to identify the highest main geographic area that an organisation carries out its activities (see figure 4.4 below). The highest geographic area that could be identified was internationally.

This analysis finds that for just under one third (32 per cent) their highest main geographic focus was particular City of Manchester neighbourhoods or communities; similar to the proportion in 2012/13 (40 per cent). This is almost identical to Greater Manchester as a whole where 34 per cent of organisations indicated their highest main geographic focus was particular neighbourhoods and communities. 
Figure 4.4: Highest geographic focus
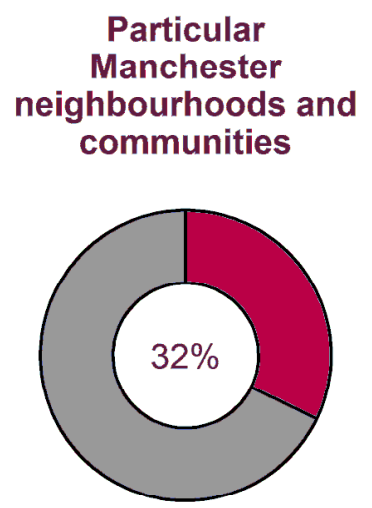

Regionally across the North West

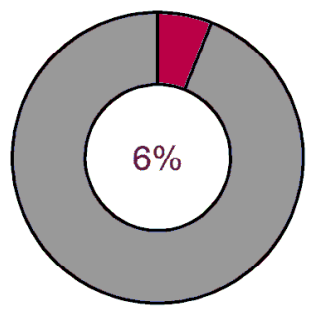

Across the whole Manchester Local Authority area

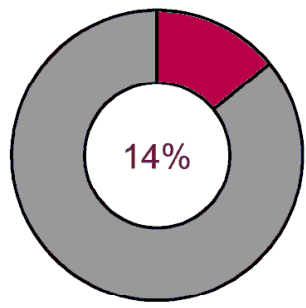

Nationally

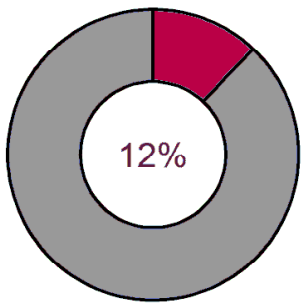

Across more than one Greater Manchester Local Authority area

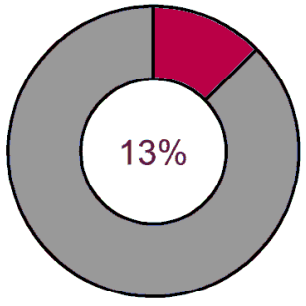

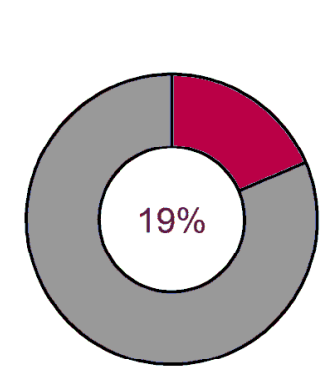

Across the whole of Greater Manchester
Internationally

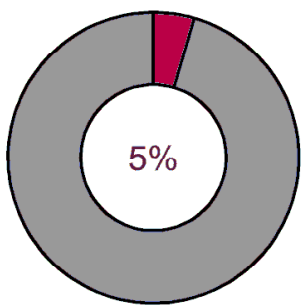

Source: City of Manchester State of the VCSE sector survey 2016/17

Base: 199

Respondents who reported that the whole City of Manchester local authority area or particular City of Manchester neighbourhoods or communities were a main geographic focus of their organisation were asked to identify in which wards their work focused on. Map 4.1 shows the percentage of all organisations that identified each of the City of Manchester's wards as a main focus of their work.

30 per cent of City of Manchester organisations identified Levenshulme as a main focus of their work, the same number as identified Longsight. In addition between 20 and 30 per cent of City of Manchester organisations identified Hulme, Moss Side, Rusholme, Fallowfield and Northenden as a main focus.

The four wards which were a main focus for the lowest proportions of City of Manchester organisations were Old Moat, Charlestown, Bradford and Brooklands, all a main focus for less than 10 per cent of City of Manchester organisations. 
Map 4.1: Percentage of organisations that identify City of Manchester wards as a main focus of their work

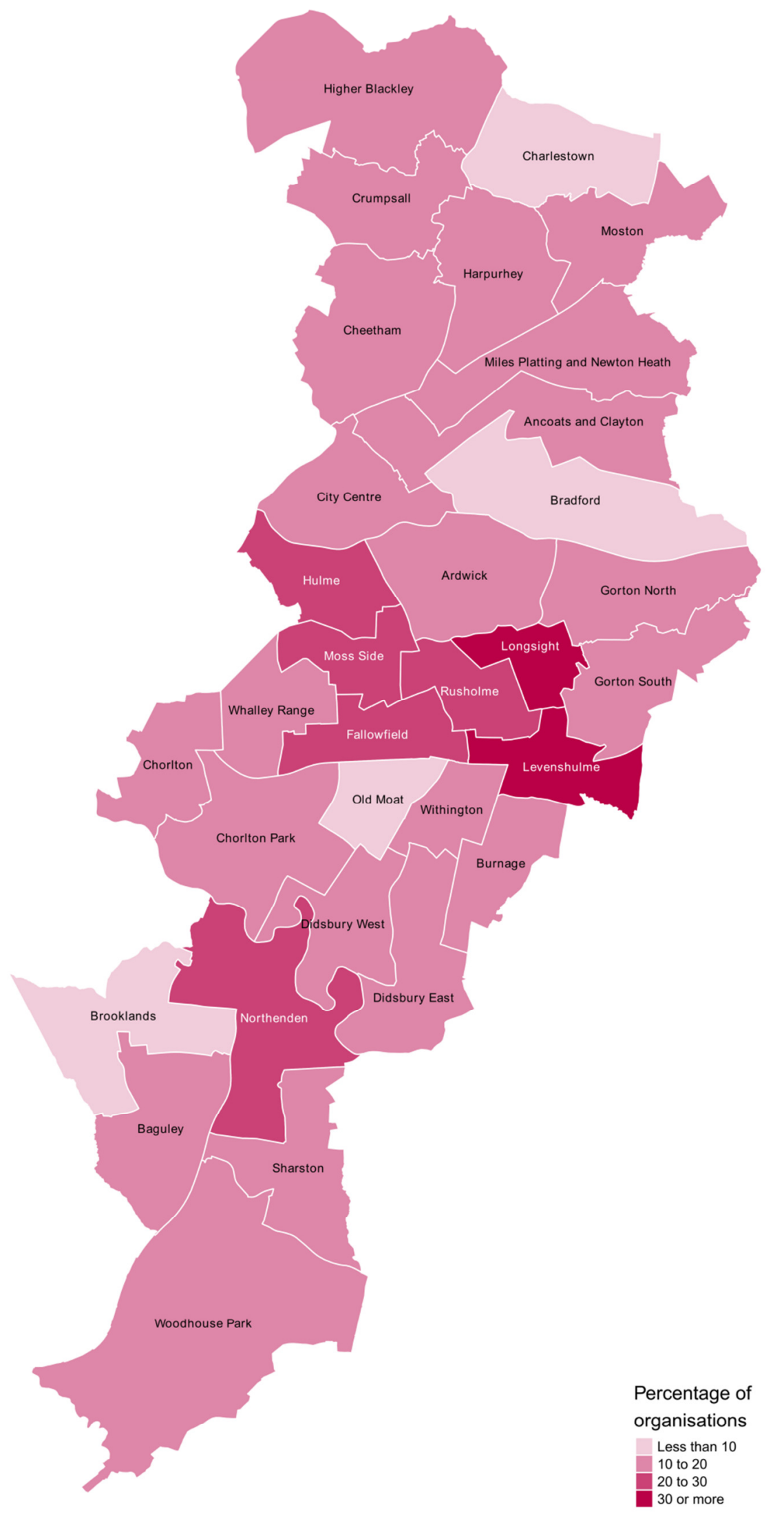

Source: City of Manchester State of the VCSE sector survey 2016/17 Base: 83 


\section{Finances and Income}

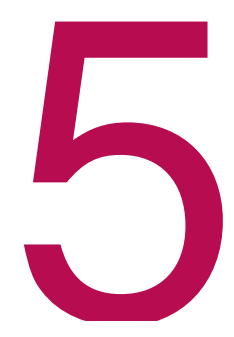

This chapter provides an overview of the finances and income of the VCSE sector in the City of Manchester. It includes estimates of the overall income received by the sector between 2012/13 and 2014/15, analysis of the different sources of income received (public sector and non-public sector) and their relative contribution, and an assessment of the financial sustainability of the VCSE sector.

Where possible this chapter compares results from the latest survey and the 2012/13 study. Revisions to the questionnaire and methodology between these studies, however, mean that comparisons are not always possible or appropriate and that caution should be applied when comparing across the two waves (see Appendix 1 for more detail).

\subsection{Income}

Based on the average (mean) income of respondents to the survey across Greater Manchester, and drawing on the assumptions used to estimate the total number of organisations in the City of Manchester, the following is estimated ${ }^{15}$ -

\section{$£ 526$ million the total income of the VCSE sector in the City of Manchester in 2014/15}

This total income estimate is higher than the figure of $£ 477$ million estimated for the sector in 2011/12 from the 2012/13 survey. As discussed in Chapter 3 exploring the distribution by size category based on income for 2014/15 across Greater Manchester showed some inconsistency with the distribution found in 2013. This could explain some of the difference in the estimates above. The latest income figure provides a more robust estimate than the earlier study as it draws upon data from both waves of the survey, and therefore a more comprehensive contact list of organisations, to calculate the proportion of organisations estimated to be in each size category.

The estimate for 2014/15 also represents an increase of three per cent compared to $2013 / 14$ when the total income of the VCSE sector was an estimated $£ 509$ million. This follows a reduction between 2012/13 and 2013/14 of an estimated six per cent in the total income of the sector. This data is outlined in more detail in figure 5.1.

\footnotetext{
${ }^{15}$ This figure is based on a weighted average (mean) for each size category for respondents from across Greater Manchester. The methodology is explained in more detail in the methodological appendix.
} 
Figure 5.1: Estimated annual income of the VCSE sector in the City of Manchester (2012/13-2014/15)

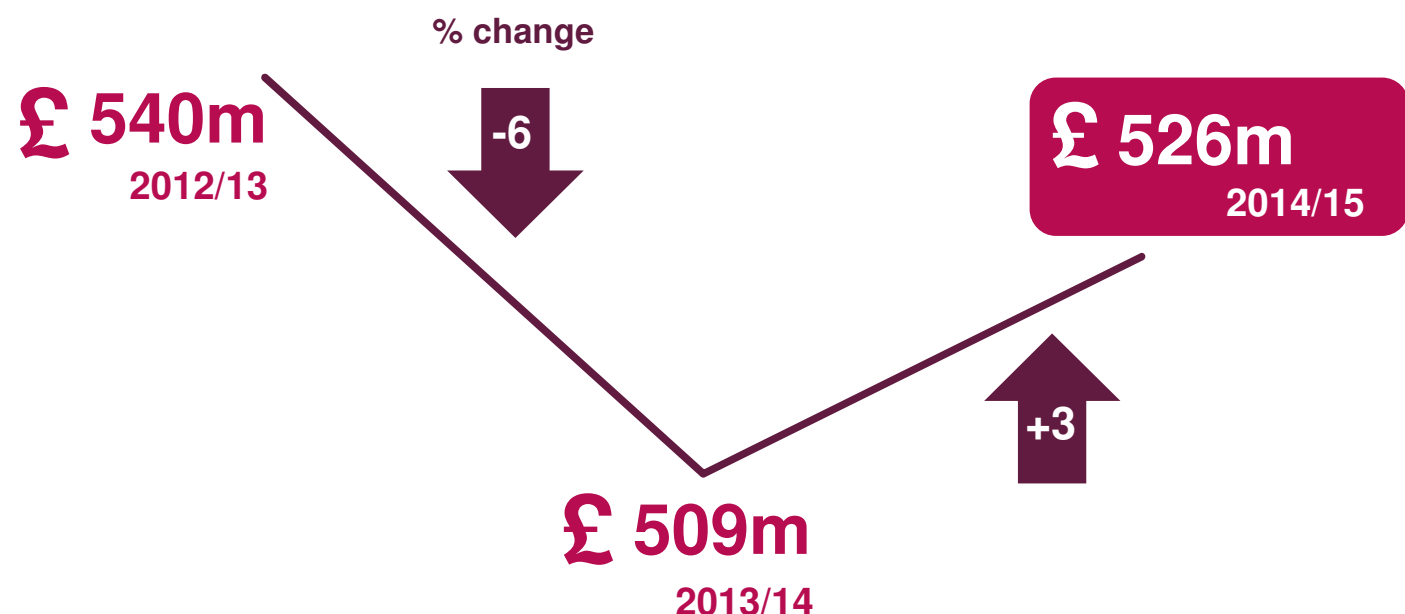

Source: City of Manchester State of the VCSE sector survey 2016/17

Base: 125 All figures are in 2014/15 prices

This change in income should be viewed in the wider national context discussed in Chapter 2. The picture is somewhat more positive than in the previous 2012/13 study. Between 2012/13 and 2013/14 the income and spending of the VCSE sector in the UK increased, representing the first notable net growth since the peaks of 2007/08 and 2009/10 respectively. While the data above shows a decrease in the City of Manchester between these two years results indicate a more recent upturn in the local area. However, with austerity measures set to continue for the foreseeable future and public sector funding for the sector continuing to be squeezed, there is still need for caution.

When the VCSE sector's income is explored in more detail it shows notable variations according to organisation $\operatorname{size}^{16}$. In 2014/15, the majority of income was concentrated in large and medium sized organisations even though the majority of organisations were micro or small. This is outlined in more detail in figure 5.2.

\footnotetext{
${ }^{16}$ In exploring organisation size we used the categories developed by NCVO for use in their Almanac series (see e.g. Clark et al., 2010)
} 
Figure 5.2: Proportion of organisations and proportion of income by organisation size $(2014 / 15)$

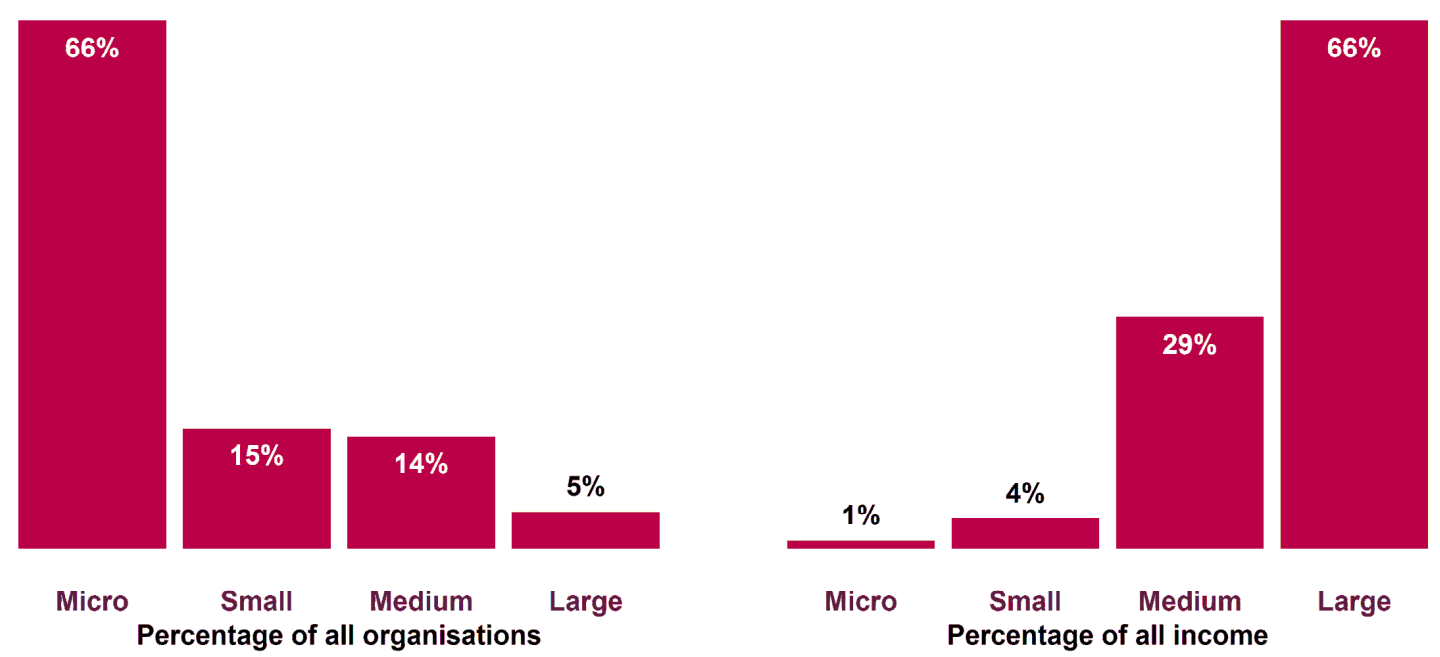

Source: City of Manchester State of the VCSE sector survey 2016/17

Base: 125

This shows that micro and small organisations account for over eight out of ten organisations in the VCSE sector but less than five per cent of total income. By contrast medium and large organisations account for just 19 per cent of the VCSE sector's organisations but receive more than 95 per cent of its income. Income is concentrated particularly in the largest organisations, with two thirds of all income (66 per cent) into the VCSE sector received by only 158 organisations.

Analysis of income data from survey respondents across Greater Manchester ${ }^{17}$ identified further variations according to organisation size when we explored how income levels had changed between 2012/13 and 2014/15. These are summarised in table 5.1 .

Table 5.1: Estimated change in annual income by organisation size (all Greater Manchester organisations: 2012/13-2014/15)

\begin{tabular}{|c|c|c|c|c|c|c|c|c|}
\hline & \multicolumn{2}{|c|}{$\begin{array}{c}\text { Micro } \\
\text { (under } £ 10 k \text { ) }\end{array}$} & \multicolumn{2}{|c|}{$\begin{array}{c}\text { Small } \\
(£ 10 k-£ 100 k)\end{array}$} & \multicolumn{2}{|c|}{$\begin{array}{c}\text { Medium } \\
(£ 100 k-£ 1 m) \\
\end{array}$} & \multicolumn{2}{|c|}{$\begin{array}{c}\text { Large } \\
\text { (more than } £ 1 \mathrm{~m} \text { ) }\end{array}$} \\
\hline & Income & $\begin{array}{c}\% \\
\text { change }\end{array}$ & Income & $\begin{array}{c}\% \\
\text { change }\end{array}$ & Income & $\begin{array}{c}\% \\
\text { change }\end{array}$ & Income & $\begin{array}{c}\% \\
\text { change }\end{array}$ \\
\hline $2012 / 13$ & $£ 32.3 \mathrm{~m}$ & & $£ 84.9 \mathrm{~m}$ & & $£ 413.9 \mathrm{~m}$ & & $£ 829.2 \mathrm{~m}$ & \\
\hline $2013 / 14$ & $£ 31.0 \mathrm{~m}$ & -4 & $£ 82.4 \mathrm{~m}$ & -3 & $£ 382.8 \mathrm{~m}$ & -8 & $£ 785.1 \mathrm{~m}$ & -5 \\
\hline $2014 / 15$ & $£ 30.0 \mathrm{~m}$ & -3 & $£ 77.0 \mathrm{~m}$ & -7 & $£ 391.5 \mathrm{~m}$ & 2 & $£ 822.6 \mathrm{~m}$ & 5 \\
\hline
\end{tabular}

Source: Greater Manchester State of the VCSE sector survey 2016/17

Base: 720 All figures are in 2014/15 prices

This shows that across Greater Manchester the micro and small organisation categories experienced year on year reductions in total income between 2012/13 and $2014 / 15$. For micro organisations this is a continuation of a trend identified in the 2012/13 survey where these organisations experienced a reduction of 12 per cent between 2010/11 and 2011/12. In contrast the 2012/13 survey identified a small increase in income between 2010/11 and 2011/12 for small organisations (three per cent).

\footnotetext{
${ }^{17}$ It was not possible to undertake sufficiently robust analysis of these trends at a local authority level
} 
By contrast medium and large organisations saw a reduction in total income between 2012/13 and 2013/14 but then an increase between 2013/14 and 2014/15. For medium organisations this could indicate the start of a reversal in a trend identified in the 2012/13 survey where year-on-year reductions in income were identified (a reduction of four per cent between 2010/11 and 2011/12). This income volatility is a significant challenge in the operating context for medium and large organisations.

\subsection{Sources of Income}

\subsubsection{Public sector income}

Survey respondents were asked to identify the public sector bodies from which they received funding in their most recent financial year. Overall, 71 per cent of respondents reported having at least one source of public sector funds. This is higher than both the 66 per cent who reported having public sector funds in the 2012/13 survey and the figure for Greater Manchester as a whole (68 per cent).

Respondents were asked to identify which specific public organisations they received at least some funding from. The results suggest that by far the most common source of public sector funding is Manchester City Council, reported as a funder by over half (52 per cent) of all City of Manchester respondents. This pattern was reflected across Greater Manchester, where local authorities consistently emerged as the most frequent source of public sector funds. The next most common public sector funding source was NHS Manchester Clinical Commissioning Group (11 per cent). All the remaining sources were identified as funders by less than 10 per cent of respondents.

Respondents were also asked to estimate the proportion of their group or organisation's total income that each source of public sector income represented. Figure 5.3 shows the two most common sources of public sector funding received and the estimated proportion of total income this represents.

Figure 5.3 shows that of the organisations which reported Manchester City Council as a funder:

- 23 per cent said funding from the Council accounted for less than 10 per cent of their total income

- 27 per cent said funding from the Council accounted for at least 10 per cent but less than 20 per cent of their income

- 34 per cent said funding from the Council accounted for at least 20 per cent but less than 50 per cent of their income.

The survey also asked respondents with public sector income whether they had a formal funding agreement for each source. Of the two most commonly identified sources:

- 96 per cent of funds received by organisations from Manchester City Council were supported by a formal agreement

- 82 per cent of funds received by organisations from NHS Manchester CCG were supported by a formal agreement.

Respondents who indicated they had at least one formal agreement in place were asked if any of these were based on payment by results. Almost one third (30 per 
cent) indicated that at least one of their agreements was based on a payment by results basis.

Figure 5.3: Public sector funds received by City of Manchester respondents $(2014 / 15)$

Manchester City Council

NHS Manchester Clinical Commissioning Group

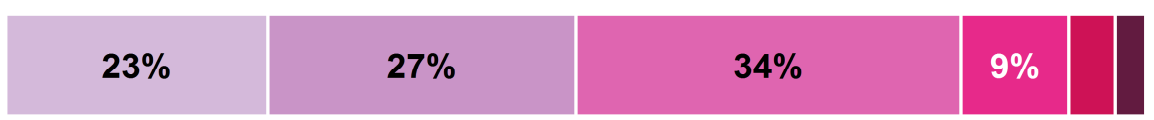

$33 \% 33 \%$

Less than $10 \%$

At least $10 \%$ but less than $20 \%$
At least $20 \%$ but less than $50 \%$
At least $50 \%$ but less than $75 \%$
At least $75 \%$ but less than $100 \%$

Source: City of Manchester State of the VCSE sector survey 2016/17

Base: 74 (Manchester City Council); 15 (NHS Manchester CCG)

The survey also revealed variations in public sector income received by organisations of different sizes. In 2012/13, micro organisations were less likely than small, medium and large organisations to have at least one source of public sector income. In 2016/17 the picture has shifted somewhat towards a more even distribution, as shown in figure 5.4 .

This shows that 71 per cent of micro organisations that responded to the survey received public sector funding compared to 64 per cent of small organisations, 87 per cent of medium organisations and 78 per cent of large organisations. The percentage of micro organisations receiving public sector funding is 27 percentage points higher than in 2012/13, while the percentage of small organisations is 10 percentage points lower.

Figure 5.4: Proportion of City of Manchester organisations in receipt of public sector funds by organisation size (2014/15)

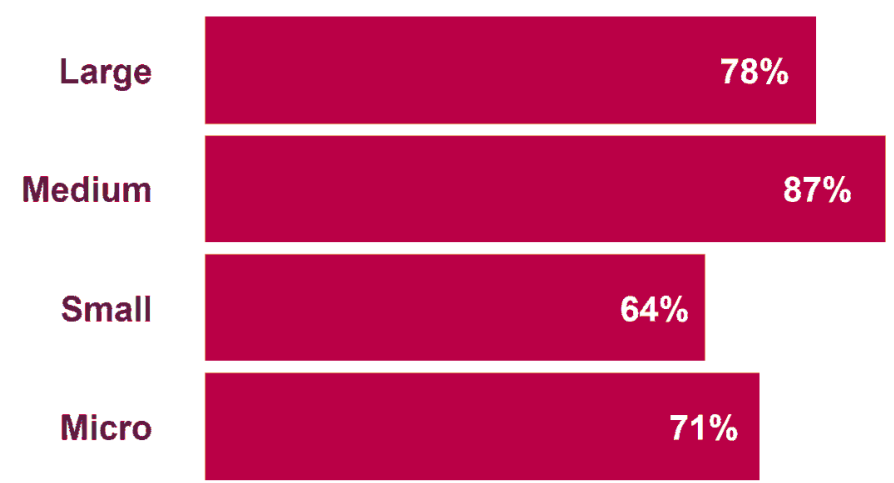

Source: City of Manchester State of the VCSE sector survey 2016/17 Base: 121

\subsubsection{Other sources of income}

Survey respondents were also asked to identify any other sources of income (i.e. non-public sector) they received in 2014/15. Overall, 89 per cent of respondents received funds from at least one non-public sector source. This is a noticeable 
increase of 18 percentage points compared to 2012/13. Across Greater Manchester 84 per cent of respondents received non-public sector income.

Grants from charitable trusts and foundations were identified most frequently as a source of other funds (58 per cent of respondents) followed by fundraising ( 52 per cent) and grants from national lottery distributers ( 45 per cent) and then charging for goods and services (42 per cent). The proportion of respondents identifying these sources across Greater Manchester was generally lower than in the City of Manchester, though the percentage identifying fundraising as a funding source was similar (50 per cent).

Respondents were also asked to estimate the proportion of their group or organisation's total income received from each of the non-public sector funding sources. Figure 5.5 shows the most prominent sources of non-public sector funding received and the estimated proportion of total income this represents.

Figure 5.5 shows that of those receiving the most common source of non-public sector funding, grants from charitable trusts and foundations (81 respondents), just 19 per cent relied on it for more than 50 per cent or more of their income. The equivalent figure for the second most common source, fundraising (70 respondents), is only three per cent.

Figure 5.5: Other funds received by City of Manchester respondents (2014/15)

Grants from charitable trusts and foundations

Fundraising (e.g. crowdfunding events, donations etc.)

Grants from National Lottery distributors (e.g. BIG)

Charging for goods and services

Business donations or sponsorship

Membership fees I subscriptions

Interest (e.g. bank, endowments, investments)
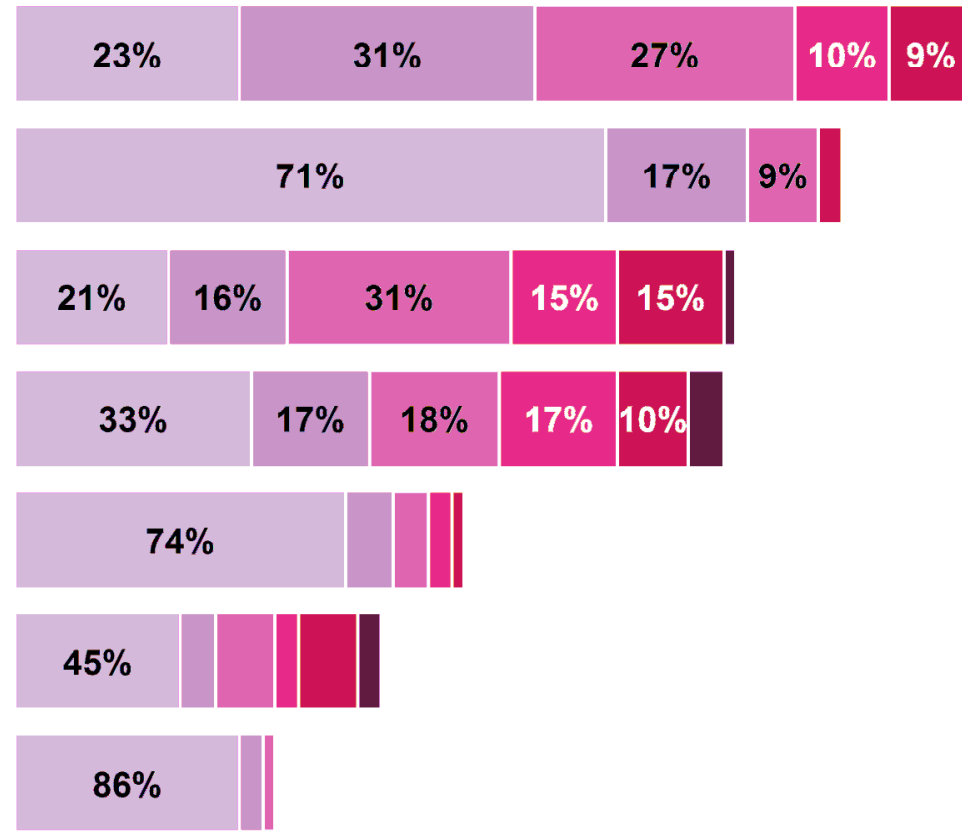

Less than $10 \%$

At least $10 \%$ but less than $20 \%$

Source: City of Manchester State of the VCSE sector survey 2016/17 Base: 22-81

As shown in figure 5.6, all the large and medium organisations surveyed received some form of funding from non-public sector sources. Small and micro organisations were slightly less likely to receive at least one non-public sector funding source (94 and 86 per cent respectively). In all the size categories the percentages are higher than in 2012/13, when only 48 per cent of micro organisations received some form of 
non-public funding. This may suggest that organisations across the board have since diversified their funding models.

Figure 5.6: Proportion of organisations in receipt of other funds by organisation size $(2014 / 15)$

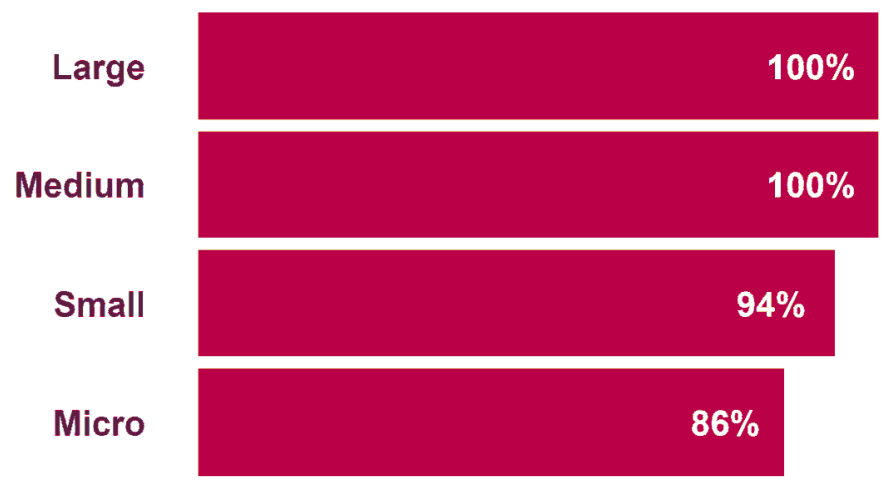

Source: City of Manchester State of the VCSE sector survey 2016/17 Base: 120

\subsection{Financial Sustainability}

The survey asked respondents about how their organisation's financial situation had changed in the past 12 months (i.e. during the current financial year). The results are outlined in figure 5.7 .

Figure 5.7: Change in financial circumstances in the last 12 months

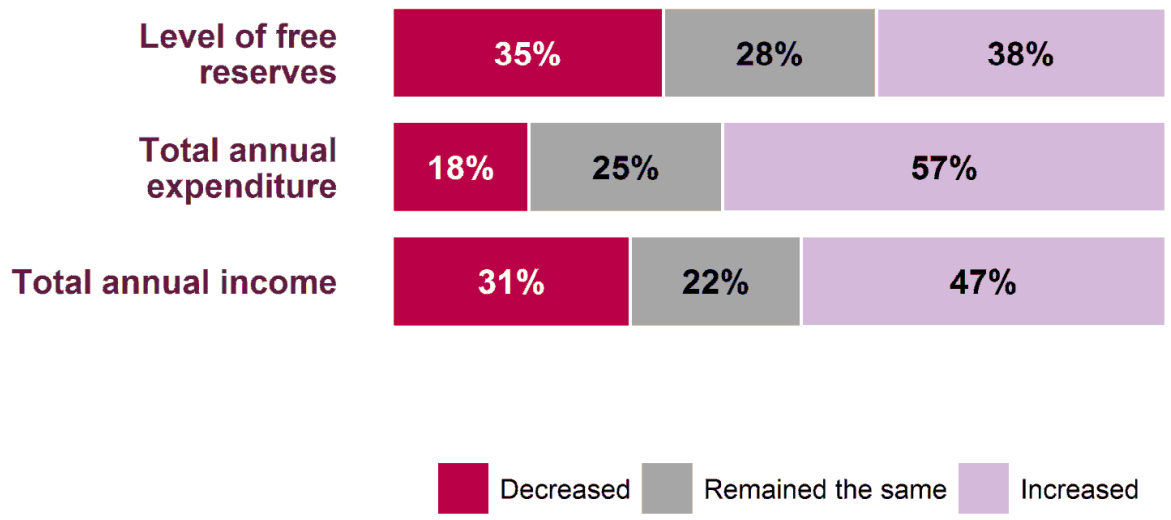

Source: City of Manchester State of the VCSE sector survey 2016/17

Base: 140 (income), 136 (expenditure), 120 (free reserves)

Note: 'cannot say' response has been excluded from the analysis

This raises some concerns: 57 per cent of respondents reported increasing their expenditure but only 47 per cent had experienced an increase in income and only 38 per cent reported an increase in reserves. In addition, 31 per cent of respondents reported a decrease in income but only 18 per cent reduced their expenditure.

20 per cent of respondents provided an expenditure figure for $2014 / 15$ that was greater than their income. This means that there were a notable number of organisations that spent more money than they received in the past $\mathbf{1 2}$ months. This figure is, however, lower than the 30 per cent of organisations who provided an expenditure figure in the 2013 study that was greater than their income, nevertheless 
it still appears that the sustainability of a sizeable number of organisations could be under threat.

Figures $5.8 \mathrm{a}$ and $5.8 \mathrm{~b}$ show that within each of the size categories a higher proportion of organisations increased their expenditure than increased their income. Large organisations were particularly likely to have increased their expenditure, with 75 per cent reporting an increase, while just 50 per cent reported an increase in income.

Figure 5.8a: Change in income in the last 12 months by organisation size

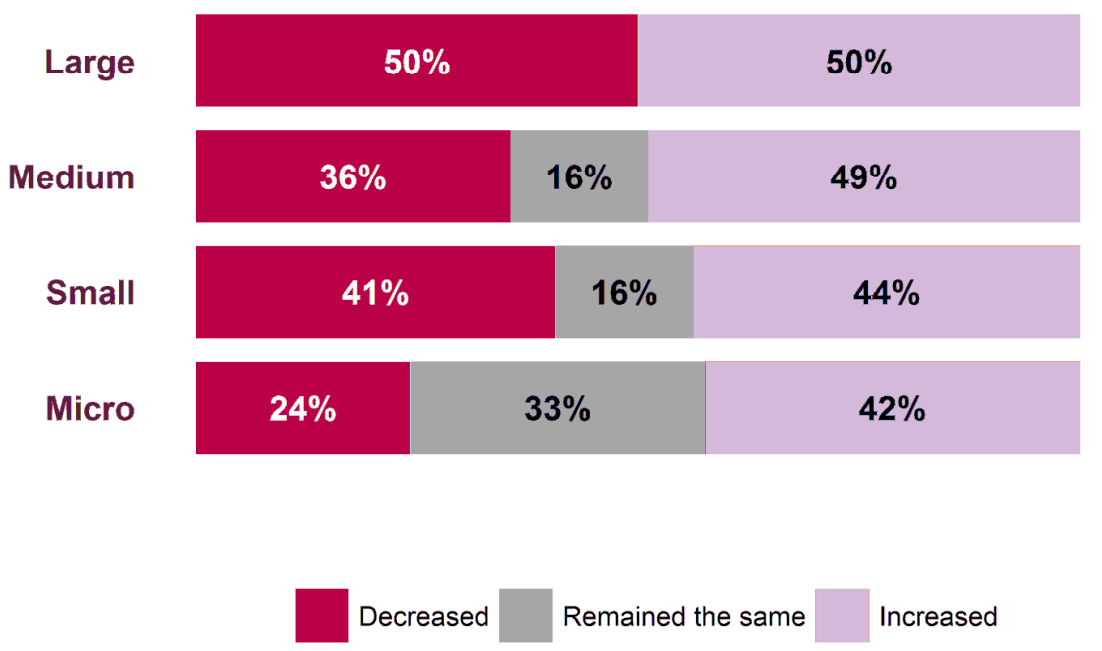

Source: City of Manchester State of the VCSE sector survey 2016/17

Base: 118

Note: 'cannot say' response has been excluded from the analysis

Figure 5.8b: Change in expenditure in the last 12 months by organisation size

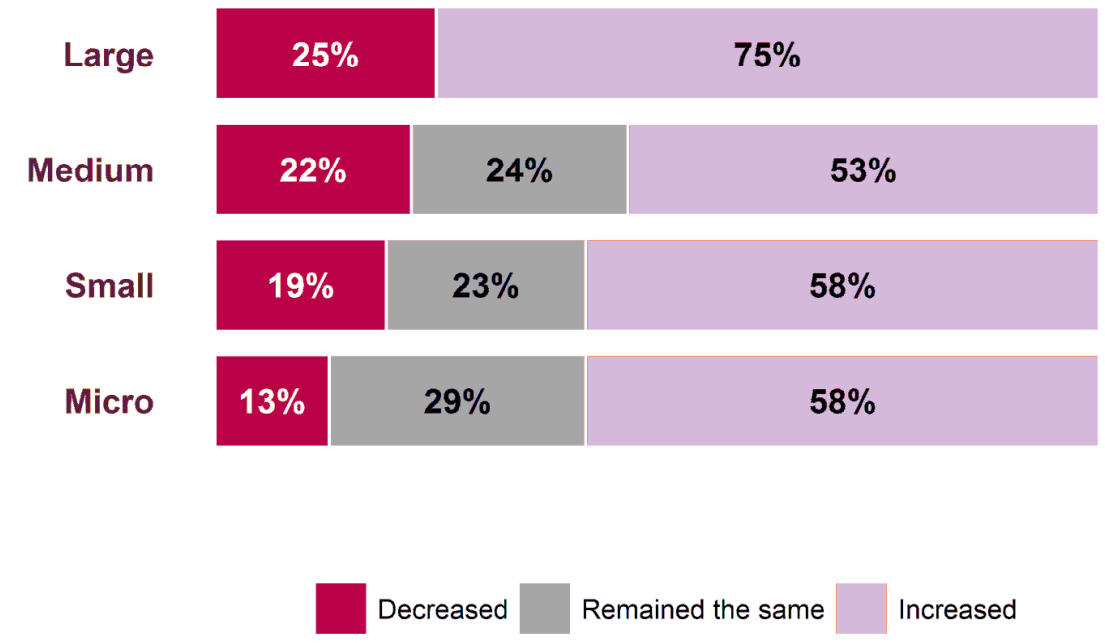

Source: City of Manchester State of the VCSE sector survey 2016/17

Base: 115

Note: 'cannot say' response has been excluded from the analysis

Further analysis of the financial reserve levels reported by respondent organisations provides an additional insight in to the financial health of the VCSE sector. Reserves are important as they provide organisations with funds to fall back on in the short term should other sources of funding reduce or be withdrawn. They also provide 
organisations with the flexibility to develop new and innovative activity that might not have attracted external funding from the outset. Organisations with low reserves relative to expenditure are therefore more likely to be restricted in their ability to adapt if key external funding is lost. In order to explore this issue in more detail reserves (2014/15) were calculated as a proportion of expenditure $(2014 / 15)$ for each respondent. The results are shown in figure 5.9.

\section{Figure 5.9: Financial vulnerability of organisations in the City of Manchester}

Less than 1 month expenditure in reserve

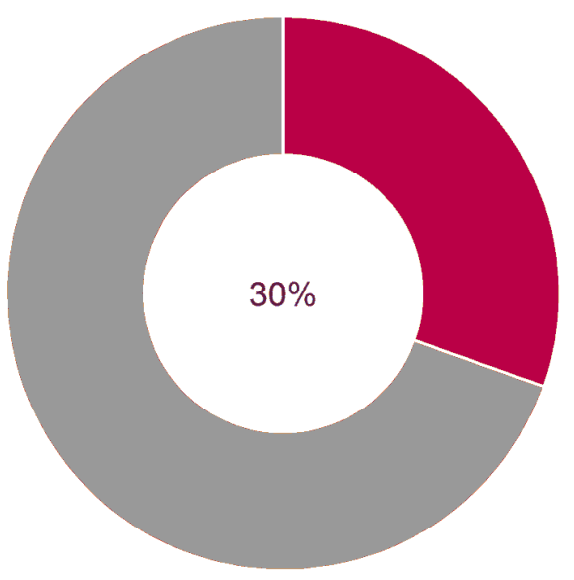

Less than 3 months of expenditure in reserve

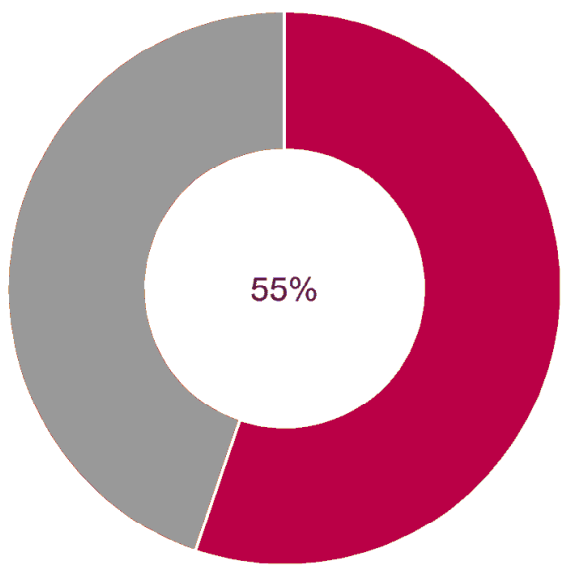

Source: City of Manchester State of the VCSE sector survey 2016/17

Base: 105

This shows that $\mathbf{3 0}$ per cent had reserve levels of less than one month's expenditure, and a further 25 per cent had reserves that covered less than three month's expenditure. This suggests that over half of all organisations in the VCSE sector could be vulnerable should their funds be severely reduced or withdrawn. Proportions are higher than in 2012/13 survey when 16 per cent reported less than one month's expenditure in reserve and 49 per cent in total reported less than three month's reserves. The pattern was similar to that identified at the Greater Manchester level, although the proportion with less than three months expenditure in reserves in the City of Manchester was higher than the 46 per cent across Greater Manchester as a whole and the highest proportion across all the boroughs participating in the study.

Survey respondents were also asked how they thought the environment for funding/income for the VCSE sector will change over the next year. Figure 5.10 shows the responses received to this question. This shows that a majority (65 per cent) of organisations in the City of Manchester thought the environment will deteriorate compared to just six per cent who felt the environment is set to improve. 15 per cent saw the environment for funding/income staying the same. These results were slightly more pessimistic than across Greater Manchester as a whole where 56 per cent thought the environment will deteriorate and seven per cent saw the environment improving. 
Figure 5.10: Change in the environment for funding/income in the next year

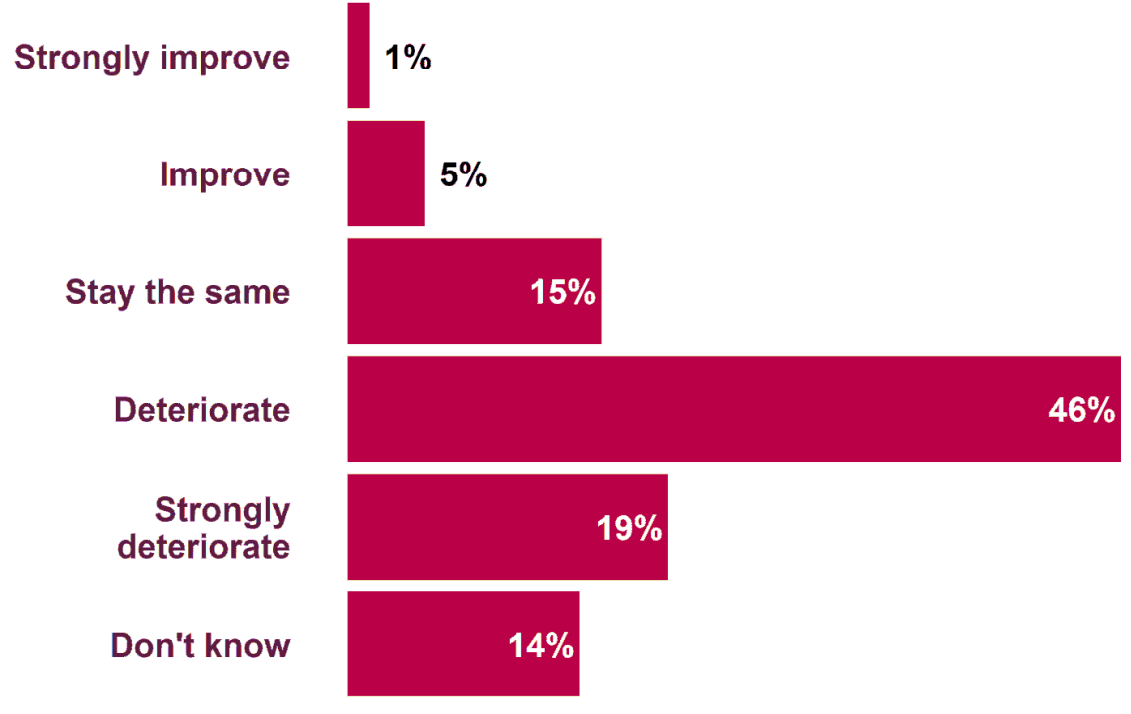

Source: City of Manchester State of the VCSE sector survey 2016/17

Base: 152 


\section{Paid Employees}

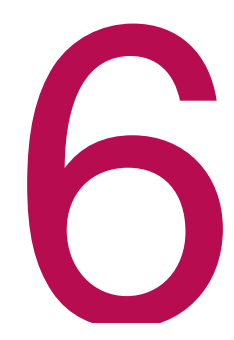

This chapter looks at the paid workforce of the VCSE sector in the City of Manchester.

6.1. How many FTE paid staff are employed in the VCSE sector in the City of Manchester?

Based on the average number of FTE paid staff employed by organisations responding to the survey across Greater Manchester, and drawing on the assumptions used to estimate the total number of organisations in the City of Manchester, it is estimated that:

\section{1,000 FTE paid staff were employed in the VCSE sector in the city of Manchester in 2016/17}

This represents 16,300 employees.

This was 38 per cent of the estimated total number of FTE paid staff working within the VCSE sector in Greater Manchester.

This is a lower figure than the 12,400 FTE paid staff estimated to work in the sector in the 2013 study, which represented 17,200 employees. This lower figure could be due to a number of factors. It may reflect genuine changes in the sector, for example fewer staff working in the sector or more staff working part-time, or could also in part be due to revisions to the survey methodology described in Appendix 1. The inconsistency in the distribution of organisations in the sector by size category found between the two waves of the survey could also be a factor (see Appendix 1 for more detail).

Gross Value Added (GVA), the value of goods and services produced, is a key measure of the economic contribution of organisations or sectors. It can be estimated for paid employees working in City of Manchester organisations by multiplying the number of FTE paid staff by the estimated gross value added (GVA) per FTE employee ${ }^{18}$. From this calculation it is estimated:

\section{$£ 347.0 \mathrm{~m}$ contributed to the economy per annum by paid employees of City of Manchester VCSE sector organisations}

\footnotetext{
${ }^{18}$ This study used Greater Manchester GVA per employee averaged across the following two VCSE sectors: education and human health and social work activities.
} 
Three-fifths (61 per cent) of FTE paid staff were employed in large organisations with an income of at least one million pounds. In comparison the 2,759 micro and small organisations employed just eight per cent of FTE paid staff. The remaining 30 per cent were employed in medium sized organisations. As in 2012/13, large and medium sized organisations especially continue to be significant employers in the City of Manchester.

Figure 6.1 presents a breakdown of responding organisations by the number of FTE paid staff they employed. 69 per cent of organisations employed less than five FTE paid staff members. Included in this figure were 33 per cent of organisations that did not employ any paid staff. Further analysis reveals that the majority of these were micro organisations with income of less than $£ 10,000$. At the other end of the spectrum ten per cent of organisations employed 20 or more FTE paid members of staff. A greater proportion of organisations responding to the 2012/13 survey indicated they did not employ any staff (50 per cent). This is a notable change. It could be due to a genuine decrease in the proportion of organisations in the sector without any paid staff or could also in part be due to a change in the make-up of organisations responding to the survey.

Compared with the Greater Manchester sample as a whole, a higher proportion of organisations within the City of Manchester appeared to have FTE paid staff: 67 per cent in the City of Manchester compared with 49 per cent in Greater Manchester.

Figure 6.1: Organisations by numbers of FTE paid staff

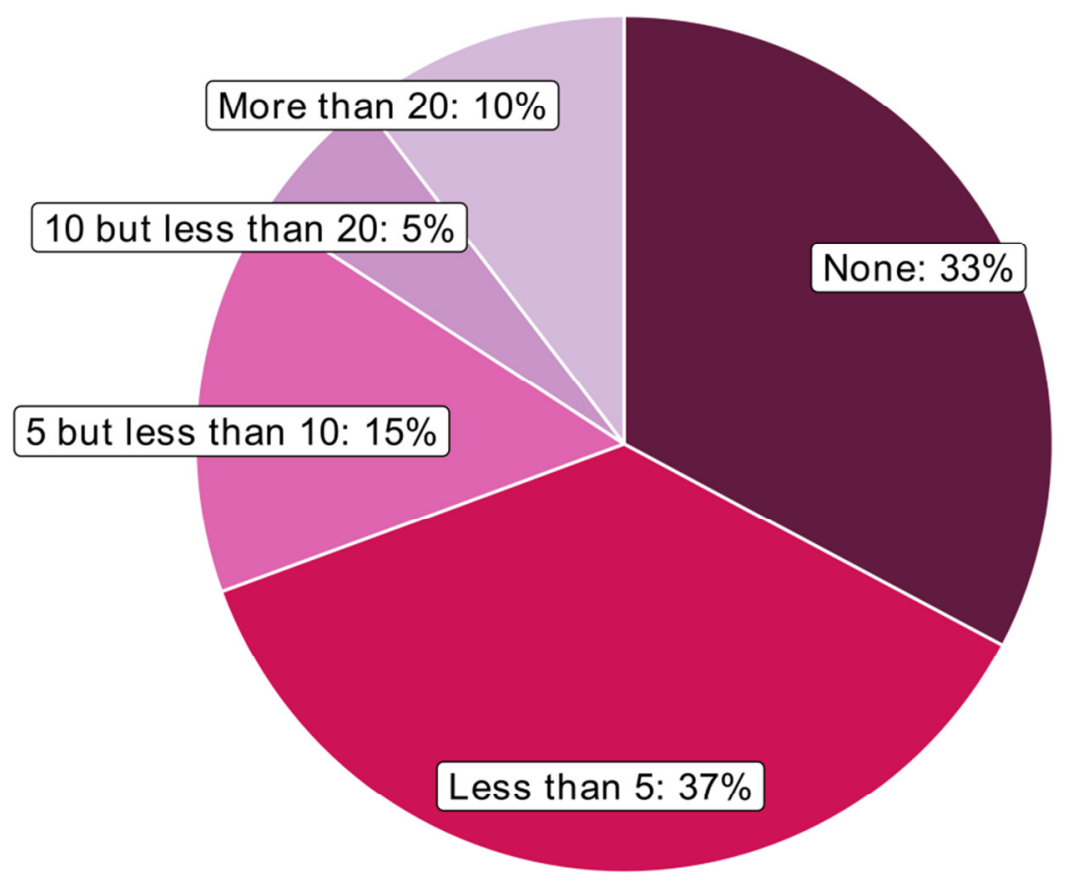

Source: City of Manchester State of the VCSE sector survey 2016/17

Base: 183

\subsection{How has the VCSE sector's workforce changed in the last 12 months?}

The survey asked respondents whether the number of staff in their organisation's workforce had 'increased', 'remained the same' or 'decreased' this year compared to the previous year. Figure 6.2 presents the results to this question, the key findings of which are: 


\section{Paid employees:}

- 53 per cent of organisations employed a similar number of paid employees compared to a year ago

- 31 per cent of organisations reported an increase in paid staff, just less than double the percentage that reported a decrease (17 per cent)

- in 2012/13, a slightly higher percentage of organisations reported an increase in paid staff (23 per cent) as did a decrease (17 per cent)

- $\quad$ across Greater Manchester 22 per cent of organisations reported an increase in their number of paid employees; while 16 per cent reported a decrease.

Figure 6.2: Change in aspects of the workforce (paid staff) in the last 12 months

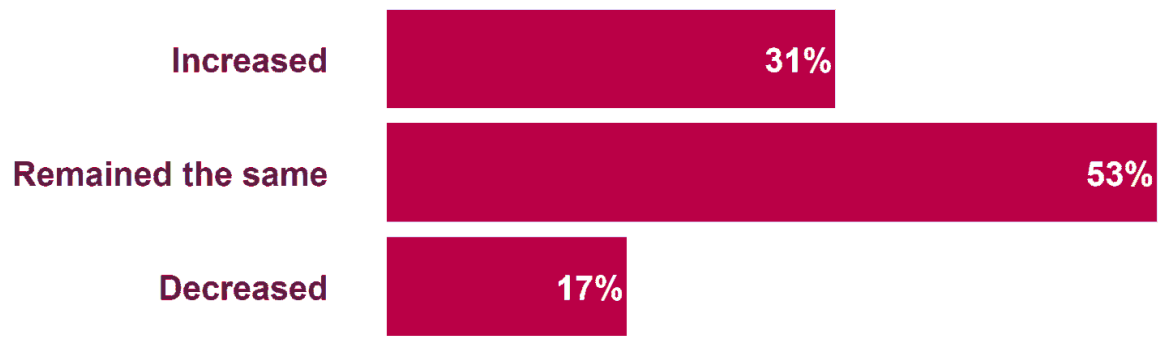

Source: City of Manchester State of the VCSE sector survey 2016/17

Base: 169

Note: 'cannot say' response has been excluded from the analysis 


\section{Volunteers}

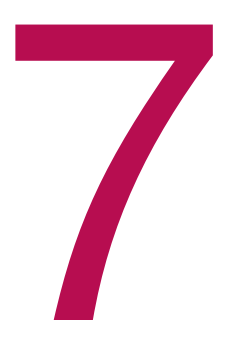

This chapter looks at the volunteers within the VCSE sector in the City of Manchester.

7.1. How many volunteers are part of the VCSE sector workforce in the City of Manchester and what is their economic contribution?

Based on responses to the survey across Greater Manchester on questions exploring the numbers of volunteers and committee/board members and the hours which they contribute, and drawing on the assumptions used to estimate the total number of organisations in the City of Manchester, it is estimated there are:

\section{0,900 volunteers or committee/board members in the VCSE sector's workforce in the City of Manchester in 2016/179}

This includes:

\section{7,400 volunteers in the VCSE sector's workforce in the City of Manchester in 2016/17}

\section{3,500 committee/board members in the VCSE sector's workforce in the City of Manchester in 2016/17}

This figure for volunteers represents 16 per cent of the City of Manchester's total population $(530,300)$ and 25 per cent of the estimated total for Greater Manchester organisations.

It is also estimated that:

\section{8,600 hours of their time provided by these volunteers and committee/board members per week}

\footnotetext{
${ }^{19}$ It is possible in cases where a person is volunteering for more than one organisation they could have been counted more than once; additionally, there will be residents from outside of the City of Manchester volunteering within the city; and conversely there will be City of Manchester residents volunteering for organisations outside of the city
} 
This represents 26 per cent of the estimated number of volunteer and committee/board member hours for all Greater Manchester organisations.

The 2012/13 study estimated there were 94,300 volunteers in the City of Manchester who provided 370,400 hours per week, both higher estimates than found for 2016/17. The previous study did not ask for volunteers and committee/board members to be recorded separately so caution should be applied when making comparisons.

There are two broad approaches to valuing the contribution of volunteers. One method, and this study's preferred approach, is to value the output that they produce. In effect this is the value to society of the goods and services that volunteers produce. This can be estimated by multiplying the number of FTE volunteers by the estimated gross value added (GVA) per FTE employee. ${ }^{20}$

From this calculation:

\section{$£ 252.1$ million per annum estimated as the economic contribution of volunteers and committee/ board members in City of Manchester organisations}

The use of estimated GVA per FTE employee to measure the value of the output produced by volunteers assumes that paid employees would not be used in the absence of volunteers to produce the same level of goods and services. In such a situation the value of output is the value of the labour input (wages and benefits) plus the value of the capital input (for example office space and computers). If paid employees would be used to produce the same level of goods and services then the value of capital input would be borne whether or not volunteers were used. Therefore the value of the output from volunteers would be just the value of the labour input. This value would be roughly equivalent to the value estimated from the input method of valuation which is outlined in the next paragraph.

In the second method, the value of the input of volunteers is used to value the contribution of volunteers ${ }^{21}$. This is the amount that it would cost to pay employees to do the work carried out by volunteers. As such, this can be considered to be the benefit to organisations ${ }^{22}$. However, this benefit might also be passed onto society via lower prices for goods and services due to lower costs of production. The input value of volunteers can be calculated by multiplying the number of hours that volunteers give per week by an estimate of how much it would cost to employ someone to do that work. There are a number of widely accepted hourly rates that could be used to estimate this value; these include: the national minimum wage or national living wage, the local median wage, the local mean wage and the reservation wage. The preference in this study has been to provide a range using the national living wage (low estimate) and the local median wage (high estimate). In reality the true value of the input provided by volunteers will lie between the two estimates. It is estimated that:

\footnotetext{
${ }^{20}$ This study used Greater Manchester GVA per employee averaged across the following two VCSE sectors: education and human health and social work activities.

21 This is the approach recommended by Volunteering England

${ }^{22}$ This assumes that there are no additional costs faced by organisations in using volunteers: for example extra management costs
} 
- $\quad$ assuming the national living wage for adults ${ }^{23}$ it would cost $£ 104.3$ million annually to employ staff to do the work provided by volunteers in City of Manchester organisations

- $\quad$ assuming the median gross hourly wage for full time employees in Greater Manchester ${ }^{24}$ it would cost $£ 186.3$ million annually to employ staff to do the work provided by volunteers in City of Manchester organisations.

Figure 7.1 presents a breakdown of responding organisations by the number of volunteers that they use. Six per cent of respondents indicated they had no volunteers, up from two per cent in the 2012/13 survey. 18 per cent had 50 or more volunteers in the latest survey, up from 14 per cent in 2012/13.

This pattern was largely representative of the picture for organisations across Greater Manchester as a whole.

Figure 7.1: Organisations by numbers of volunteers

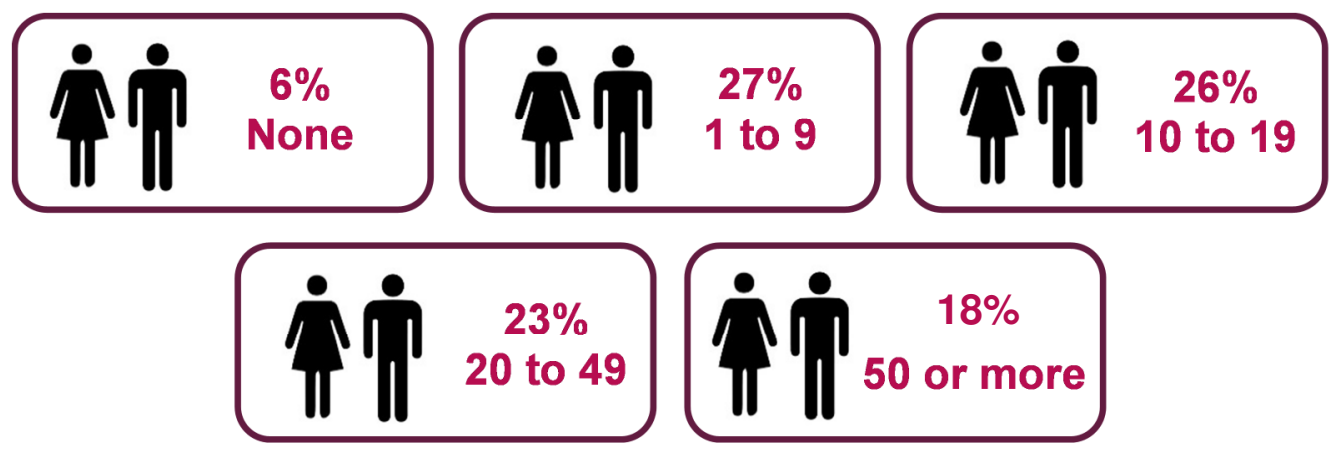

Source: City of Manchester State of the VCSE sector survey 2016/17 Base: 195

\subsection{How has the VCSE sector's workforce changed in the last 12 months?}

The survey asked respondents whether the number of volunteers in their organisation's workforce had 'increased', 'remained the same' or 'decreased' this year compared to the previous year. Figure 7.2 presents the results to this question, the key findings of which are:

- 49 per cent of respondents reported increased numbers of volunteers now compared to a year ago

- in comparison 14 per cent of organisations reported a decrease in volunteer numbers.

The 2012/13 survey found similar results, suggesting the number of volunteers within the VCSE sector has grown consistently over recent years:

- $\quad 52$ per cent of respondents reported increased volunteer numbers

- $\quad$ while 11 per cent reported that numbers of volunteers decreased.

Just over two fifths (42 per cent) of Greater Manchester organisations reported an increase in their number of volunteers over the previous year, compared with 13 per cent who reported a decrease.

\footnotetext{
${ }^{23} £ 7.20$ for 25 years and older in 2016
}

${ }^{24} £ 12.86$ for 2016 
Figure 7.2: Change in aspects of the workforce (volunteers) in the last 12 months

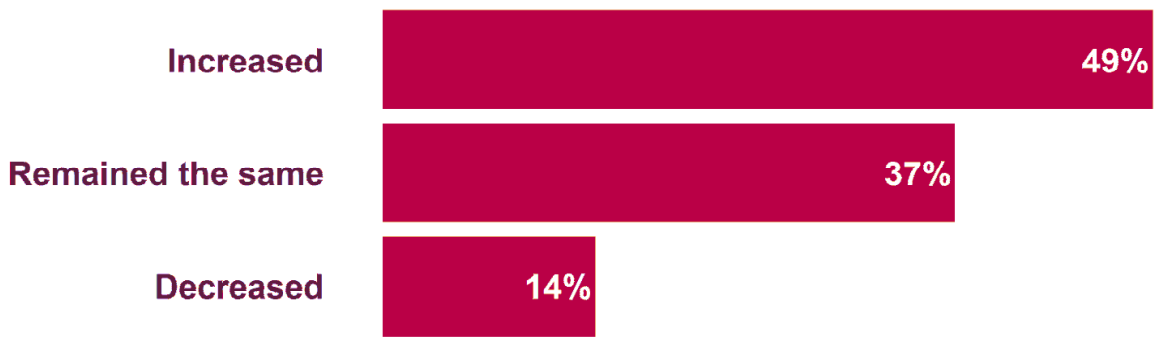

Source: City of Manchester State of the VCSE sector survey 2016/17

Base: 174

Note: 'cannot say' response has been excluded from the analysis

\subsection{Qualitative responses on volunteering}

Focus group participants from registered charities, small VCSE organisations and social enterprises were asked to discuss changes associated with volunteering in recent years. The participants from registered charities reported an increase in volunteering in recent years, mainly due to a rise in interest from young people (i.e. students). The main driver for this was the growing importance of a short 'volunteering stint' on the CV and as a stepping stone to employment.

"We have a lot more students now and we can help them focus on the jobs they want. We don't want people only motivated by philanthropy to come to work. We want people who are serious and want to gain professional experience come to work, thus raising our own professional experience. The issue is that you are going to volunteer if you want to gain professionally and we are going to gain professionally, and this has worked really well. In the past we had people not worrying too much coming to the office or being punctual, and this has changed."

However, this type of volunteer could also be problematic, as they were often not committed to the organisation(s) for the long term and sometimes had unrealistic expectations of what volunteering would entail. As a result, some VCSE organisations had struggled to properly introduce and maintain these increased numbers of volunteers. This was seen as part of a wider trend within volunteering that had been 'building' for more than a decade.

"I think volunteering has changed massively over the last 10 years because more and more people are trying to find the route to paid work through volunteering. There is this big political focus with the 'Big Society' and all and it (volunteering) is something that is mandated by a lot of university courses now I think. On the other hand, the impact of this is that now volunteers don't stay for long, they are more short term. In the past we used to have people staying for many years."

It was clear from the focus groups that many VCSE organisations have concerns about how volunteers can be used effectively and retained for the long-term whilst balancing the costs of training, managing and meaningfully involving volunteers in their work.

Focus group participants also voiced their concerns about public sector policies which interfere with their ability to effectively implement consistent volunteering programmes, especially considering service-users from vulnerable social groups (i.e., job centres' requirements, benefit sanctions, etc.). They identified the lack of state funding and support, the challenges associated with applying comprehensive training, 
monitoring and support systems for their volunteers, as the main cause of these difficulties.

"We lose a lot of people along the way, so the issue is not just to say 'use more volunteers!'. There must be the systems in place for people to be effective and for the organisation to be able to use them properly, otherwise we gain nothing from it and our target groups gain nothing from it." 


\section{Partnership Working: the Public Sector}

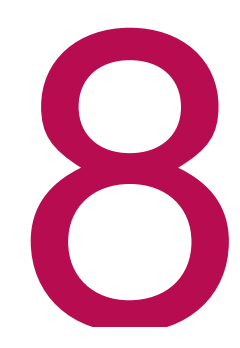

This chapter considers the relationship between the VCSE sector and the public sector, exploring organisations experiences of partnership working with Manchester City Council and other public sector bodies.

\subsection{Dealings with local public sector bodies}

Survey respondents were asked about the extent of their dealings with each of the main public sector bodies covering the City of Manchester. An overview of their responses is provided in figure 8.1, along with the local authority figure for Greater Manchester combined.

This shows that survey respondents had dealings with a range of local public sector bodies. The three most prominent were Manchester City Council, the University of Manchester and Greater Manchester Police:

- Manchester City Council: 84 per cent had some dealings with the Council; including 20 per cent who had a 'great amount' of dealings and 36 per cent who had a 'fair amount' of dealings

- University of Manchester: 61 per cent had some dealings with the University of Manchester; including five per cent who had a 'great amount' of dealings and 23 per cent who had a 'fair amount' of dealings

- Greater Manchester Police: 58 per cent had some dealings with the Police; including seven per cent who had a 'great amount' of dealings and 20 per cent who had a 'fair amount' of dealings.

Manchester City Council was also the organisation respondents had the most dealings with in the 2012/13 survey (82 per cent had some dealings) while a similar proportion in 2012/13 had dealings with the University of Manchester when compared to the latest survey (58 per cent).

Local authorities consistently emerged as the most prominent public sector contact for respondents to this study across Greater Manchester. Overall, 16 per cent of respondents said they had a 'great amount' of dealings with their local authority and 36 per cent said they had a 'fair amount'. 
Figure 8.1: Dealings with local public sector bodies

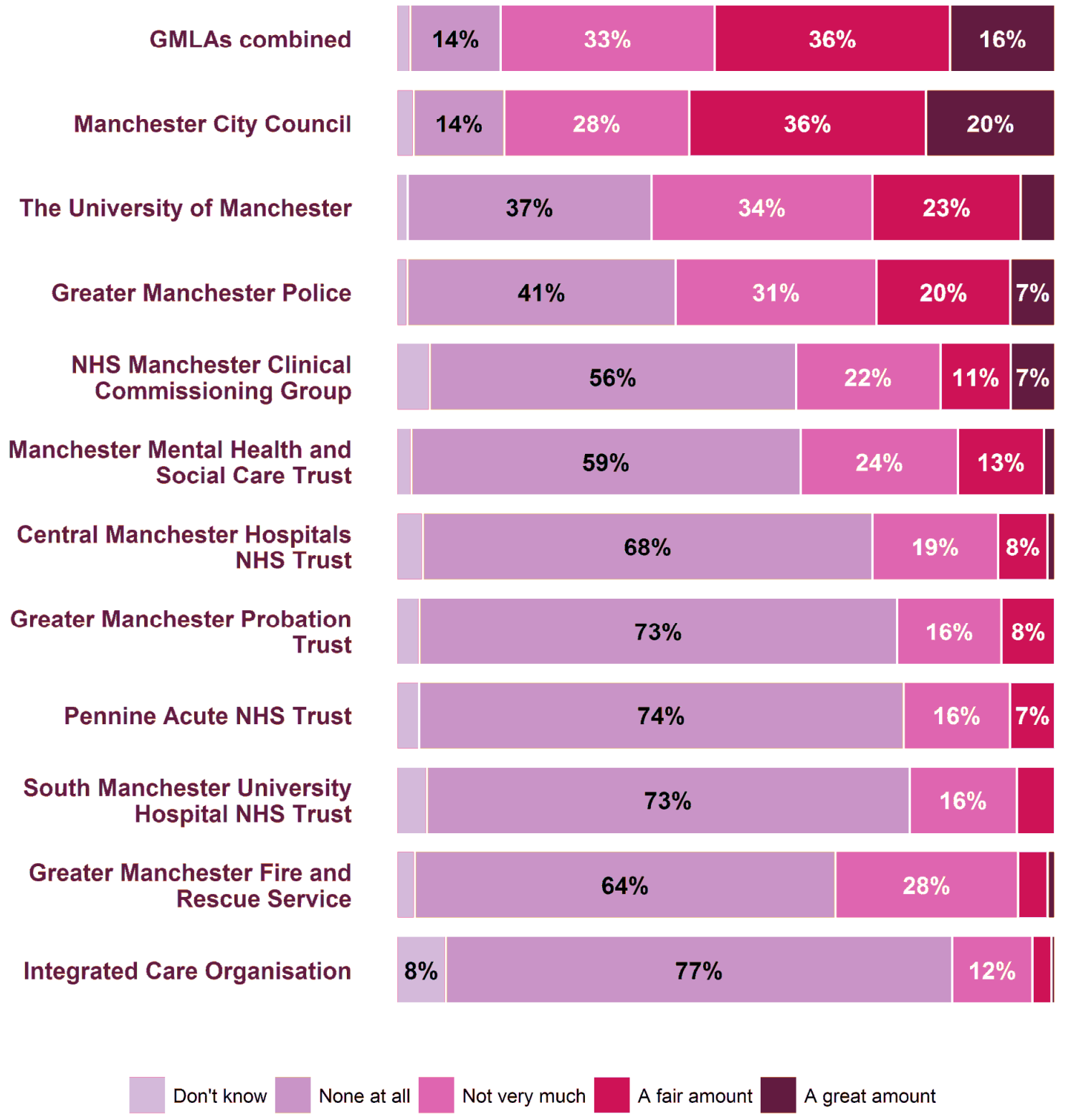

Source: City of Manchester / Greater Manchester State of the VCSE sector survey 2016/17 Base: 171-189 (City of Manchester), 1,080 (Greater Manchester)

Survey respondents were asked to indicate their most frequent public sector contact other than their local authority. Figure 8.2 shows the responses received to this question with Greater Manchester Police (27 per cent) the most commonly cited followed by the University of Manchester (24 per cent) and Manchester Health and Social Care Trust (13 per cent). 
Figure 8.2: Most frequent public sector contact other than local authority

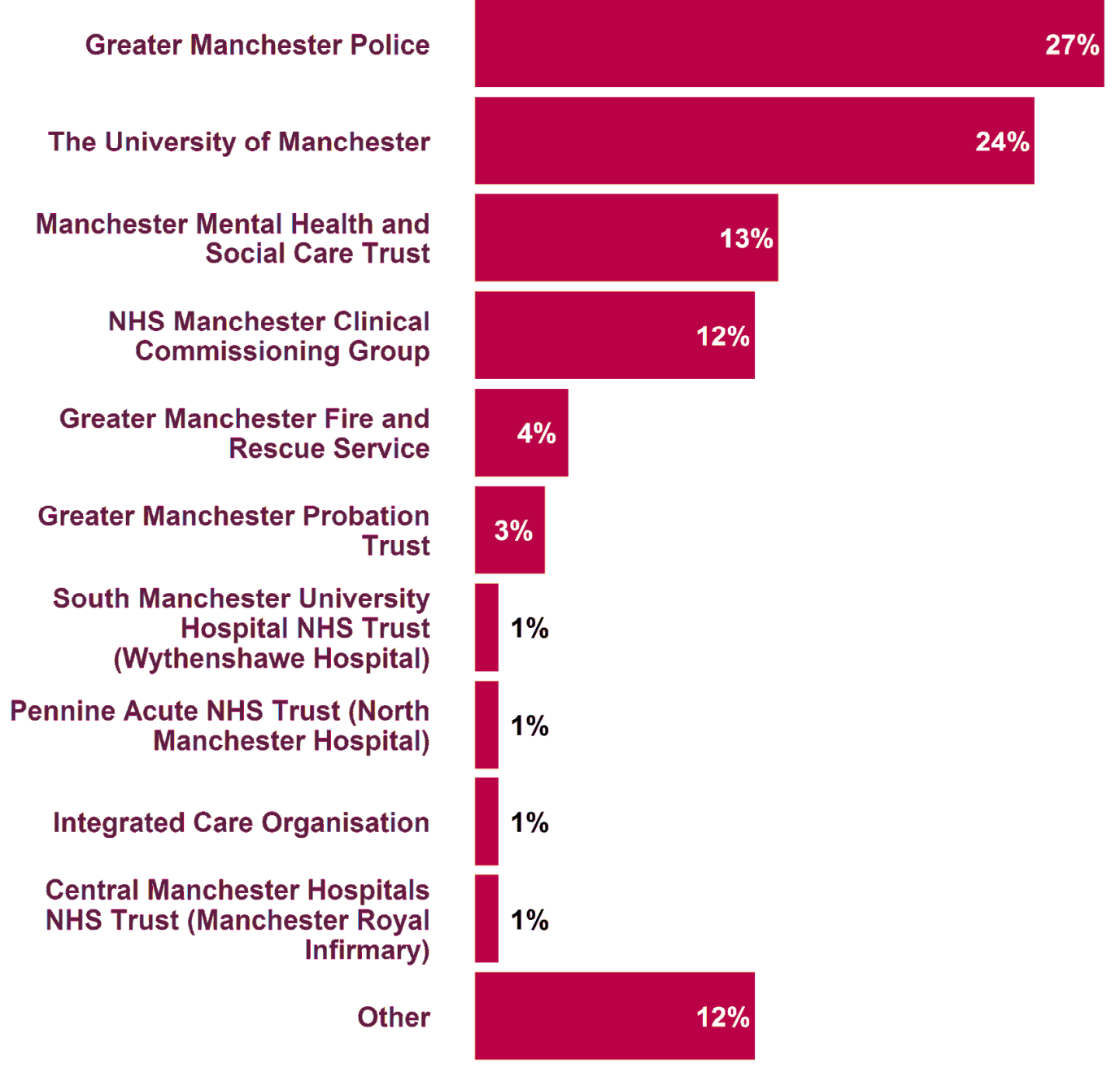

Source: City of Manchester State of the VCSE sector survey 2016/17

Base: 144

Respondents were also asked to consider the extent to which their organisation has direct dealings with any emerging Greater Manchester structures (e.g. Greater Manchester Combined Authority, The Office of the Police and Crime Commissioner, The Health and Social Care Devolution Team etc.). Figure 8.3 presents the results to this question.

Just over half ( 51 per cent) had some dealings with any of the emerging structures including just two per cent who had a 'great amount' of dealings and 16 per cent who had a 'fair amount' of dealings. Proportions were slightly lower across Greater Manchester where 38 per cent had some dealings, including two per cent who had a 'great amount' of dealings and 10 per cent who had a 'fair amount' of dealings. 
Figure 8.3: Dealings with emerging Greater Manchester structures

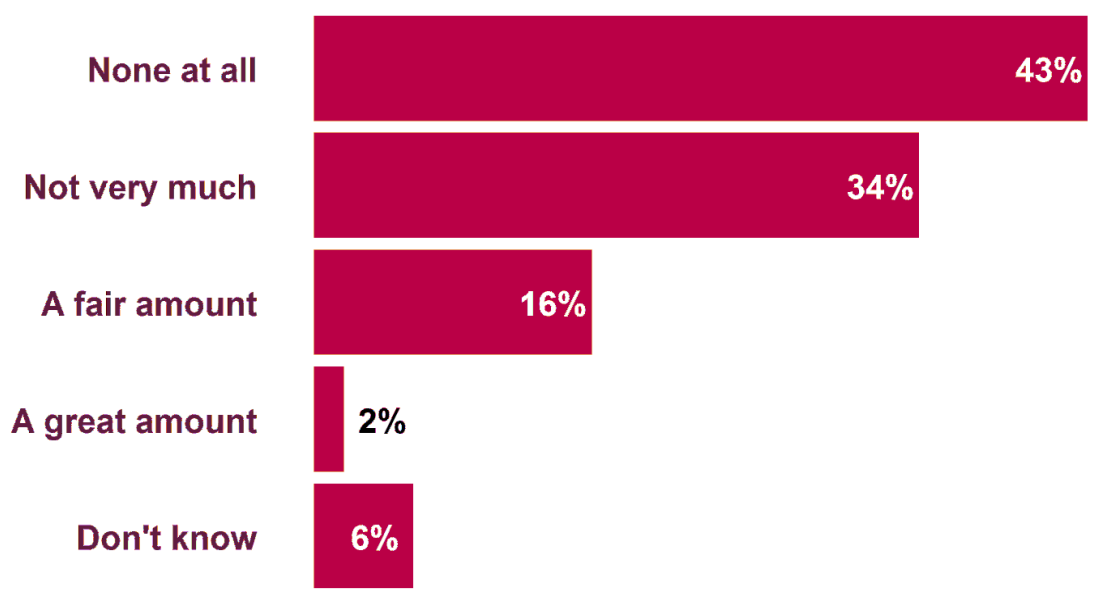

Source: City of Manchester State of the VCSE sector survey 2016/17

Base: 180

\subsection{Relationships with local public sector bodies}

Survey respondents were also asked two further questions about the extent to which their organisations were satisfied with their ability to influence public sector decisions of relevance to their organisation and the extent to which they thought local statutory bodies influenced their success ${ }^{25}$. The results of these questions are summarised in figure 8.4. A comparison with the Greater Manchester average is also provided.

Figure 8.4 shows that 25 per cent of respondents were satisfied with their ability to influence Manchester City Council decisions of relevance to their organisation and 40 per cent said that the council had a positive influence on their organisation's success. Results are similar to the Greater Manchester combined figures (30 per cent were satisfied with their ability to influence their local authority and 38 per cent agreed their local authority has a positive influence on their success). Results are slightly less positive than in 2012/13 when 30 per cent of respondents were satisfied with their ability to influence Manchester City Council decisions of relevance to their organisation and 44 per cent said that the council had a positive influence on their organisation's success.

In addition, 32 per cent of respondents said they were satisfied with their ability to influence the key decisions of their most frequent other public sector contact and 53 per cent said this contact had a positive influence on their success. These are similar to the Greater Manchester combined figures (36 per cent and 51 per cent respectively).

\footnotetext{
${ }^{25}$ This latter measure was used in 2008 and 2010 to provide evidence of local authority performance against 'National indicator 7: the environment for a thriving third sector'.
} 
Figure 8.4: Proportion of organisations who said they were satisfied with their ability to influence public sector decisions of relevance to their organisation and who said local public sector bodies influence their organisation's success

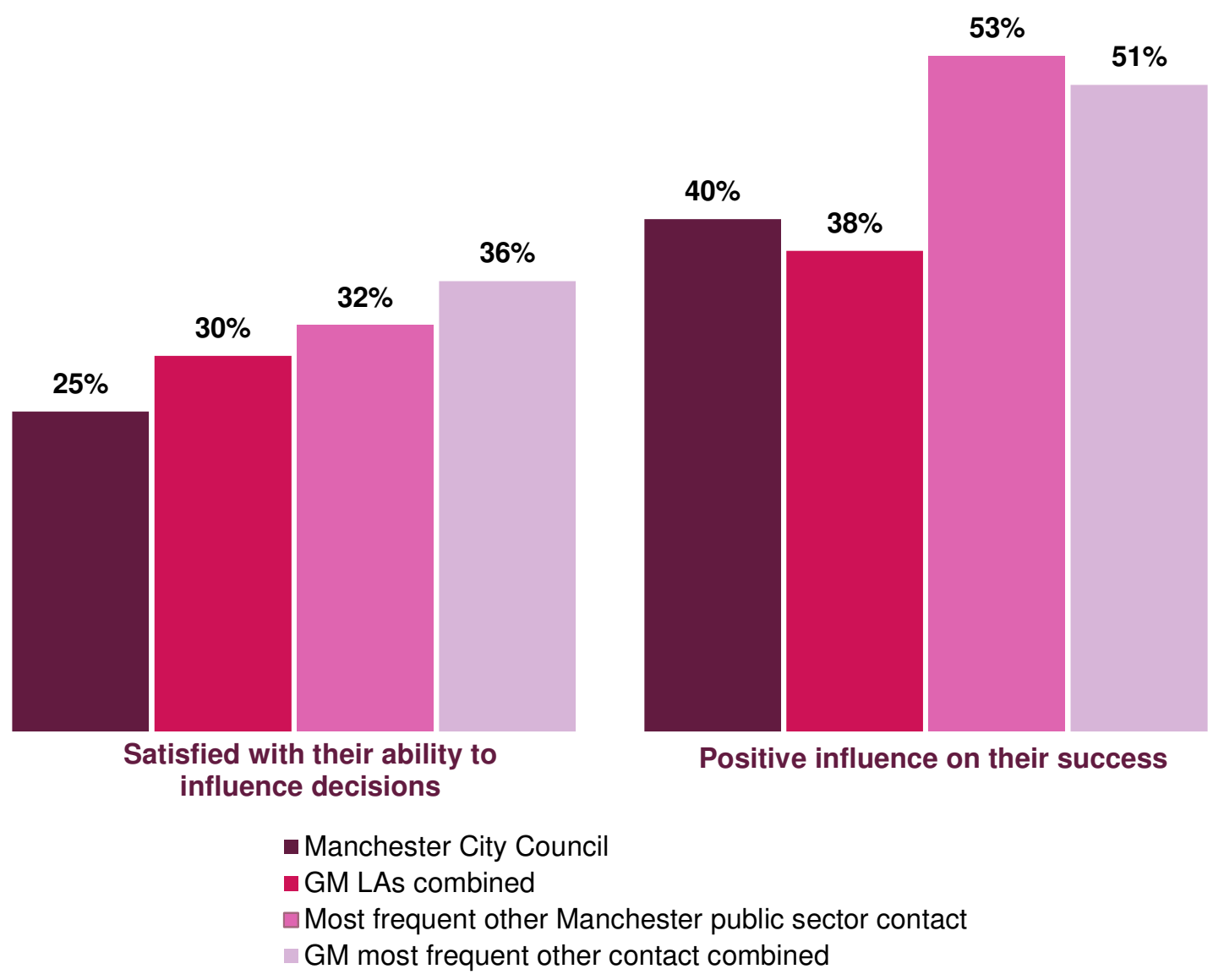

Source: City of Manchester / Greater Manchester State of the VCSE sector survey 2016/17

Base: City of Manchester: 170/109 (ability to influence), 163/118 (positive influence); Greater Manchester: 897/570 (ability to influence), 889/605 (positive influence)

\subsection{Funding from local public sector bodies}

Respondents were also asked to reflect on their experiences of public sector funding in terms of how successful they had been; how satisfied they were with bidding arrangements, and how satisfied they were with the level of opportunity to bid for long-term funding.

Figure 8.5 shows responses to the question which asked organisations to consider how successful they had been in applying for funding or bidding for contracts. Results are split between perceptions of Manchester City Council and of other public sector bodies. A comparison with the Greater Manchester average is also provided.

This shows that 51 per cent of respondents were successful in bidding for funding or contracts with Manchester City Council compared to a 34 per cent success-rate with other public sector bodies. At the Greater Manchester level, a slightly lower proportion (45 per cent) had been successful in bidding for funding or contracts from their local authority, but the figure was higher for other public sector bodies (40 per cent). 
In 2012/13 a higher proportion indicated they had been successful in bidding for funding or contracts from Manchester City Council (58 per cent) but the figure for other public sector bodies was lower (30 per cent).

Figure 8.5: Success bidding for funding and contracts

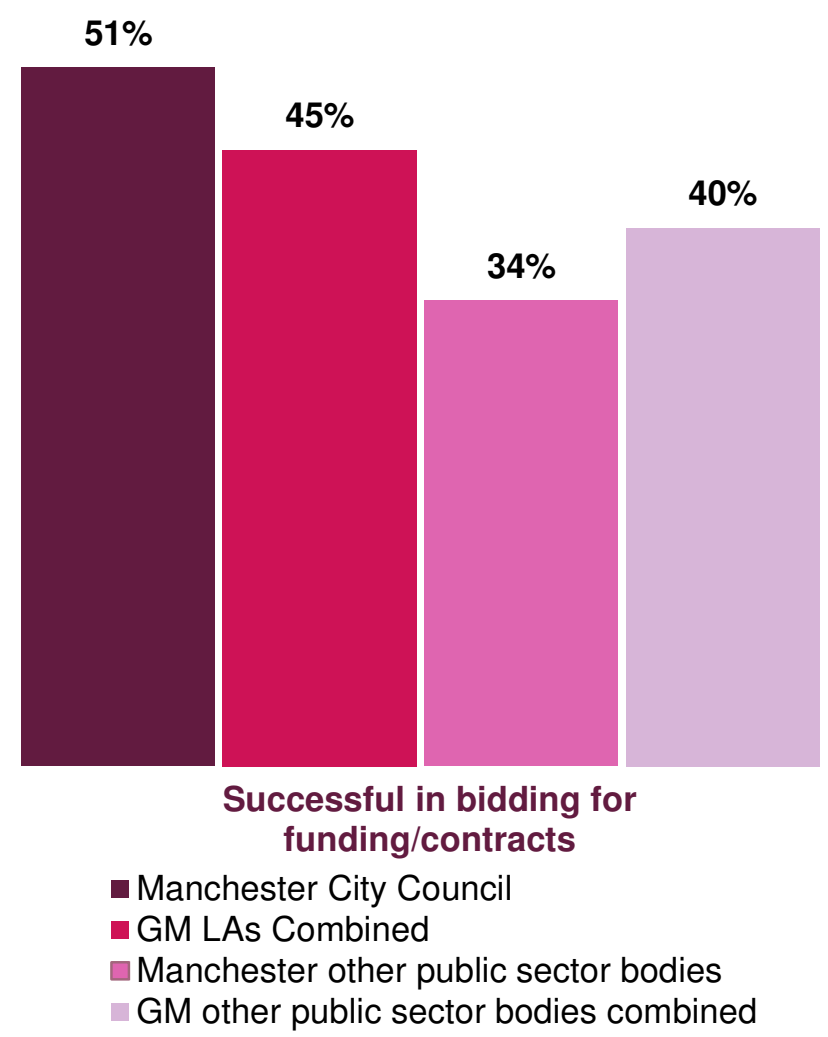

Source: City of Manchester / Greater Manchester State of the VCSE sector survey 2016/17 Base: City of Manchester: 187/181; Greater Manchester: 1,060/1,036

Respondents were asked specifically about Manchester City Council and how satisfied they were with their grant funding and contract bidding arrangements and opportunities for funding and contracts lasting three years or longer. The responses are illustrated in figure 8.6. A comparison with the Greater Manchester local authority average is also provided.

Almost two-fifths (39 per cent) were satisfied with grant funding arrangements. Satisfaction with contract bidding arrangements was lower at 26 per cent. Satisfaction with opportunities for both funding and contracts lasting three years or longer was lower again (both below 15 per cent). The pattern was similar among the Greater Manchester combined figures. In 2012/13 respondents were not asked separately about grant funding and contracts. Two-fifths ( 41 per cent) of respondents were satisfied with Manchester City Council's funding/bidding arrangements in 2012/13 and 18 per cent were satisfied with their opportunities for funding/contracts which lasted three years or longer. 
Figure 8.6: Experiences of bidding for funding and contracts with local authorities

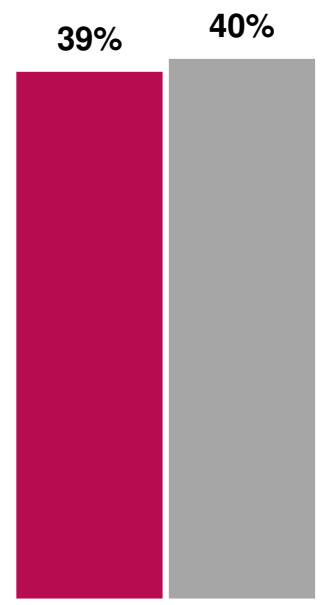

Satisfied with grant funding arrangements

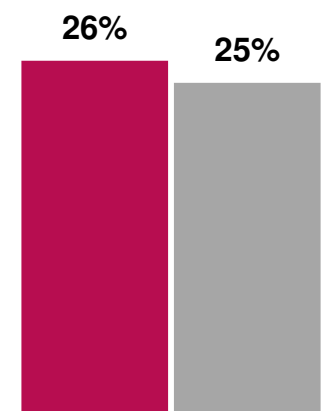

Satisfied with contract bidding arrangements

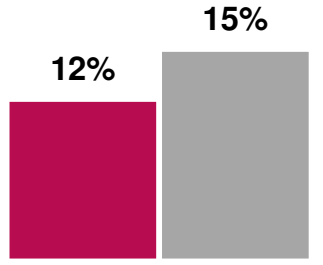

Satisfied with opportunities for

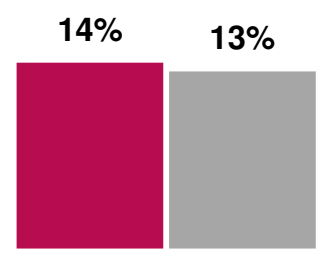

Satisfied with opportunities for funding that lasts $3 y r s+$ contracts that last 3yrs+

- Manchester City Council = GM LAs Combined

Source: City of Manchester / Greater Manchester State of the VCSE sector survey 2016/17

Base: City of Manchester: 158 (grant funding arrangements), 129 (contract bidding arrangements), 137 (opportunities for funding), 130 (opportunities for contracts); Greater Manchester: 808 (grant funding arrangements), 631 (contract bidding arrangements), 703 (opportunities for funding), 605 (opportunities for contracts)

Survey respondents were asked to consider how satisfied they were with the grant funding and contract bidding arrangements of their most frequent other public sector contact. As figure 8.7 shows, 28 per cent indicated they were satisfied, very similar to across Greater Manchester as a whole (27 per cent).

They were also asked about their satisfaction with opportunities for funding and contracts longer than three years. Just 13 per cent were satisfied, again very similar to the Greater Manchester combined figure (11 per cent). 
Figure 8.7: Experiences of bidding for funding and contracts with other public sector bodies

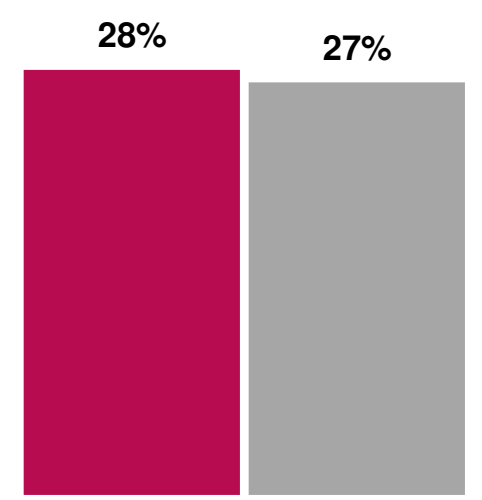

Satisfied with grant funding / contract bidding arrangements

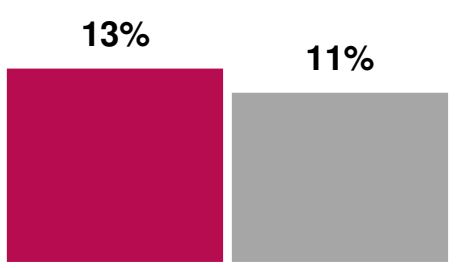

Satisfied with opportunities for funding / contracts that last 3yrs+

- Most frequent other Manchester public sector contact

- GM other most frequent contact combined

Source: City of Manchester / Greater Manchester State of the VCSE sector survey 2016/17

Base: City of Manchester: 136 (funding/bidding arrangements), 134 (opportunities for funding/contracts); Greater Manchester: 705 (funding/bidding arrangements), 687 (opportunities for funding/contracts)

\subsection{Qualitative responses on relationships between the VCSE sector and local public sector bodies}

The focus groups discussed participants' views about and experiences of working with public sector bodies in the City of Manchester. Overall, participants from all types of VCSE organisation expressed concern about a general lack of understanding of the sector amongst public sector bodies and challenges associated with opportunities to apply for funding, knowing whom to contact, and being unable to build the necessary personal relationship with key staff.

"We work with the police and a little bit with the council. But I'm not confident to send an email to someone on the council. The two have a similar language and they understand themselves very well, but they don't understand us. They are extremely capable, but they just don't know how to come down and really understand what we do."

"We used to have good relationships, but when the cuts started, they fell one after another. Too many avenues, less funding, you don't know to whom you can talk. We have less and less of a relationship over the years, especially with the CCG."

Approaches to funding, procurement and commissioning were identified as a particular issue, with a growing tendency amongst public bodies to expect tangible, wide-reaching results, without understanding or appreciating the organisations themselves, what they need and how they work; nor the real nature of actual social problems that need to be addressed. Likened to this was the concern that the public sector seemed to be increasingly focussed on short-term efficiencies and immediate, measurable outcomes; and ignored the long-term nature of the core social problems that need to be addressed. 
"They just want to see you've saved them 5,000 pounds these months. They are not interested in the long-term. So the government, the national government sends out money which is basically emergency pot money, instead of sending us work which can be more paced. You know, working with the community on long term outcomes."

"The voluntary sector really underpins a lot of people's long term futures; and it isn't that exciting to look at; it takes ages and they are not interested. It's terrible." 


\section{Partnership Working: the Private Sector}

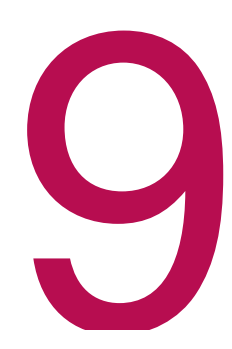

The previous chapter explored respondents' experiences of partnership working with public sector bodies. This chapter moves on to explore their experiences of working with private sector organisations. Only 27 per cent of survey respondents received any income through business donations. While this is an increase since the 2012/13 survey when just seven per cent received this type of income, this area still appears to be new territory for many VCSE organisations. Survey respondents were asked about their direct dealings and experiences of working with private businesses in the City of Manchester.

\section{1. $\quad$ Working with private businesses}

Survey respondents were asked to indicate the extent to which they had direct dealings with private businesses in the City of Manchester. Almost two-thirds (65 per cent) reported that they had some direct dealings, with 25 per cent having a 'great' or 'fair' amount of contact (figure 9.1). This is similar to the average for Greater Manchester as a whole (21 per cent 'great' or 'fair' amount of contact) and very similar to the 2012/13 survey where 67 per cent of respondents also reported some direct dealings, including 22 per cent having a 'great' or 'fair' amount of contact.

Figure 9.1: Extent of direct dealings with private businesses

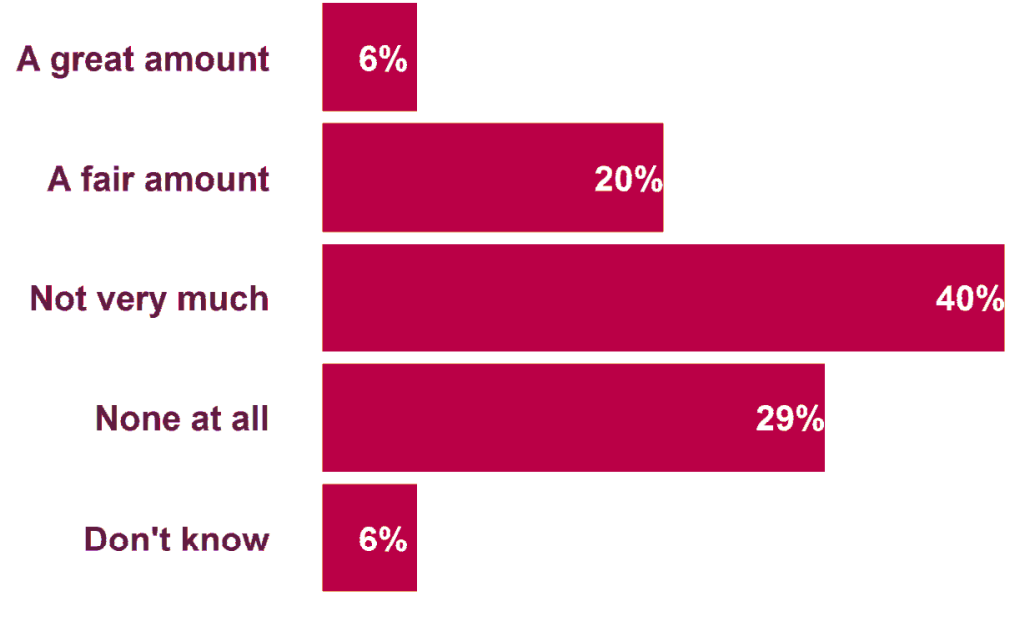

Source: City of Manchester State of the VCSE sector survey 2016/17 Base: 181 
Just three per cent of organisations indicated they are members of a formal private sector-led consortium (the same as the Greater Manchester average), while six per cent of respondents said their organisation is in formal partnership with any private sector organisations, slightly lower than the seven per cent of organisations across Greater Manchester.

Respondents were asked to comment on the influence private businesses have on their organisation's success. Taking all things into account, 27 per cent of survey respondents felt that the private business community in the City of Manchester was a positive influence on their organisation's success. This is similar to the proportion for Greater Manchester as a whole (31 per cent) and similar to the 2012/13 survey when 26 per cent of survey respondents felt that the private business community in the City of Manchester was a positive influence on their organisation's success.

Figure 9.2: Private business community's influence on VCSE sector organisations' success

Very negative influence

Negative influence

Neither positive nor negative influence

Positive influence

Very positive influence

Don't know
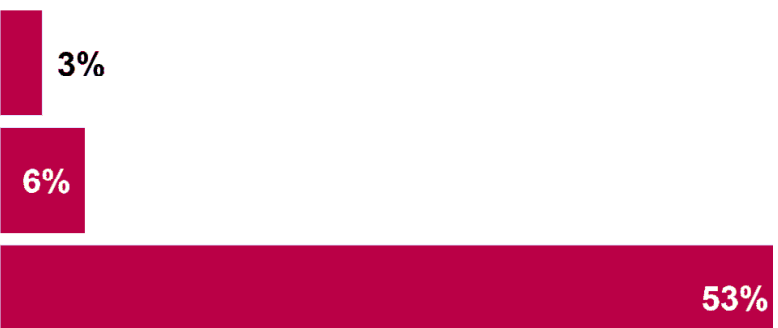

$25 \%$
$2 \%$

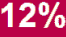

Source: City of Manchester State of the VCSE sector survey 2016/17

Base: 139

\subsection{Qualitative responses on working with private sector businesses}

Focus group participants discussed their views about and experiences of working with private businesses. Overall, participants were positive when discussing their relationship with the private sector. They indicated that partnering or just communicating with private businesses and seeing things from their perspective, can help immensely in improving the way they organise their activities, manage their resources and structure the internal governance and administrative demands of their own organisations.

"Meeting or consulting with them is very useful; we gain from this different perspective; the business perspective. There is something about having these interactions with the commercial sector and I also find working with them is often more straightforward than working with the public sector."

The feeling that working with private businesses is often simpler and more straightforward than dealing with the public sector was a consistent theme. 
"The private sector is already set. It hasn't changed so much and you find the same people throughout many years, so it's less work basically; it's simpler than public agencies. We will pay you this amount of money to do this specific piece of work."

"The public sector is so slow, things change and then this department needs authorisation from that department, etc., but with the private sector it's: 'there is your money and you go and deliver'."

When it came to building relationships with the private sector, participants suggested that this was most effective when the organisation and the business involved found 'common ground' and established trust on a personal and organisational level. Agreement that positive outcomes could be achieved through their combined resources, expertise and knowledge was also seen as a key factor in effective collaborations.

"People build partnerships, not organisations. You are a faceless organisation putting a faceless bid...it can't work that way. In the end, you get a sense if the person is appropriate and you give him this sense as well, if you are appropriate, because they will put money on the line. It can take a lot of work to establish it and maintain it, so I would say when it works, it's great with the private sector. It has been so far for us."

Where participants reported negative experiences of working with the private sector this was often because businesses in question had a limited understanding of their work and different expectations about what their relationship would or should entail.

"It has been positive I agree, but it is also difficult because I have the experience of an initially very good partnership with a business which later turned very sour, because they believed that we would keep bringing business to them and it really hadn't crossed my mind that they would have such expectations."

However, for both participants these were isolated incidents, attributed to misunderstandings resulting in a 'not so perfect fit' with the particular individuals involved, and not an indicator of a more systemic fault of the private sector which had put them off further collaboration. 


\section{Partnership Working: Voluntary Community and Social Enterprise Organisations}

The previous two chapters have explored respondents' experiences of working with organisations from the public and private sectors. This chapter discusses survey respondents' views on their work with other VCSE sector organisations.

\subsection{Working with other VCSE organisations}

Survey respondents were asked about the extent to which they had direct dealings with other VCSE sector organisations in both City of Manchester and Greater Manchester.

A large majority (92 per cent) had some direct dealings with other VCSE sector organisations in the City of Manchester, and as figure 10.1 illustrates, 76 per cent had a 'great' or 'fair amount' of contact. Results are similar, both across Greater Manchester, where 90 per cent had some direct dealings with other VCSE sector organisations in their local area and 67 per cent had a 'great' or 'fair amount' of contact, and also when compared to the 2012/13 survey, when 94 per cent had some direct dealings with other VCSE organisations in the City of Manchester and 73 per cent had a 'great' or 'fair amount' of contact.

The proportion of respondents reporting they had direct dealings with other VCSE sector organisations in Greater Manchester was lower (79 per cent), along with the proportion who had a 'great' or 'fair amount' of contact (50 per cent). Results were slightly lower at the Greater Manchester level (70 per cent direct dealings and 37 per cent with a 'great' or 'fair amount' of contact). Survey respondents were only asked about their dealings with other VCSE organisations across Greater Manchester in 2016/17. 
Figure 10.1: Extent of direct dealings with VCSE organisations

VCSE in City of Manchester

VCSE in Greater Manchester

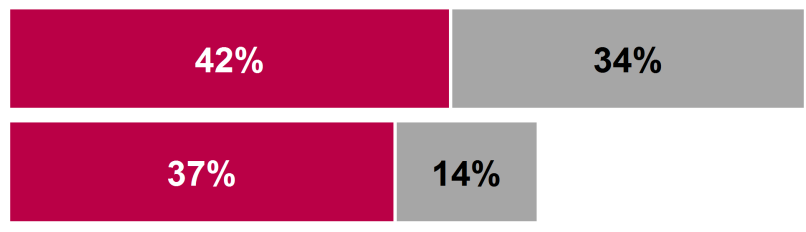

A fair amount

Source: City of Manchester State of the VCSE sector survey 2016/17

Base: 181 (City of Manchester) / 162 (Greater Manchester)

Respondents were asked to reflect on the opportunities they had to work with other VCSE sector organisations in terms of influencing local decisions, delivering local services and networking. Figure 10.2 summarises the responses.

\section{Figure 10.2: Satisfaction with opportunities to work with VCSE organisations}

$$
\begin{array}{r}
\text { Satisfied with opportunities } \\
\text { to work together to influence } \\
\text { decisions }
\end{array}
$$

$$
\begin{array}{r}
\text { Satisfied with opportunities } \\
\text { to work together to deliver } \\
\text { local services }
\end{array}
$$

Satisfied with opportunities to network with other VCSE organisations
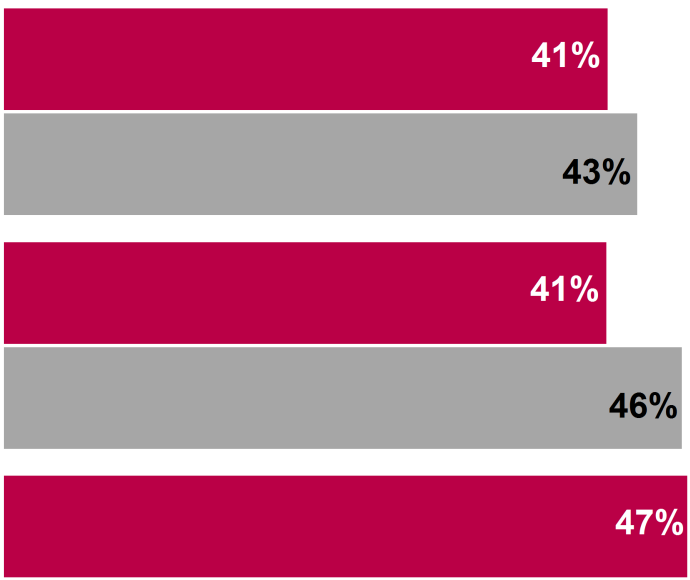

$54 \%$

Source: City of Manchester State of the VCSE sector survey 2016/17

Base: 180 (influence decisions) / 179 (delivering services) / 179 (networking)

This shows that 43 per cent of respondents were satisfied with the availability of opportunities to influence local decisions (down from 57 per cent in 2012/13) and that 46 per cent were satisfied with the availability of opportunities to work together to deliver local services (down from 52 per cent in 2012/13). A slightly lower proportion of organisations across Greater Manchester were satisfied with opportunities to influence local decisions (41 per cent), and with opportunities to work together to deliver local services (41 per cent). Over half (54 per cent) of respondents were also satisfied with opportunities to network with other VCSE organisations (47 per cent across Greater Manchester as a whole). This was a new question added to the latest survey. 
Over one quarter (26 per cent) of respondents said their organisation is a member of a formal VCSE sector consortium, slightly higher than the 22 per cent of organisations across Greater Manchester. This is clearly much higher than the two per cent in a formal private sector consortium mentioned in the previous chapter. 'GMCVO' was the most common response received when respondents were asked to specify which consortium they were a member of.

16 per cent of respondents also indicated their organisation is in another type of formal partnership with other VCSE organisations to deliver specific services (13 per cent across Greater Manchester). A wide range of responses were received when organisations were asked to specify which partnership they were members of, with a range of services covered by partnerships.

\subsection{Qualitative reflections on working with other VCSE organisations}

The focus groups discussed participants' views about and experiences of working in partnership with other VCSE organisations in the City of Manchester and more widely. Overall, these experiences have been positive, with participants highlighting the importance of a 'good fit' between organisations in terms of activities, goals, experience, knowledge and identity.

"Yes, this is something I feel very strongly about and I think we do need to join forces with like-minded organisations. We need to be united to move forward."

"I think that there needs to be something that binds us and unites us formally. It's so fundamental to work beneath the same umbrella and be this huge force for positive change, but I don't see this happening as much as I would like."

Participants highlighted the importance of building personal relationships with colleagues in other VCSE organisations: once they had worked with someone and had gained trust good long term relationships could be developed.

"It's you know, once you do something and it goes well and build relationships with people, there is really no reason not to team-up when there is an opportunity. I don't think there is any real competition between us."

"Like others said, when you determine what is a good fit, we usually proceed nicely. If there are problems with a partnership, it simply doesn't materialise or it won't in any future occasions. It depends on the specifics mostly."

A number of participants indicated that they would be hesitant about working with some of the larger more business-oriented VCSE organisations. They expressed concern that some of these organisations are specifically 'geared' towards competing and earning the 'big funding bids' and that their values and ways of doing things could be at odds with the identity of the smaller more values-led VCSE organisations. 


\section{The Future}

This chapter details the responses received to questions about the future in the survey of organisations.

\subsection{Factors assisting or constraining delivery}

Respondents were asked to consider the factors they anticipated assisting or constraining their organisation over the next 12 months. Figure 11.1 illustrates that over half of respondents thought the following factors would assist their organisation over the next year:

- ability to secure other sources of income: 54 per cent anticipated this factor assisting their organisation; including 23 per cent who saw this as 'greatly assisting' and 31 per cent 'assisting'

- engagement with other VCSE organisations: 52 per cent anticipated this factor assisting their organisation; including 10 per cent who saw this as 'greatly assisting' and 41 per cent 'assisting'.

Engagement with other VCSE organisations was the most common factor selected across Greater Manchester, with 50 per cent of organisations envisaging this factor assisting their organisation over the next 12 months. Ability to secure other sources of income was rated only slightly less highly, with 47 per cent envisaging this factor as assisting their organisation over the same period.

In contrast over 30 per cent saw the following factors as constraining their organisation over the next 12 months:

- the local economy: 36 per cent anticipated this factor constraining their organisation; including 12 per cent who saw this as 'seriously constraining' and 23 per cent 'constraining'

- ability to secure grant funding from public sector bodies: 32 per cent anticipated this factor constraining their organisation; including 14 per cent 'seriously constraining' and 18 per cent 'constraining'

- $\quad$ ability to employ staff with sufficient skills: 31 per cent anticipated this factor constraining their organisation; including 13 per cent who saw it as 'seriously constraining', and 17 per cent 'constraining'.

The local economy was also the most common factor selected across Greater Manchester as a whole, with 38 per cent anticipating this factor constraining their organisation over the following year. 
Figure 11.1: Factors anticipated as assisting or constraining organisations over the next 12 months

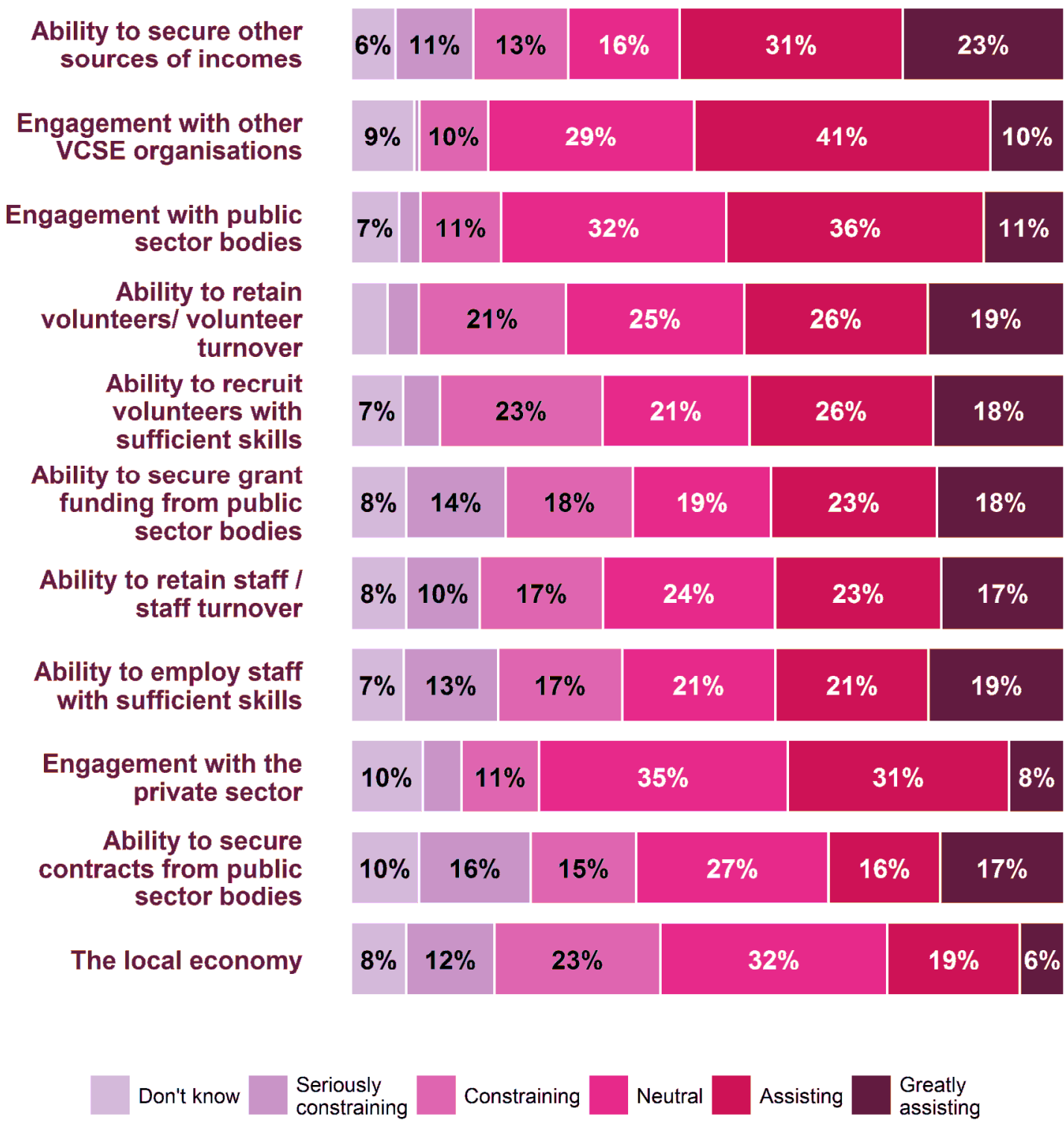

Source: Manchester City State of the VCSE sector survey 2016/17

Base: 115-136

Following on from quantitative questions regarding the factors organisations anticipated assisting or constraining their organisation over the next year, respondents were also asked to provide further qualitative (i.e. written) information about these factors.

Perhaps unsurprisingly common themes included the potential impact of ongoing public sector austerity and an associated increase in demand for services:

"As always, the challenge is short term funding combined with high levels of expectation/ demand. We have greatly increased our ability to generate income but the marketplace continues to shrink and there is, particularly with public sector partners, a continued resistance to paying us for our time, skills and knowledge" 
"With the cuts to local authorities, along with the furthering of austerity policies, I think there is likely to be an increase of pressure on charitable and voluntary organisations to pick up the slack with little or no additional funding"

"As the direct result of service gaps/losses and cuts in government funding over this past year we have had increased users outstripping supply"

"a backdrop where I feel the local economy is going to face some serious financial crisis, as a result of continuing government cuts to local authorities, continued austerity and the new challenge of Brexit"

"The main challenge for our organisation is meeting demand. Due to cuts in services and closure of other provisions, we have seen a fourfold increase in the number of enquiries and people registering with us for support"

"The inevitable cuts to public funding is a serious threat to all VCSE organisations and this means we will sink under the pressures of less funding"

Finding, retaining and paying for appropriately skilled staff was also identified as a priority by a number of organisations:

"Finding staff with the right skills is an ongoing battle"

"Not having sufficient budgets to remunerate staff properly or provide incentives mean we lose staff or have less ability to offer training"

\subsection{Current and future strategies}

Survey respondents were asked what strategies they are actively pursuing or planning to pursue. Figure 11.2 summarises the responses received and shows that around seven out of ten or more respondents were already doing or planning to do the following:

- working more closely with another voluntary/not-for-profit organisation: 79 per cent were already pursuing or planning to pursue this strategy; including 35 per cent who are doing this now and 44 per cent who are planning to do this in the future

- $\quad$ increasing earned income: 71 per cent were already pursuing or planning to pursue this strategy; including 34 per cent who are doing this now and 37 per cent who are planning to do this

- changing the way you run your services or activities: 69 per cent were already pursuing or planning to pursue this strategy; including 26 per cent who are doing this now and 43 per cent who are planning to do this.

Results across Greater Manchester followed the same pattern, with the same ordering of strategies being pursued or planning to be pursued. 
Figure 11.2: Strategies being planned or pursued

Working more closely with
another voluntary/not-for-
profit organisation

Increasing earned income

Changing the way you run your services or activities

Working more closely with a public sector organisation

Increasing individual donations

Working more closely with a private sector company

Merging with one or more similar organisations

Borrowing money to increase the volume of activity/enter new areas of work
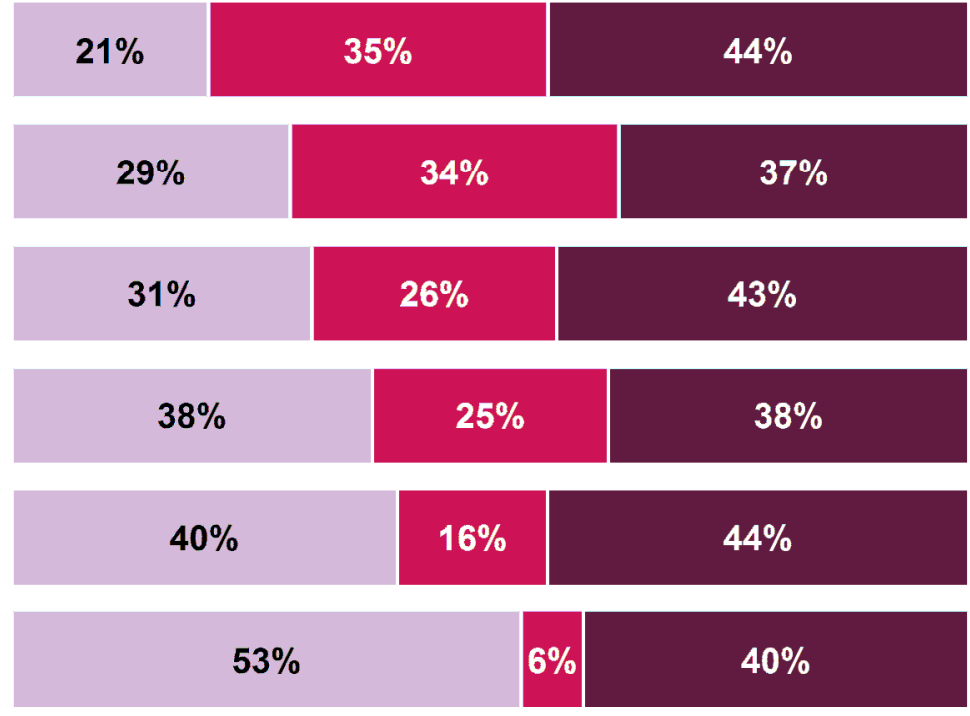

$81 \%$ $7 \% \quad 13 \%$

\section{$90 \%$}

Not doing this now and not planning to do this

Doing this now

Planning to do this

Source: City of Manchester State of the VCSE sector survey 2016/17

Base: 129-141 


\section{Conclusions}

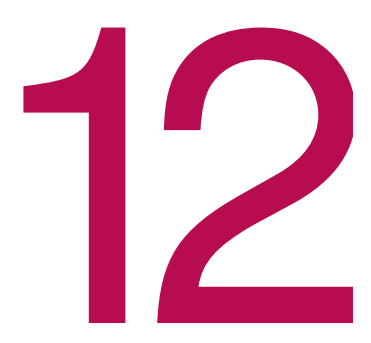

1. The VCSE sector in the City of Manchester continues to occupy an important strategic position between policy development, service provision and everyday life.

There are an estimated 3,394 organisations working in the VCSE sector in Manchester who are involved in many areas of activity.

As in the 2013 study, the local area and specific communities and neighbourhoods within it are a main focus for the majority of organisations; 32 per cent identified particular neighbourhoods or communities in Manchester as their highest main geographic focus.

The thematic areas with the greatest proportion of organisations working in them are: community development; health and wellbeing; and education, training and research; the same three areas selected most frequently in 2012/13.

The VCSE sector plays a key role in fostering strong and cohesive communities within the City of Manchester and is an essential part of the social fabric of the City. About three-quarters (74 per cent) of organisations felt they are addressing the needs of disadvantaged members of the community. Other common responses were improving people's mental wellbeing (66 per cent), increasing people's skills (66 per cent) and encouraging people to be involved in regular volunteering (65 per cent).

2. The sector in the City of Manchester remains an important economic player, contributing significantly to $\mathrm{GVA}^{26}$, but patterns in income, expenditure and the level of reserves suggest that, as in 2013, the sustainability of many organisations may be under threat.

Total income of the VCSE sector in $2014 / 15$ is estimated to be $£ 526$ million. This represents an increase of three per cent compared to 2013/14 when the total income of the VCSE sector was an estimated $£ 509$ million. The vast majority of organisations are micro or small although the majority of income is concentrated in large and medium-sized organisations.

The picture is more positive overall than in the previous 2013 study which identified year-on-year reductions in income. However analysis of income data across Greater Manchester by organisation size revealed micro and small organisations experienced year on year reductions in total income between 2012/13 and 2014/15. By contrast medium and large organisations saw a reduction in total income between 2012/13 and 2013/14 but then an increase between 2013/14 and 2014/15.

\footnotetext{
${ }^{26}$ Gross Value Added (GVA), the value of goods and services produced, is a key measure of the economic contribution of organisations or sectors.
} 
Over half (57 per cent) of respondents reported increasing their expenditure but only 47 per cent had experienced an increase in income and only 38 per cent report an increase in reserves.

In addition, 31 per cent of respondents reported a decrease in income but only 18 per cent reduced their expenditure.

20 per cent of respondents provided an expenditure figure for 2014/15 that was greater than their income.

These results indicate a sizeable number of organisations spent more money than they received in the last 12 months and that a considerable number of organisations are using their reserves to supplement their income, potentially leaving them in a fragile financial position.

\section{The VCSE sector in the City of Manchester continues to provide significant social value.}

It is estimated that the VCSE sector in Manchester made 6.1 million interventions with clients, users or beneficiaries in the previous year.

VCSE organisations work with a range of different people, especially children and young people and black and minority ethnic communities, but also people from vulnerable groups (for example those with health problems or unemployed people).

\section{The VCSE sector continues to be a significant employer.}

In 2016/17 there were an estimated 11,000 FTE paid staff. In addition the sector was supported by 87,400 volunteers and 23,500 committee/board members who combined donated 278,600 hours per week.

Valuing the contribution of paid employees to City of Manchester organisations by the expected value of the output that they produced gives an estimated annual contribution of $£ 347$ million. Doing the same for volunteers and committee/board members gives an estimated contribution of £252.1 million.

5. The number of volunteers within the sector in the City of Manchester appears to have grown consistently over recent years, however there are challenges associated with volunteering across the City.

Almost half of organisations responding to the survey reported increased numbers of volunteers compared to the previous year, while just 14 per cent of organisations reported a decrease in volunteer numbers.

Focus group participants from registered charities reported an increase in volunteering in recent years, mainly due to a rise in interest from young people (i.e. students). The main driver for this was the growing importance of a short 'volunteering stint' on the CV and as a stepping stone to employment. However, this type of volunteer could also be problematic.

Many VCSE organisations have concerns about how volunteers can be used effectively and retained for the long-term whilst balancing the costs of training, managing and meaningfully involving volunteers in their work. 


\section{There is a mixed picture in the City of Manchester regarding relationships between the VCSE sector and public sector bodies.}

Overall, 84 per cent of respondents in Manchester had some dealings with Manchester City Council (82 per cent in 2012/13): 20 per cent had a great amount of dealings with the Council and 36 per cent had a fair amount of dealings.

One quarter of respondents were satisfied with their ability to influence Manchester City Council decisions of relevance to their organisation while 40 per cent said Manchester City Council had a positive influence on their organisation's success. Results are slightly less positive than in 2013.

Overall, focus group participants from all types of VCSE organisation expressed concern about a general lack of understanding of the VCSE sector amongst public sector bodies and challenges associated with opportunities to apply for funding, knowing whom to contact, and being unable to build the necessary personal relationship with key staff.

Approaches to funding, procurement and commissioning were identified as particular issues as was concern that the public sector seemed to be increasingly focussed on short-term efficiencies and immediate, measurable outcomes; and ignored the longterm nature of the core social problems that need to be addressed.

\section{Engagement with private businesses remains relatively low and perceptions of the private business sector appear to have remained stable.}

65 per cent of organisations had some direct dealings with private businesses, with 25 per cent having a 'great' or 'fair' amount of contact. This is similar to 2012/13 when 67 per cent reported some direct dealings, including 22 per cent having a 'great' or 'fair' amount of contact.

Over one quarter (27 per cent) felt that the private business community in the City of Manchester was a positive influence on their organisation's success. This is similar to $2012 / 13$ when 26 per cent agreed private businesses were a positive influence.

Overall, focus group participants were positive when discussing their relationship with the private sector. They indicated that partnering or just communicating with private businesses and seeing things from their perspective, can help immensely in improving the way the organise their activities, manage their resources and structure the internal governance and administrative demands of their own organisations.

The feeling that working with private businesses is often simpler and more straightforward than dealing with the public sector was a consistent theme.

Where focus group participants reported negative experiences of working with the private sector this was often because businesses in question had a limited understanding of their work and different expectations about what their relationship would or should entail.

\section{The VCSE sector in the City of Manchester continues to be well connected internally although most contact appears to be informal.}

As in the 2013 study, the vast majority of organisations had some direct dealings with other VCSE sector organisations in their local area, including 76 per cent who had a 'great' or 'fair amount' of contact.

26 per cent of respondents said their organisation is a member of a formal VCSE sector consortium. 
Overall, focus group participants were positive about working closely with other organisations in the sector, with participants highlighting the importance of a 'good fit' between organisations in terms of activities, goals, experience, knowledge and identity.

A number of participants indicated that they would be hesitant about working with some of the larger more business-oriented VCSE organisations, expressing concern that some of these organisations are specifically 'geared' towards competing and earning the 'big funding bids' and that their values and ways of doing things could be at odds with the identity of the smaller more values-led VCSE organisations.

\section{The sector still faces an uncertain future.}

With austerity measures set to continue for the foreseeable future and public sector funding for the sector continuing to be squeezed, there are still reasons for caution within the sector.

Respondents appear to recognise this uncertainty and are pursuing a range of strategies to ensure their sustainability, in particular: generating earned income from other sources, partnership working and organisational change. 


\section{Appendix 1}

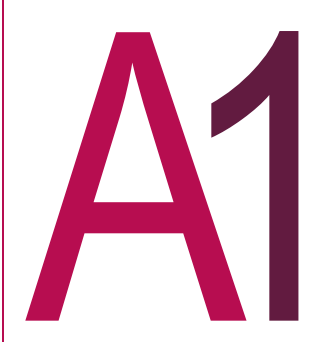

\section{Methodology}

\section{Survey of organisations}

At least partial responses were received from 63 of the 1328 organisations that were sent a survey questionnaire: this represents a response rate of five per cent. Another web-based version of the survey was also distributed by Macc, reaching organisations also included in the original sample and beyond. In addition GMCVO distributed a version of the survey via their networks. A further 138 responses were collected via this method, meaning a total of 201 responses were collected overall during September 2016 - January 2017, giving a higher overall response rate.

The survey was undertaken as part of a wider study in six other Greater Manchester boroughs: Bolton, Salford, Oldham, Rochdale, Stockport and Tameside.

The questionnaire was based on the one originally developed for the 'State of the Voluntary Sector Survey' undertaken in Salford in 2010. The questionnaire was revised for the 'Greater Manchester State of the Voluntary Sector' research undertaken in 2012/13 and again for this wave of the survey following input from the Research Steering Group. The Greater Manchester Chief Officers Group also provided additional oversight regarding the survey design and implementation.

The questionnaire provided data on various aspects of the VCSE sector including:

- the scale and scope of its activity, including the roles organisations undertake, the people they support, and the areas they benefit

- the economic impact of its work, including income and expenditure, sources of funding, the role of paid staff and volunteers, and financial sustainability

- relationships with the public sector, including Manchester City Council, public sector health bodies, and a range of other local statutory bodies

- relationships with other local organisations, including VCSE organisations and private businesses.

Where possible the report compares results from the latest survey and the 2012/13 study. Revisions to the questionnaire mean that comparisons are not always possible or appropriate. It is also worth noting that in 2012/13 a large postal survey was the main method of data collection which was supplemented with a web based survey. This is different to the latest study when a web based survey was the primary method of data collection.

When reading the report it is important to acknowledge two key points. First, the results reported are based on the survey responses received. Therefore it is possible that if a different sample of organisations had taken part in the survey different results may have emerged. It is estimated that the results reported are within $+/-6.7$ percentage points of the true value. 
Secondly, on a number of occasions the analysis in this report has used extrapolations from the survey responses to provide estimates of totals for all organisations that work in the VCSE sector including:

- the number of clients, users and beneficiaries of the sector

- the total income of the sector

- $\quad$ and the number of FTE paid staff and the number of volunteers and committee/board members that are part of the sector's workforce; including the hours per week that volunteers contribute.

In each case the same three stage method has been used for calculating the sector wide totals:

- $\quad$ stage one: calculate the Greater Manchester averages for each of the four size bands of organisations: 'micro', 'small', 'medium' and 'large': column (a) in table A1

- $\quad$ stage two: multiply the average for each size band (column (a) in table A1) by the estimated number of organisations within that size band (column (b) in table A1) to give the total for each size band of organisations (column (c) in table A1)

- $\quad$ stage three: sum the estimates from stage two (column (c) in table A1) to give a sector wide total estimate (cell (d) in table A1).

This was necessary to take account of noticeable differences in the response rates by organisation size. A failure to do this would lead to upwardly biased estimates: a small number of mainly 'large' organisations create a high mean value that is not representative of the majority of organisations. This is an important point given that we estimate that a large proportion of the sector is made up of 'micro' organisations which tend to have far lower values and not taking into account difference by size of organisations would produce estimates that are much higher.

Table A1: Extrapolations: a worked example (total annual income)

\begin{tabular}{lrrr}
\hline & $\begin{array}{c}\text { Average income by } \\
\text { size } \\
\text { (a) }\end{array}$ & $\begin{array}{c}\text { Estimated number } \\
\text { of organisations } \\
\text { (b) }\end{array}$ & $\begin{array}{c}\text { Total income } \\
\text { (thousands) } \\
\text { (c) }\end{array}$ \\
\hline Micro (under $£ 10 k)$ & $£ 2,438$ & 2,247 & $£ 5,476,388$ \\
Small (£10k to £100k) & $£ 38,844$ & 513 & $£ 19,909,889$ \\
Medium (£100k to £1m) & $£ 320,581$ & 477 & $£ 152,860,213$ \\
Large (over £1m) & $£ 2,201,023$ & 158 & $£ 347,976,313$ \\
\hline Total & & & (b) $£ 526,222,802$ \\
\hline
\end{tabular}

Please note it has been assumed here that the estimated averages for Greater Manchester organisations are representative for organisations within the City of Manchester. So for example it is has been assumed that the estimated average income of approximately $£ 320,600$ for medium sized organisations across Greater Manchester is representative of the income for medium sized organisations within the City of Manchester.

Using the Greater Manchester averages improves the reliability of the estimates.

\section{Focus groups}

To provide a further depth of understanding to some of the themes covered in the State of the Sector Survey two focus groups were conducted. The groups were held midway through 
the survey administration and undertaken by Macc who recruited local organisations to participate in the groups.

A topic guide was devised to help guide discussions and ensure a standardised approach across all local authority areas conducting focus groups. The topic guide was created in partnership between CRESR and the Research Steering Group with CRESR providing advice and guidance on best practice in undertaking this type of research.

The focus groups lasted approximately 1 hour - 1 hour 30 minutes and were digitally recorded where consent was obtained from all participants. The recordings were then provided to CRESR who analysed the discussions. Analysis of the discussions is included in the relevant chapters of this report.

The topics discussed in the focus groups concentrated on four key themes: volunteering, working with the public sector, working with other VCSE organisations and working with the private business sector.

One focus group took place with small VCSE groups/social enterprises and the other with registered charities.

\section{Legal status of responding organisations}

Respondents to the questionnaire were asked to identify the legal status of their organisation. For this question it was possible for organisations to select registered charity in addition to identifying their legal form. Figure A1 below shows that 20 per cent were a group with a constitution, but not registered charities, 39 per cent of organisations were a company limited by guarantee and that separate to identifying their legal status the majority of respondents, 57 per cent, identified that their organisation was a registered charity.

These results are largely similar to those in the $2012 / 13$ survey when:

- 25 per cent of organisations responding to the survey were a group with a constitution, but not registered charities

- 29 per cent were companies limited by guarantee

- five per cent of organisations had no legally constituted form

- 56 per cent of respondents identified that their organisation was a registered charity.

Across Greater Manchester:

- 30 per cent of organisations responding to the survey were a group with a constitution, but not a registered charity

- 28 per cent were a company limited by guarantee

- four per cent of organisations had no legally constituted form

- 49 per cent of organisations were registered charities. 
Figure A1: The legal status of responding organisations

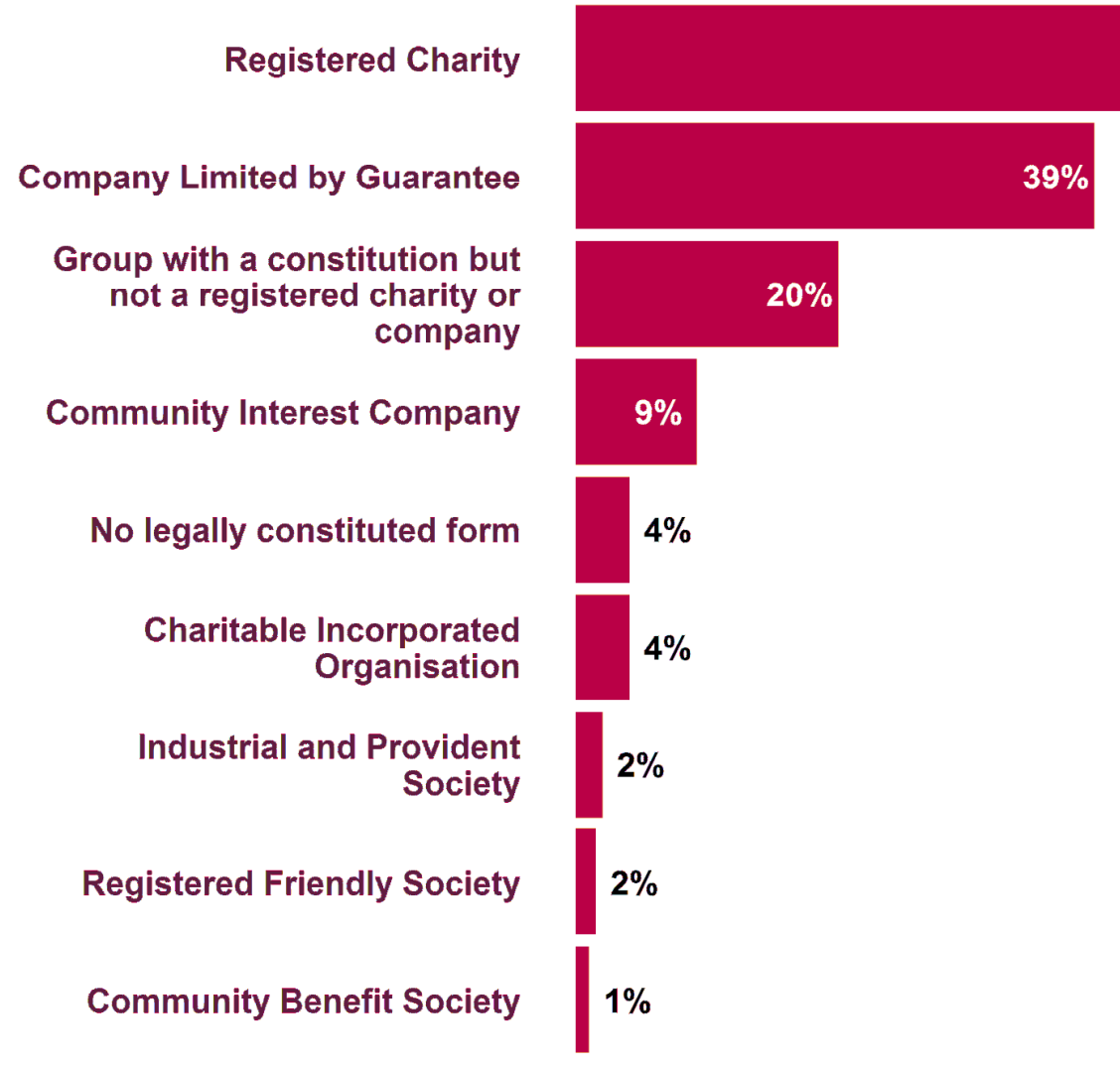

Source: City of Manchester State of the VCSE sector survey 2016/17

Base: 199 


\section{Sheffield Hallam University}

City of Manchester state of the voluntary, community and social enterprise sector 2017 : A report on the social and economic impact

DAMM, Christopher <http://orcid.org/0000-0002-7355-3496>, PRINOS, loannis and SANDERSON, Elizabeth <http://orcid.org/0000-0003-1423-1670>

Available from the Sheffield Hallam University Research Archive (SHURA) at:

http://shura.shu.ac.uk/16668/

\section{Copyright and re-use policy}

Please visit http://shura.shu.ac.uk/16668/ and http://shura.shu.ac.uk/information.html for further details about copyright and re-use permissions. 